ANALYSIS OF THE VIBRATIONS OF INFLATABLE DAMS UNDER OVERFLOW CONDITIONS

by

Pai-Hung Wu

Dissertation submitted to the Faculty of the

Virginia Polytechnic Institute and State University

in partial fulfillment of the requirements for the degree of Doctor of Philosophy

in

Civil Engineering

APPROVED:

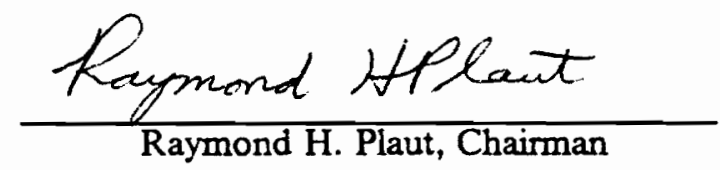

$\frac{\text { Richard M. Barker }}{\text { Richard M. Barker }}$

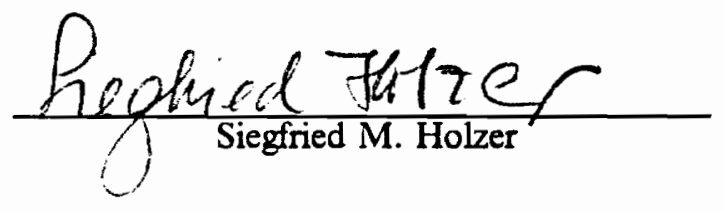

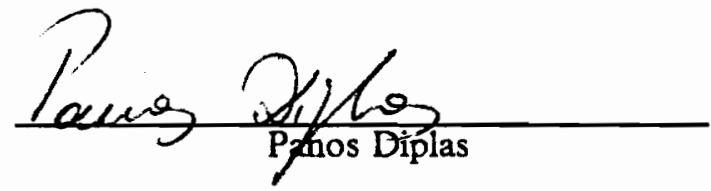

5. LiARS

May, 1995

Blacksburg, Virginia 
c. 2

LD

5655

$\checkmark 65$

1995

W8

$c^{2}$ - 


\title{
ANALYSIS OF THE VIBRATIONS OF INFLATABLE DAMS \\ UNDER OVERFLOW CONDITIONS
}

\author{
by \\ Pai-Hung Wu \\ Raymond H. Plaut, Chairman
}

Civil Engineering

(ABSTRACT)

\begin{abstract}
A two-dimensional analysis is applied to the vibrations of inflatable dams under overflow conditions. The static analysis yields the equilibrium state for both the free surface profile and the shape of the dam. The dynamic analysis investigates the small vibrations of the inflatable dam about the equilibrium state.
\end{abstract}

The dam is inextensible, air-inflated, and has two anchored points. The base width, curved perimeter, and internal air pressure are given. The overflow is incompressible, inviscid, and irrotational, and the total head is specified.

In the static analysis, the self-weight of the dam is neglected, and the equations of equilibrium from membrane theory are solved by a multiple shooting method. The boundary element method is used to solve Laplace's equation defined on the overflow domain. An iterative scheme is adopted to obtain the shape of the dam, as well as the location of the free surface.

From the equilibrium state, the dynamic analysis is established by a finite difference form of the membrane's equations of motion and the velocity potential problem is formulated by the boundary element method. After the eigenvalue problem is solved, the eigenvalues and eigenvectors obtained are employed to describe the vibrations of the dam. The effects of the dam's density and damping coefficient are illustrated. 


\section{Acknowledgements}

I would like to thank Dr. Plaut for his advice, patience, and assistance in this research and dissertation. Thanks go to Dr. Holzer, Dr. Rojiani, and Dr. Reddy for their instruction on the numerical methods. Much help was given by Dr. Diplas and Dr. Liapis on the problem of dams and hydrodynamics. I thank Dr. Barker for his willingness to join the committee as a replacement, to read the dissertation, and to make constructive comments. I also want to acknowledge financial support from the National Science Foundation under Grant No. MSM-9008518. 


\section{Table of Contents}

$\begin{array}{ll}\text { Chapter } 1 \text { INTRODUCTION } & 1\end{array}$

$\begin{array}{ll}\text { Chapter } 2 \text { LITERATURE REVIEW } & 6\end{array}$

2.1 REVIEW OF SOME RAPIDLY-VARIED FLOW PROBLEMS 6

2.2 REVIEW OF STUDIES ON INFLATABLE DAMS 10

Chapter 3 STATIC ANALYSIS

3.1 INTRODUCTION TO STATIC ANALYSIS 14

3.2 THE POTENTIAL FLOW PROBLEM 15

3.3 GOVERNING EQUATIONS FOR THE MEMBRANE 18

$\begin{array}{ll}3.4 \text { POTENTIAL FLOW ANALYSIS } & 20\end{array}$

3.5 THE ALTERNATING ITERATION AND AN EXAMPLE 23

$\begin{array}{ll}\text { Chapter } 4 \text { DYNAMIC ANALYSIS } & 38\end{array}$

4.1 INTRODUCTION TO DYNAMIC ANALYSIS 38

4.2 EQUATIONS OF MOTION FOR THE MEMBRANE 39

4.3 FLOW DOMAIN ANALYSIS $\quad 46$

4.4 EQUATION ASSEMBLING 
4.5.1 Example: $\bar{\rho}=1.0 ; \bar{\beta}=0.1$

4.5.2 Example: $\bar{\rho}=12 ; \bar{\beta}=1$

4.5.3 Example: $\bar{\rho}=50 ; \bar{\beta}=1 \quad 58$

4.5.4 Variation of $\lambda$ with $\bar{\beta} \quad 59$

$\begin{array}{ll}\text { 4.5.5 Variation of } \lambda \text { with } \bar{\rho} & 60\end{array}$

$\begin{array}{ll}\text { Chapter } 5 \text { CONCLUSIONS AND SUGGESTIONS } & 102\end{array}$

$\begin{array}{ll}5.1 \text { CONCLUSIONS } & 102\end{array}$

$\begin{array}{ll}5.2 \text { SUGGESTIONS } & 103\end{array}$

$\begin{array}{ll}\text { REFERENCES } & 104\end{array}$

APPENDIX A: THE FINITE DIFFERENCE SCHEME 107

A.1 FINITE DIFFERENCE FOR EQUATION (4.23) 107

A.2 FINITE DIFFERENCE FOR EQUATION (4.55) 110

A.3 FINITE DIFFERENCE FOR EQUATION (4.66) 112

$\begin{array}{ll}\text { Vita } & 114\end{array}$ 


\section{List of Illustrations}

Figure 1.1 An Inflatable Dam under Overflow 4

Figure 1.2 A Two-dimensional Cross-section 5

Figure 3.1 A Membrane Element in Equilibrium $\quad 30$

Figure 3.2 The Flow Domain 31

Figure 3.3 BEM Node Setup $\quad 32$

Figure 3.4 Result at Iteration Cycle 100

Figure 3.5 Nodes on Free Surface with Bernoulli Error over 3\% 34

Figure 3.6 Pressure Distribution on Dam Surface $\quad 35$

Figure 3.7 Error Function on the Free Surface with Iteration Cycle 36

Figure 3.8 Velocity Potential at Downstream Outflow Surface with Iteration Cycle 37

$\begin{array}{ll}\text { Figure 4.1 A Membrane Element in Vibration } & 61\end{array}$

Figure 4.2 Membrane Node Setup $\quad 62$

Figure 4.3 The Flow Domain in Dynamic Analysis 63

Figure 4.4 Example 4.5.1, Mode 1, Displacement with Time 64

Figure 4.5 Example 4.5.1, Contribution of Real Parts of Eigenvectors 65 
Figure 4.6 Example 4.5.1, Contribution of Imaginary Parts of Eigenvectors

Figure 4.7 Example 4.5.2, Mode 1, Displacement with Time (First Cycle) 67

Figure 4.8 Example 4.5.2, Mode 1, Displacement with Time (Second Cycle) 68

Figure 4.9 Example 4.5.2, Mode 1, Displacement with Time (Third Cycle) 69

Figure 4.10 Example 4.5.2, Mode 1, Displacement with Time (Fourth Cycle) 70

Figure 4.11 Example 4.5.2, Mode 1, Displacement with Time (Fifth Cycle) 71

Figure 4.12 Example 4.5.2, Mode 2, Displacement with Time 72

Figure 4.13 Example 4.5.2, Mode 3, Displacement with Time 73

Figure 4.14 Example 4.5.2, Mode 4, Displacement with Time 74

Figure 4.15 Example 4.5.2, Mode 1, Radial Displacement at Node $27 \quad 75$

Figure 4.16 Example 4.5.2, Mode 1, Radial Displacement at Node $22 \quad 76$

Figure 4.17 Example 4.5.2, Mode 1, Radial Displacement at Node $17 \quad 77$

Figure 4.18 Example 4.5.3, Mode 1, Displacement with Time 78

Figure 4.19 Example 4.5.3, Mode 2, Displacement with Time 79

Figure 4.20 Example 4.5.3, Mode 3, Displacement with Time 80

Figure 4.21 Example 4.5.3, Mode 4, Displacement with Time $\quad 81$

Figure 4.22 Example 4.5.3, Mode 5, Displacement with Time 82

Figure 4.23 Example 4.5.3, Mode 3, Radial Displacement at Node $27 \quad 83$

Figure 4.24 Example 4.5.3, Mode 3, Radial Displacement at Node $22 \quad 84$

Figure 4.25 Example 4.5.3, Mode 3, Radial Displacement at Node $17 \quad 85$

Figure 4.26 Example 4.5.3, Mode 1, Radial Displacement at Node 22 86

Figure 4.27 Example 4.5.3, Mode 2, Radial Displacement at Node $22 \quad 87$

Figure 4.28 Example 4.5.3, Mode 4, Radial Displacement at Node $22 \quad 88$

Figure 4.29 Example 4.5.3, Mode 5, Radial Displacement at Node 22

Figure 4.30 Imaginary Parts of Eigenvalues with $\bar{\beta}$ as $\bar{\rho}=1 \quad 90$

Figure 4.31 Real Parts of Eigenvalues with $\bar{\beta}$ as $\bar{\rho}=1 \quad 91$

Figure 4.32 Imaginary Parts of Eigenvalues with $\bar{\beta}$ as $\bar{\rho}=5 \quad 92$

Figure 4.33 Real Parts of Eigenvalues with $\bar{\beta}$ as $\bar{\rho}=5$ 
Figure 4.34 Imaginary Parts of Eigenvalues with $\bar{\beta}$ as $\bar{\rho}=12$

Figure 4.35 Real Parts of Eigenvalues with $\bar{\beta}$ as $\bar{\rho}=12$

Figure 4.36 Imaginary Parts of Eigenvalues with $\bar{\rho}$ as $\bar{\beta}=0.1$

Figure 4.37 Real Parts of Eigenvalues with $\bar{\rho}$ as $\bar{\beta}=0.1$

Figure 4.38 Imaginary Parts of Eigenvalues with $\bar{\rho}$ as $\bar{\beta}=1$

Figure 4.39 Real Parts of Eigenvalues with $\bar{\rho}$ as $\bar{\beta}=1$

Figure 4.40 Imaginary Parts of Eigenvalues with $\bar{\rho}$ as $\bar{\beta}=10$

Figure 4.41 Real Parts of Eigenvalues with $\bar{\rho}$ as $\bar{\beta}=10$ 


\section{List of Tables}

Table 3.1 Result at Iteration Cycle 100 (Part 1 of 2)

Table 3.1 Result at Iteration Cycle 100 (Part 2 of 2)
28

29 


\section{Chapter 1}

\section{INTRODUCTION}

Inflatable dams were invented by N. M. Imbertson in the 1950s with the trade name of "Fabridam" and have been used worldwide extensively ever since. About two thousand inflatable dams have been constructed in the United States, Japan, Thailand, Taiwan, and many other countries.

This type of dam is basically a flexible tube, made of rubberized fabric, fixed along its longitudinal base across a river, and inflated by air, water, or a combination of the two. It can be inflated to the required height or deflated on the foundation when not in use.

At the present time, the height of an inflatable dam can be up to 17.8 feet and the length can be up to 393.7 feet (Bridgestone, 1991). The life of serviceability is expected to be over 30 years while the cost of design, installation, and maintenance is considered to be lower than that of ordinary earth or concrete dams.

The applications of inflatable dams include tidal barriers, wave attenuators, flashboard replacements and reservoir providers. They are used to restrain mud, tide, flood, and salinity, to prevent beach erosion, to handle combined sewage flows, to divert underground flows, to regulate tunnel surge 
and tailwater, to serve as emergency check valves, to raise heights of existing spillway, to increase storage capacity, to raise intake head, to maintain lake level, and to impound water for irrigation, plant cooling, pumping station, and recreational purposes. Installing these dams on the top of an existing dam or spillway or replacing a damaged dam by an inflatable dam is fairly common.

Inflatable dams may be operated under conditions with or without overflow. The overflow condition is considered more likely to cause the vibrations of inflatable dams and therefore the stability problem. Bridgestone Engineered Products Co. limits the maximum overflow water height of their standard rubber dam to be two-fifths of the dam height (Bridgestone, 1991). The collapse of the inflatable dams in Pakistan in 1967 (Binnie et al., 1974) and Australia in 1969 (University of Sidney, Civil Engineering Laboratories, 1969) under overflow conditions indicates that the study of the vibrations is indispensable.

In this study, the inflatable dam is inflated with air and fixed at two anchored lines along its longitudinal direction (Figure 1.1). The vibrations of inflatable dams under overflow conditions are treated as a two-dimensional problem (see Figure 1.2 ) with the base width $\left(l_{0}\right)$, the curved perimeter $\left(s_{0}\right)$, the inflated air pressure $\left(q_{0}\right)$, and the total head for the flow domain $\left(e_{0}\right)$ given. The dam is described as an inextensible membrane and the vibrations are assumed to be small. The overflow domain is regarded as a hydrodynamic condition with subcritical flow upstream and supercritical flow downstream. The flow is bounded by a level bed, the inflatable dam, and a free surface with atmospheric pressure. The velocity potential theory prevails in the overflow domain.

The equilibrium state is obtained with the help of the boundary element method and a multiple shooting method. The shape of the dam and the profile of the free surface are obtained as the result of the static analysis. The vibrations about the equilibrium state in the dynamic analysis are investigated using the finite difference forms of the equations of motion for the dam and the boundary element method for the flow domain. Examples with various damping coefficients and water/dam density ratios are demonstrated. The eigenvalues and eigenvectors serve to depict the vibrations of the inflatable dams. 
Conclusions and suggestions are presented in the last chapter. It is the goal of this study that the vibrations of inflatable dams under overflow conditions can be better understood, further research can be pursued, and assistance can be provided for the design codes, construction process, and maintenance work of the inflatable dams. 


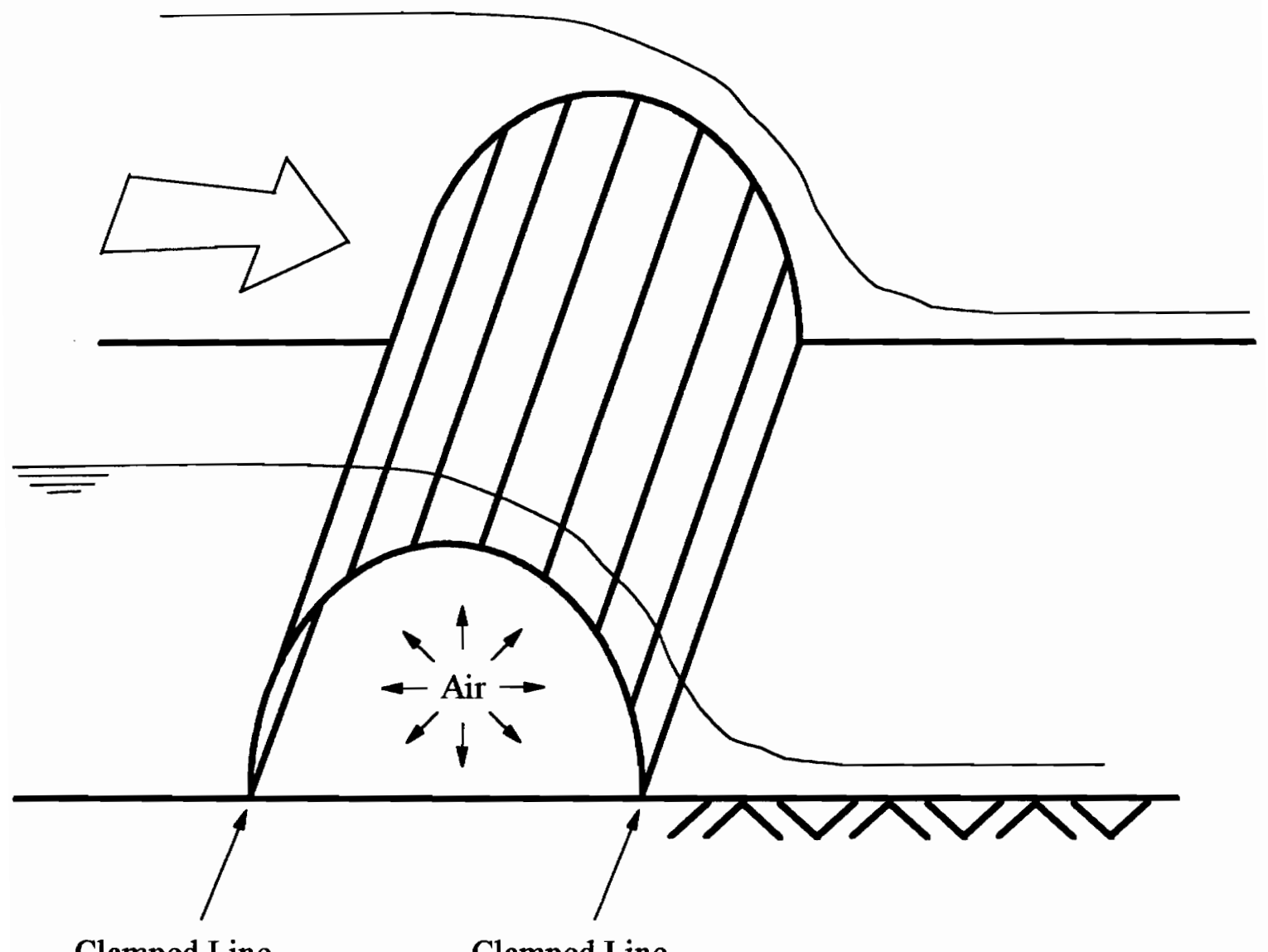

Clamped Line

Clamped Line

Figure 1.1 An Inflatable Dam under Overflow 


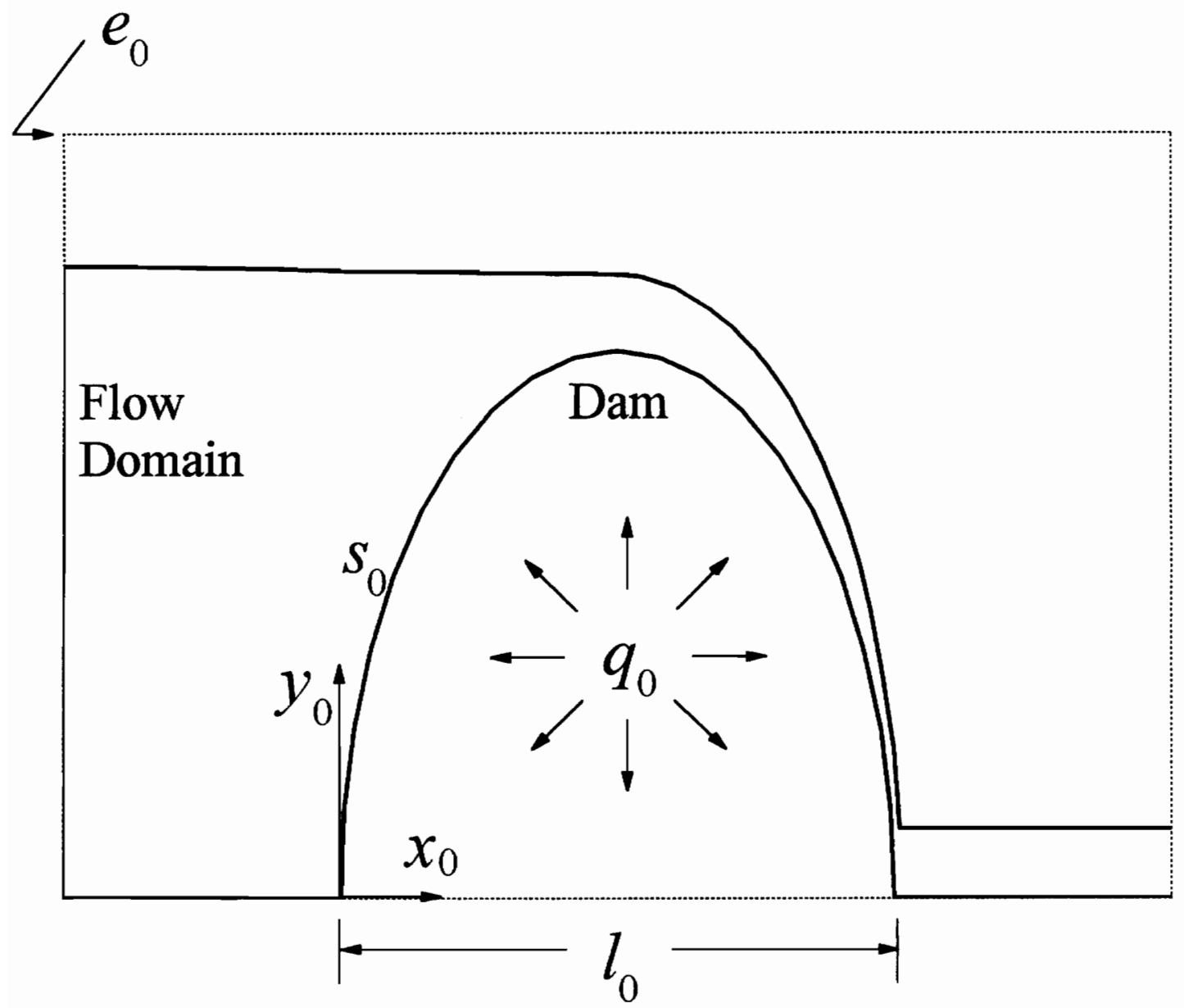

Figure 1.2 A Two-dimensional Cross-section 


\section{Chapter 2}

\section{LITERATURE REVIEW}

The overflow condition for inflatable dams is considered similar to the cases where flow passes over cylinders, broad-crested weirs, and spillways. Section 2.1 is a review devoted to the steady solutions of such rapidly-varied flow problems. On the other hand, the developments in the analyses of inflatable dams, either for static analysis or dynamic analysis, are presented in Section 2.2 .

\subsection{REVIEW OF SOME RAPIDLY-VARIED FLOW PROBLEMS}

The rapidly-varied flow problems, such as the flow over weirs, spillways, and other kinds of obstacles, are characterized by the influence of gravity and the unknown location of the free surface. The governing equation for such flows in a steady sense is an elliptic partial differential equation. With the total energy head given, the flow rate is solved as part of the solution, or vice-versa.

It was pointed out by von Karman (1940) that the analysis of gravity-driven free-surface flows carries inherent difficulties not only from the nonlinear character of the boundary condition, but also from the fact that the boundary is not known a priori. Southwell and Vaisey (1946) traced the 
location of the initially unknown free streamline, where a double boundary condition applies, by finite differences (relaxation methods) with hand calculation. Two iterative methods were introduced by making the value of the stream function constant or by specifying the gradient of the stream function on the assumed free surface, respectively. It was claimed that an essential instability makes any tentative solution liable to diverge and that abnormally fine nets are necessitated by the rapid curvature of free streamlines. The finite differences employed by Cassidy (1965) are applied to the slopes at the nodes instead of the stream functions at the nodes. A rectangular mesh can be established by mapping the physical boundaries into the complex-potential plane. It was observed that the computed free surface ordinates became progressively smaller when the flow rate or total head was too large. An opposite trend occurred when the flow rate or total head was too small.

In terms of the velocity potential, Luke (1967) presented a variational principle for a fluid with a free surface. The Lagrangian in his functional was equal to the pressure term, which leads to a full set of boundary conditions for the classical water wave problem. This Lagrangian is also compared with the more usual expression which results from the difference of kinetic energy and potential energy. Chan et al. (1973) employed a variational principle to solve the Laplace's equation for a sharp slot flow. On the free surface, the velocity potentials are specified according to the Bernoulli equation and the normal velocities are calculated. From the tangential and normal velocities, the slopes at the free surface nodes are determined. Then, a series of cubic polynomials is adopted to fit through each set of three consecutive nodal points, which yields the adjustment of the free surface location. To minimize computational cost, a low initial free surface is recommended. Ikegawa and Washizu (1973) applied a special case of Luke's variational principle to a spillway problem, and used the finite element method. The flow in a spillway problem is subcritical upstream and supercritical downstream. There exists a transition region (the so-called control section) near the crest where mathematical difficulty may occur. They found that Southwell and Vaisey's two iterative methods can be used to obtain the subcritical and supercritical regions, but not the transitional region. Their experience in numerical computation shows that if the flow rate is larger than the 
exact solution, the free surface converges to a profile with broken segments near the crest. If the flow rate is smaller than the exact solution, the free surface displays a divergent behavior near the crest. The flow rate which causes the smoothest convergent free surface profile is taken as the solution.

Varoglu and Finn (1978) developed a variational principle that preserves the nonlinear free surface boundary conditions by adding two free surface integral terms to the first variation of the kinetic energy. The iteration scheme is an inverse finite element procedure in which the location of a set of streamlines with assumed stream function values is to be calculated. Thus, the addition or deletion of nodes and elements, which may happen in Ikegawa and Washizu's formulation, can be avoided and the flow rate is able to be obtained directly from the iterations. Betts (1979) explained that Luke's variational principle is less well suited to problems of steady flow under the influence of gravity, since the values of velocity potential on the free surface boundary are not known beforehand. Therefore the formulation in terms of stream function is more convenient to solve a twodimensional steady flow problem. It was also found that Ikegawa and Washizu incorrectly neglected an integral along the free surface in Green's identity. Betts used a functional in the form of kinetic energy minus potential energy for the variational principle. A combination of this variational principle and an iteration method similar to Ikegawa and Washizu's (performed by finite elements) was claimed to work well regardless whether the Froude number of the flow is less or greater than unity, or only a crude initial guess of the free surface is available.

Bettess and Bettess (1983) used the same functional as Betts in the variational principle and applied isoparametric elements for the finite element mesh. The mesh allows the free surface and the bed to be curved rather than piecewise straight. Like Ikegawa and Washizu's work, the flow rate which yields the smoothest free surface profile was selected as the solution, which also corresponded to a minimum of the square of the error in the Bernoulli equation integrated along the free surface in their study. Underestimates of the depth in supercritical flow were found always to converge, as do overestimates in subcritical flow. Overestimates of the depth in supercritical flow, however, might tend towards the subcritical solution. Castro-Delgado and Celik (1986) considered that Betts 
ignored the term for the variation of the stream function at a space-fixed point. They added one free surface integral term to the first variation of the functional of kinetic energy and potential energy, which results in the same variational principle as in Varoglu and Finn (1978). The principle was applied to the flows past overflow gates in which a free jet trajectory exists, by the inverse finite element method devised by Varoglu and Finn. It is shown that the influence of viscosity can be neglected in this class of rapidly accelerating flows.

Cheng et al. (1981) made use of the boundary integral equation method and perturbation-based iteration schemes to solve a sluice gate and a spillway problem. For the spillway, there is a zone of uncertainty where a change in the water surface level has a negligible effect on the Bernoulli values. A perturbation matrix was established by displacing, in turn, each free surface node a small distance from its assumed value and computing the change in the Bernoulli values at all free surface nodes due to this displacement. Then the adjustment of the free surface was determined from the perturbation matrix and the errors in Bernoulli values of the free surface nodes by a NewtonRaphson method. Some pseudo-nodes were inserted between the free surface nodes with the elevation and velocity interpolated, for the perturbation matrix, so that the error in the Bernoulli constant was minimized at a larger number of nodes instead of only at the real nodes. The free surface was adjusted only at the real nodes with some limits or damping in order to make the adjustment always reasonable. Jovanovic (1987) followed Cheng et al. and used linear elements and pseudo-nodes along the free surface in the boundary element formulation. A step factor or damping factor applied to the elevation adjustment of the free surface appeared to be problem- dependent and was determined empirically. An automatic adjustment of this step factor at each iteration would improve the rate of convergence. In one of the cases studied, there is an averaged 10 percent error on flow velocity after 150 iterations with a step factor of 0.2 . For the same case, no convergence could be obtained after 200 iterations. It was pointed out that the gradient method might be an alternative approach when it was employed on an error function which is the sum of the squares of the errors in the Bernoulli constant at all the free surface nodes. 
For a semi-circular obstacle, Forbes (1988) worked on the domains of stream function and velocity potential instead of $\mathrm{x}$ and $\mathrm{y}$-coordinates for the free surface problem. Complex variables, conformal mapping, Newton's method, and the integral equation derived by Forbes and Schwartz (1982) were employed. The variations of the downstream speed, the upstream Froude number and the profile of the free surface with the radius of the semi-circle were illustrated. A difficulty in the convergence of solution was pointed out when the radius (or height) of the semi-circular obstacle was more than half the far upstream inflow depth. It was also the case that the downstream portion of the flow became shallow and fast, which could cause the formation of a hydraulic jump.

\subsection{REVIEW OF STUDIES ON INFLATABLE DAMS}

The inflatable dams are usually considered as membrane structures with the resistance in the form of tension. The loads applied on the inflatable dams include the internal inflated pressure (air, water, or their combination) and the external water or atmospheric pressure. The external water pressure can be hydrostatic pressure (without overflow) or hydrodynamic pressure (in overflow conditions).

Anwar (1967) performed the calculations for water-inflated dams under hydrostatic conditions, and air-inflated dams under both hydrostatic and steady overflow conditions. The results were compared with an experimental model investigation. Under the assumptions that the self-weight of the dam was negligible and the inflated pressure was proportional to the storage head, the hydrostatic shape of an inflatable dam (inflated by air or water) was derived partly in terms of elliptic integrals and partly in terms of an equation of a circle from the equilibrium of forces. No restrictions were set for the perimeter and the base length of the dam. For the hydrodynamic case, the flow on the downstream face of the air-inflated dam was assumed to be fully aerated. Therefore, the pressure applied on the downstream face due to the overflow was atmospheric and the downstream dam shape was part of a circle. By a set of power series approximations for the vertical coordinate, the pressure, and the horizontal force distribution, an ordinary differential equation was established and numerically solved for the upstream dam shape. A parabola was found to be a good approximation 
in the power series formulation. A model test was set up with the height of the dam equal to 12 in. in the static case and 9 in. in the overflow case. A trip rod or wire placed near the crest of the dam prevented the flow from clinging to the downstream face and reduced the skin vibration considerably, provided the nappe was fully aerated. There was no sign of flow separation when the dam was operating without a trip wire, in which water followed the profile of the dam over the major part of the downstream face. It was observed that an inflatable dam was not suitable for a high overflow condition.

Harrison (1970) studied air-inflated dams and water-inflated dams subjected to hydrostatic pressure from both the upstream and downstream heads. The two-dimensional membrane section was considered as composed of a finite number of small elements with concentrated loads acting on the ends of elements only. The base width and curved perimeter were specified. A Newton's method was employed to improve the shape of the dam and to match the specified positions of the anchored points. For a rising upstream head, an air-inflated dam was found to reduce the membrane tension more than a water-inflated dam. Air-inflated dams might entail more risk of an explosive failure if the membrane was damaged, but may be more economical. Binnie (1973) dealt with water-inflated dams impounding water at the crest level. Besides the problem of the unrestricted curved perimeter and base width, it was mentioned that the anchorages of the upstream and downstream faces should not be at different levels as in Anwar (1967). Assuming that the membrane was weightless and inextensible, a solution for the shape of the dam was obtained, with the upstream face being part of a circle and the downstream face expressed by an equation in terms of elliptic integrals, and this shape corresponded to the anchored points at the same level. The drawback is that the inverse formulation was not performed so that the curved perimeter and base width would be part of the solution instead of input quantities, while the tension in the membrane and the slope at the downstream anchored point were given.

Parbery (1976) derived the differential equations of equilibrium using membrane theory and solved those equations with a fourth-order Runge- Kutta method and the Newton-Raphson method for the inflatable darns under hydrostatic conditions. The self-weight and the modulus of elasticity of 
the membrane were considered, as well as the restrictions on the base width and the curved perimeter. In his second paper (Parbery, 1978), the weight and the elasticity of the membrane were found to have minor influences on the shape of the dams for the hydrostatic conditions. For a given base width, the major influences resulted from the inflated pressure, the inflation method, the impounded head, and the curved perimeter. Watson (1985) dealt with the theoretical calculation of the shapes of the water-inflated or air-inflated dams impounding water at the crest level (without overflow) in a way similar to Anwar (1967) and Binnie (1973). The weight and the modulus of elasticity were ignored. Design charts for various loading parameters and fabric length/anchorage spacing ratios were presented. Parachute dams in which the upper end of the membrane was fixed to a floating boom and restrained by guys were also discussed.

Fagan (1987) investigated the effect of the self-weight of the membrane on the vibrations of the air-inflated dams. For the equilibrium shape of the dams, a Runge-Kutta-Verner fifth and sixth order method and a bisection iteration algorithm were employed to solve the simultaneous ordinary differential equations derived from membrane theory in Parbery (1976). The analysis of vibrations was performed by applying an eigenvalue solution approach to the finite difference form of the equations of motion for the membrane under small vibrations. The weight of the membrane was found to have an insignificant impact on the static shape of the dams as long as the extensibility of the membrane was neglected. The tension in the membrane increased when the self-weight decreased or the base to perimeter length ratio increased. For the dynamic case, the weight of the dams tended to lower the tension and vibration frequencies for the membrane, but the effect was much less than other factors, such as the base width. The vibration modes were not affected by the weight of the dam significantly, as well (Plaut and Fagan, 1988).

Leeuwrik (1987) analyzed the vibrations of air-inflated dams by the Galerkin approximation with one term or two terms of sine functions, and a Runge-Kutta-Verner method. The dams were assumed to be weightless, inextensible, and sufficiently long so that a two-dimensional analysis was acceptable. In some cases, the inflatable dams were found not to oscillate about the equilibrium state, but about a position with some displacement from the equilibrium shape. The frequencies 
of vibrations were reduced by such a displacement, but not much (which was a result of the nonlinear terms in the equation of motion). Moreover, solutions were not guaranteed by the method, thus either more terms of approximation functions or an alternate solution routine was needed. A comparison between this numerical solution and the result of the asymptotic analysis (the method of multiple scales) was given in Plaut and Leeuwrik (1988). It was confirmed that the vibration frequencies tended to decrease as the amplitude of motion increased.

Hsieh (1988) considered the free vibrations of both air-inflated dams and water-inflated dams, and the forced vibrations of water-inflated dams impounding upstream water at the crest level, with the weight of the dams neglected. A finite difference method was used for the membrane equation of motion and the water domain was analyzed by a boundary element method. For the water-inflated dam without water outside, the vibration frequencies increased as the internal water head increased, the perimeter/base width ratio decreased, or the water/dam density ratio decreased (see also Hsieh et al., 1989). When there existed impounded water outside, the water/dam density ratio displayed similar effects on the vibration frequencies, but it was not always true that the external water head lowered the vibration frequencies. In Hsieh and Plaut (1990), it was further clarified that the frequencies increased when the internal and the external water heads increased simultaneously. If the external head increased with the internal head fixed, the frequencies decreased at the beginning, then some of them started to increase. 


\section{Chapter 3}

\section{STATIC ANALYSIS}

\subsection{INTRODUCTION TO STATIC ANALYSIS}

The static equilibrium shape of an inflatable dam under a steady overflow condition is to be calculated in this chapter.

The dam is assumed to be inextensible, with negligible weight. The governing equations for the shape of the dam are based on membrane theory and solved by a multiple shooting method. For a given base width (the linear distance between the fixed points in a two-dimensional point of view) and a given curved perimeter of an inflatable dam with given internal air pressure, the shape of the dam is determined as soon as the external pressure is known. The information on the external pressure comes from the result of the overflow analysis.

The overflow is treated as an incompressible, inviscid, and irrotational flow problem, in which Laplace's equation prevails. With the total head of flow given, the Laplace's equation and the corresponding boundary conditions are solved by a boundary element method if the location of the free surface is assumed and the boundary of the dam-flow interface (the shape of the dam) is known. 
The free surface can be found when the double boundary conditions are satisfied at the same time. Thus, the external pressure for the dam can be computed by the Bernoulli equation applied to the dam-flow interface.

An alternating iteration approach is used to perform the two iterations on the dam shape which supplies part of the boundaries for the flow analysis and the free surface to generate the external pressure for the dam analysis. The solution is considered to be reached when both the changes of the dam shape and the free surface in successive iterations are within preset tolerances.

\subsection{THE POTENTIAL FLOW PROBLEM}

For analyzing the hydrodynamic overflow problem, the overflow was first considered to be separated after passing the crest of the dam. Potential flow theory was applied in the overflow domain before the separation point and the static backwater prevailed after the separation point. This scheme was not successful due to the difficulty of locating the separation point and providing sufficient information in the backwater region for the vibration analysis. Therefore, the whole overflow domain is assumed to be described by Laplace's equation without any separation.

The overflow problem is a gravity-driven flow problem with double boundary conditions applying on the free surface. Moreover, sharp corners may exist at the upstream and downstream anchored points of the inflatable dams. Various solution schemes have been attempted, which involved the following:

1. Either the stream function or the velocity potential as the dependent variable in Laplace's equation;

2. The flow rate, the upstream inflow depth, the Bernoulli constant (the total head), or the velocity potential difference between the upstream inflow surface and downstream outflow surface as the given datum for the overflow domain; 
3. Dirichlet boundary conditions or Neumann boundary conditions for the free surface, upstream inflow surface, and downstream outflow surface (also mixed boundary conditions for the free surface);

4. The use of polynomials (third to tenth order), or sine and cosine functions (two to eight terms), for the profile of the free surface;

5. Direct iteration from the Bernoulli equation, which is similar to the two iterative methods introduced by Southwell and Vaisey (1946);

6. The cubic-polynomial-smoothing algorithm for the free surface adjustment of the sharp slot flow as used in Chan et al. (1973);

7. The iteration for the free surface with the help of the calculation of the perturbation matrix in Cheng et al. (1981).

There was no noticeable difference observed as convergence was concerned when using either the stream function or the velocity potential for Laplace's equation. The velocity potential is selected for the sake of consistency in the formulations of the static analysis and the dynamic analysis. The choice for the given datum of the overflow domain depended on the iteration scheme. Most studies use the flow rate or the Bernoulli constant (the total head), though Cassidy (1965) implied that not every total head will yield a convergent solution for a given shape of the obstacle. As for the types of boundary conditions, it was observed that a discontinuity of the velocity potential and its derivatives existed if mixed boundary conditions are used on the free surface (Dirichlet for upstream and Neumann for downstream); otherwise, the choice depends on the iteration method, too. The experience of using polynomials or other mathematical functions to describe the profile of the free surface showed no benefits because they generated an unnecessary wavy shape far upstream or far downstream (especially when using higher order terms) or were not capable of following the high curvature near the downstream anchored point.

The direct iteration from the Bernoulli equation for computing the free surface location is easy to employ, but may converge to the subcritical solution for both upstream and downstream regions (or, supercritical for both regions) while the solution needed is subcritical upstream and supercritical 
downstream. Besides, kinky shapes may occur and convergence is not guaranteed. The algorithm in Chan et al. (1973) is appropriate for subcritical flow problems, but abnormal slopes (positive slopes or rising elevations) were experienced when calculating the upstream profile of the free surface with this overflow condition. The perturbation matrix approach in Cheng et al. (1981) was tested with the original node configuration, as well as with various polynomials and other functions. It was found to be time-consuming to calculate the elements in the perturbation matrix when lifting the elevation of the free surface by a small amount, node by node. It was still unclear how to decide on the moment when the perturbation matrix did not change much and need not be recalculated, and how to modify the elevation changes of the free surface with some 'limits or damping' to make them 'always reasonable'. The divergence encountered by Jovanovic (1987) which followed Cheng et al. (1981) was experienced in this study, too. In some cases, an acceptable solution exists at a certain iteration cycle, but it becomes divergent in a later iteration cycle.

There were some studies performing finite element analysis together with a variational principle, e.g., Varoglu and Finn (1978), and Ikegawa and Washizu (1973), and claiming success in the free surface flow problem. Nevertheless, a variational principle applied to the boundary element method in this study would be difficult, although the boundary element method has the advantage of smaller number of nodes and less computing effort, compared with the finite element method. Gradient methods might be considered as promising (Jovanovic, 1987), but they would lead to a similar situation as for the perturbation matrix.

For this overflow problem, the conclusion was to use the boundary element method, with the velocity potential as dependent variable, the Bernoulli constant (total head) given, a Neumann boundary condition on the free surface, and a modified direct iteration method (described in Section 3.4). This procedure attempts to obtain calculation efficiency, as well as solution accuracy. 


\subsection{GOVERNING EQUATIONS FOR THE MEMBRANE}

In the two-dimensional analysis, the shape of the dam is an arc fixed at the two anchored points and has unit depth in the direction into the paper. This arc is expressed by a set of neighboring membrane elements with unit depth. Figure 3.1 shows a membrane element under consideration. The self-weight of the inflatable dam and the corresponding membrane elements is ignored. With small slope change between the two ends of the element assumed and the higher order terms neglected, the equations for the equilibrium of the forces are

$$
\begin{aligned}
& \frac{d \theta_{0}}{d s}=\frac{p_{0}-q_{0}}{t_{0}} \\
& \frac{d t_{0}}{d s}=0
\end{aligned}
$$

where $s$ is the curved coordinate; $\theta_{0}$ is the slope of the membrane; $p_{0}$ is the external pressure times the unit depth; $q_{0}$ is the internal inflated pressure times the unit depth; and $t_{0}$ is the uniform tension per unit depth for the membrane. The subscript 0 is used for the variables in the static analysis. From geometry, we have

$$
\begin{aligned}
& \frac{d x_{0}}{d s}=\cos \theta_{0} \\
& \frac{d y_{0}}{d s}=\sin \theta_{0}
\end{aligned}
$$

in which $d x_{0}$ and $d y_{0}$ are the projections of the membrane element onto the $x$ and $y$ axes, respectively.

With $s_{0}$ being the given curved perimeter, $l_{0}$ being the given base width of the dam (see Figure 1.2), and $y$ being the specific weight of the fluid in the overflow, the following nondimensional variables are introduced: 
$\bar{s}=\frac{s}{b_{0}} ; \quad \bar{s}_{0}=\frac{s_{0}}{l_{0}} ; \quad \bar{x}_{0}=\frac{x_{0}}{l_{0}} ; \quad \bar{y}_{0}=\frac{y_{0}}{l_{0}}$

$\bar{\theta}_{0}=\theta_{0} ; \quad \bar{t}_{0}=\frac{t_{0}}{\gamma l_{0}^{2}} ; \quad \bar{p}_{0}=\frac{p_{0}}{\gamma l_{0}} ; \quad \bar{q}_{0}=\frac{q_{0}}{\gamma l_{0}}$

The nondimensionalized forms of Equations (3.1)-(3.4) are

$\frac{d \bar{\theta}_{0}}{d \bar{s}}=\frac{\bar{p}_{0}-\bar{q}_{0}}{\bar{t}_{0}}$

$\frac{d \bar{t}_{0}}{d \bar{s}}=0$

$\frac{d \bar{x}_{0}}{d \bar{s}}=\cos \bar{\theta}_{0}$

$\frac{d \bar{y}_{0}}{d \bar{s}}=\sin \bar{\theta}_{0}$

There are two fixed ends for the membrane, and the boundary conditions for Equations (3.6)-(3.9) are

$\bar{x}_{0}(0)=0 ; \quad \bar{y}_{0}(0)=0 ; \quad \bar{x}_{0}\left(\bar{s}_{0}\right)=1 ; \quad \bar{y}_{0}\left(\bar{s}_{0}\right)=0$

The four simultaneous ordinary differential equations accompanied by the four boundary conditions (Equations (3.6)-(3.10)) can be solved if $\bar{p}_{0}$ is known (either from an initial guess or the result of the flow analysis). We use a multiple shooting method named DBVPMS in IMSL (IMSL, 1989) in which Runge-Kutta-Verner fifth-order and sixth-order methods, Jacobian evaluation, and Newton's method are employed. The initial guess for DBVPMS will be discussed further in Section 3.5 . 


\subsection{POTENTIAL FLOW ANALYSIS}

The problem of an overflow passing over an inflatable dam is similar to the one with overflow passing over a spillway crest, which is characterized such that the flow in the upstream portion is subcritical, while the flow in the downstream portion is supercritical (e.g., see Ikegawa and Washizu, 1973). For a given total head and a specified dam shape, the profile of the free surface (including the subcritical and supercritical transition phenomenon) and the flow rate are part of the solution.

In order to do the flow domain analysis, there are two fictitious boundaries which need to be specified: the upstream inflow and the downstream outflow boundaries. According to Hsieh (1988), the upstream boundary has to be several times the base width away from the dam to cause good convergent results in the dynamic analysis. In the case of the overflow here, the upstream inflow and downstream outflow boundaries are set at six times the base width away from the dam instead of five times as in Hsieh's work. The domain of the flow is displayed in Figure 3.2. Laplace's equation is adopted to describe the overflow, so that

$\nabla^{2} \phi_{0}=0 \quad$ in Flow Domain

where $\nabla^{2}=\frac{\partial^{2}}{\partial x^{2}}+\frac{\partial^{2}}{\partial y^{2}}$ and $\phi_{0}$ is the velocity potential for the flow. The boundary conditions are

$\phi_{0}=0 \quad$ on Inflow Boundary

$\phi_{0}=\phi_{0 d} \quad$ on Outflow Boundary

$\phi_{0, n}=0 \quad$ on Bed Bottoms and on Dam - Flow Interface

$\phi_{0, n}=0 \quad$ on Free Surface

$y_{0}+\frac{\rho\left(\phi_{0, s}\right)^{2}}{2 \gamma}=e_{0} \quad$ on Free Surface 
where $n$ is the unit outward normal to the surface enclosing the flow domain; $y_{0}$ is the $y$-coordinate of the free surface; $\phi_{0, s}$ is the flow velocity along a streamline; $\rho$ is the density of the fluid in the flow, which equals the specific weight divided by the acceleration of gravity, $\frac{\gamma}{g}$; and $e_{0}$ is the specified total head. Equations (3.12) and (3.13) indicate that the upstream inflow and the downstream outflow velocities are perpendicular to the inflow and outflow cross-section, respectively. The uniform velocity potential is chosen to be zero at the inflow boundary and $\phi_{0 d}$ at the outflow boundary, where $\phi_{0 d}$ is part of the solution. Equation (3.14) shows that the impenetrable fixed bed boundaries and dam-flow interface are part of streamlines. In Equation (3.15), the free surface is also part of a streamline so that $\phi_{0, n}=0$; moreover, it is subjected to constant atmospheric pressure (set to zero) and carries a simpler form of the Bernoulli equation (Equation (3.16)). Double boundary conditions are observed at the free surface.

The following nondimensional variables are introduced:

$\bar{\rho}=\frac{l_{0}}{\mu} \rho ; \quad \bar{n}=\frac{n}{l_{0}} ; \quad \bar{s}=\frac{s}{l_{0}} ; \quad \bar{y}_{0}=\frac{y_{0}}{l_{0}} ; \quad \bar{e}_{0}=\frac{e_{0}}{l_{0}} ; \quad \bar{\phi}_{0 d}=\sqrt{\frac{\mu}{\gamma}} \frac{1}{l_{0}^{2}} \phi_{0 d} ;$

$\bar{\phi}_{0}=\sqrt{\frac{\mu}{\gamma}} \frac{1}{l_{0}^{2}} \phi_{0} ; \quad \bar{\phi}_{0, \bar{s}}=\sqrt{\frac{\mu}{\gamma}} \frac{1}{l_{0}} \phi_{0, s} ; \quad \bar{\phi}_{0, \bar{n}}=\sqrt{\frac{\mu}{\gamma}} \frac{1}{l_{0}} \phi_{0, n}$

where $\mu$ is the mass per unit area of the membrane, which is a constant. Equations (3.11)-(3.16) become

$\nabla^{2} \bar{\phi}_{0}=0 \quad$ in Flow Domain

$\bar{\phi}_{0}=0 \quad$ on Inflow Boundary

$\bar{\phi}_{0}=\bar{\phi}_{0 d} \quad$ on Outflow Boundary

$\bar{\phi}_{0, \bar{n}}=0 \quad$ on Bed Bottoms and on Dam - Flow Interface

$\bar{\phi}_{0,}, \bar{n}=0 \quad$ on Free Surface 
$\bar{y}_{0}+\frac{\bar{\rho}\left(\bar{\phi}_{0,}\right)^{2}}{2}=\bar{e}_{0} \quad$ on Free Surface

For a given free surface and a velocity potential at the outflow boundary, $\bar{\phi}_{0 d}$ (either from the initial guess or the result of the previous iteration), Equations (3.18)-(3.22) form a complete set of the potential problem. The potential problem is solved by a boundary element method using linear elements (Liggett and Liu, 1983) so that the velocity potential at the free surface is obtained. The flow velocity along the free surface $\left(\bar{\phi}_{0,}\right)$ can be calculated from the velocity potential by a finite difference method, which usually does not satisfy the Bernoulli equation (Equation (3.23)) simply because it does not come from the correct free surface. For the free surface location, $\bar{y}_{0}$, and the result of the boundary element method applied to Equations (3.18)-(3.22), the flow velocity obtained is $\bar{u}_{1} ;$ for the same free surface location, the flow velocity calculated directly from Equation (3.23) is $\bar{u}_{0}\left(=\bar{\phi}_{0, s}\right)$. We have

$\bar{u}_{2}=\bar{u}_{1}+\frac{\bar{u}_{1}-\bar{u}_{0}}{\alpha} \quad$ for Subcritical Flow

$\bar{u}_{2}=\bar{u}_{0}+\frac{\bar{u}_{0}-\bar{u}_{1}}{\alpha} \quad$ for Supercritical Flow

where $\bar{u}_{2}$ is the flow velocity to be used in Equation (3.23) to compute the new $\bar{y}_{0}$ for the next iteration; and $\alpha$ is an adjustment parameter, in general between 10 and 30 (Li et al., 1989). The location where the flow is divided into the subcritical and supercritical regions is determined by the Froude number:

$F_{r}=\frac{\bar{u}_{1}}{\sqrt{\bar{d}}}$

where $\bar{d}$ (nondimensionalized by $l_{0}$ ) is the difference between the elevation of a free surface node and that of another node which carries the same value of velocity potential but is located at the bed bottom or at the dam/flow interface (e.g., Chow, 1959). Chow used somewhat different formulas 
for the energy coefficient (which was applied to the velocity term) to account for logarithmic or linear velocity distribution along the depth of water. It is found that using the energy coefficient does not improve the convergence in this study, so the simpler form (Equation 3.26) is adopted. Starting from the most upstream node on the free surface, the first node with the Froude number which is greater than unity is taken as the critical point. By experience, the elevation change of the calculated free surface has to be multiplied by a deduction factor to keep the change small, and a smooth curve-fitting may be used on those nodes near the critical point determined by Equation (3.26), especially at the first several iterations.

The external pressure for the dam at the dam/flow interface, from the Bernoulli equation, is

$\bar{p}_{0}=\bar{e}_{0}-\bar{y}_{0}-\frac{\bar{\rho}\left(\bar{\phi}_{0, s}\right)^{2}}{2}$

\subsection{THE ALTERNATING ITERATION AND AN EXAMPLE}

The alternating iteration method applied here is basically performing an iteration once for the dam shape immediately after one iteration for the free surface is executed. An initial guess for both the free surface and the dam shape has to be established before the iterations begin. The following example can serve to demonstrate how the alternating iteration works.

We have an inflatable dam with $\bar{s}_{0}$ equal to $\frac{\pi}{2}$, and inflated by an air pressure term, $\bar{q}_{0}$, of $2 \bar{e}_{0}$ (twice the total head). The base of the inflatable dam carries unit width and is anchored at $\bar{x}_{0}=0$ and $\bar{x}_{0}=1$ in the two-dimensional point of view (Figure 3.3). The fictitious upstream and downstream boundaries for the inflow and outflow of the fluid domain are set at $\bar{x}_{0}=-6$ and $\bar{x}_{0}=7$, respectively, which are both six times the base width away from the anchored points of the dam. The total head (Bernoulli constant) is given as $\bar{e}_{0}=0.6060$.

Before the first iteration takes place, we assume the initial guess for the dam shape to be half a circle, which is the shape for the dam under the condition of the internal inflated pressure acting alone. 
For the free surface profile, the initial guess looks like that in Figure 3.3, with the upstream inflow depth higher than that of the downstream outflow (subcritical and supercritical, respectively).

A hundred nodes are distributed along the boundary of the fluid domain for the boundary element analysis, while 21 of them also belong to the configuration of the membrane structure ( 20 elements) for the multiple shooting method (the subroutine DBVPMS in IMSL, 1989). The one hundred nodes are clockwise numbered as $2,3, \ldots, 101$ just because of the convenience for the programming of the boundary element method, where Nodes 12-32 are on the dam surface (Figure 3.3). Node numbers are indicated by being enclosed with small circles. In the bottom half of Figure 3.3, only those nodes which fall into the range between $\bar{x}_{0}=-0.5$ and $\bar{x}_{0}=1.5$ are shown. The distances between consecutive nodes are smaller where the curvatures are larger. Not many nodes are found near the two sharp corners (anchored points) because they are replaced with four special elements (see next paragraph).

The boundary element method used is a modified version of the programs "GM8" and "GM9" in Liggett and Liu (1983). The modification made on "GM8" is to include the codes for the Neumann Problem where the velocity potential is not uniquely determined, and the codes allow the formulation for the nodes with the velocity potentials unknown but the outward normal derivatives of the velocity potentials at both sides given and discontinuous. The modification done to "GM9" is to expand the use of the 16-point Gaussian quadrature in six panels for a single element (Liggett and Liu, 1983) for sharp comers of any angle. In this work, the boundary elements used for the fluid domain are linear elements with the exception of four special elements at both sides of the anchored points (sharp corners with angles to be determined).

The alternating iteration method uses a number of iteration cycles. Each iteration cycle consists of an iteration for the free surface elevation followed by an iteration for the dam shape. By the boundary element method and the free surface adjustment scheme displayed in Section 3.4, the iteration for the free surface in each cycle is operated with the dam shape fixed. The external pressure for the dam then can be calculated by Equation (3.27). On the other hand, with the free surface 
fixed and the external pressure information, we can perform the iteration for the dam shape as in Section 3.3 by the multiple shooting method, and obtain the new dam shape for the next iteration cycle.

When the alternating iterations are performed, it is noticed that the adjustments made either for the elevations of the free surface nodes ( $\mathrm{x}$-coordinates fixed) or for the displacements for $\mathrm{x}$ and $\mathrm{y}$ coordinates of the membrane nodes have to be scaled down at most iterative cycles. Otherwise, the multiple shooting method may fail (no solution for the state of the external pressure distribution) and the free surface may exceed the total head, penetrate the dam, fall below the bed level, or have large kinks. In this example, a scaling factor of $\frac{i t e r}{100}$ is used for all the coordinate changes, where iter is the sequence number of the iteration cycle. The scaling factor is kept less than or equal to one, and the value equals one after the 100th iteration cycle (no scale-down). Besides, an exponential form is employed whenever there is a small region of discontinuity around the crest at the free surface and smoothing is needed.

The initial guess and the result at the 100th iteration cycle are detailed in Table 3.1. An initial guess of the velocity potential at the downstream outflow surface, $\bar{\phi}_{0 d}$, is made by successively applying the Bernoulli equation over the free surface elements:

$\bar{\phi}_{0 d}=\sum_{i=52}^{91} \Delta \bar{s}_{i} \sqrt{2 \bar{e}_{0}-\bar{y}_{0 i}-\bar{y}_{0 ; i+1}}$

where $\bar{y}_{0 i}$ and $\bar{y}_{0 ; i+1}$ are the y-coordinates of Nodes $i$ and $i+1$, respectively; $\Delta \bar{s}_{i}$ is the distance between Nodes $i$ and $i+1 ; 52$ and 91 are the first and the last element numbers on the free surface. Equation (3.28) is based on the assumption of linear variation of $\bar{\phi}_{0}$ over each free surface element under the conditions of zero atmospheric pressure, zero velocity potential at the upstream inflow surface, and zero outward normal derivative of the velocity potential for a given free surface profile. The value $\bar{\phi}_{0 d}$ in each iteration cycle is also obtained from Equation (3.28) and the corresponding free surface location. 
The dashed lines shown in Table 3.1 mean that the data do not change or there are no applicable data. From Columns 2-5, the shapes of the dam and the free surface for the initial guess and the result at the 100th iteration cycle are illustrated in Figure 3.4. The dam shape bends to the right and carries the $\mathrm{x}$-coordinate of the crest to $\bar{x}_{0}=0.5279$. The overflow depth at the crest is 0.0811 , which is $16 \%$ of the height of the dam $(0.5036)$ and is less than the maximum overflow (40\%) suggested by Bridgestone (1991). This overflow depth is $82 \%$ of the overflow head (far upstream water depth minus the height of the dam), which is higher than the $70 \%$ that Alwan (1988) proposed.

Column 6 in Table 3.1 is for the velocity potential, which is used to generate the flow velocity on the two streamlines (the free surface and the dam/bed combination) in Column 8. From Columns 5 and 8 (the terms regarding the elevation head and the velocity head), Bernoulli values can be calculated at the free surface nodes (Column 9), with the target value of 0.6060 . There are 6 nodes with errors over $3 \%$ on the Bernoulli evaluation, as pointed out in Figure 3.5. It is found that the nodes right over the crest of the dam or around the downstream sharp comer on the free surface yield the larger Bernoulli errors.

The outward normal derivatives of the velocity potential found in Column 7 for the inflow and outflow fictitious boundaries are employed to calculate the flow rate. At Nodes 2 and 52, the $\bar{\phi}_{0, \bar{n}}$ is for the derivative on the side approaching the nodes, while at Nodes 42 and 92 , the $\bar{\phi}_{0, \bar{n}}$ is for the derivative on the side leaving the nodes (clockwise). A linear variation for $\bar{\phi}_{0, \bar{n}}$ is used in the elements and the average of the flow rates obtained at the inflow and the outflow surfaces is taken as the flow rate for the system, which is 0.0368 in the example. For the height of the dam and the overflow water depth in this result, the formula from Bridgestone (Bridgestone, 1991) gives 0.0335 for the flow rate in the case of no downstream water (for the dams with fins). The difference is insignificant. 
The pressure distribution on the dam (from Equation (3.27)) is displayed in Figure 3.6, as well as in Table 3.1, Column 10. The maximum pressure appears at the anchored points (stagnation points) and some negative pressure exists beyond the crest of the dam.

An error function is introduced for the free surface:

$\varepsilon_{f}=\sum_{i=52}^{91} \Delta \bar{s}_{i}\left(\bar{e}_{0}-\frac{\bar{y}_{0 i}+\bar{y}_{0 ; i+1}}{2}-\frac{1}{2} \bar{\phi}_{0 i, \bar{s}}^{2}\right)^{2}$

where $\bar{\phi}_{0 i, s}$ is the velocity for Element $i$ (a linear element has a uniform velocity) and other variables are defined as in Equation (3.28). The error function has the value of 0.0010 at the 100 th iteration cycle (Figure 3.7).

Figure 3.8 shows the variation of velocity potential at the downstream outflow surface, $\bar{\phi}_{0 d}$, with time. Its value is 7.3700 at Cycle 100. The maximum displacement in the same cycle among the nodes on the dam surface is 0.0027 . The location of the critical point stays at either Node 57 or Node $58\left(\bar{x}_{0}=0\right.$ or $\bar{x}_{0}=0.25$, right before the crest of the dam). If the iteration process becomes divergent, it is very often found here that the critical point shifts to a downstream region (after the crest). If the momentum theorem is applied, the sum of the forces in the $\mathrm{x}$-direction is 0.0520 and the net flow rate of momentum through the surfaces of the domain is 0.03745 . This error may result from the fact that $\bar{\phi}_{0, \bar{n}}$ is quite different at Nodes $2,92,42$, and 52 from the values at the rest of the inflow and outflow boundary nodes. The rest is pretty uniform (see Table 3.1) either upstream or downstream. 
Table 3.1 Result at Iteration Cycle 100 (Part 1 of 2)

\begin{tabular}{|c|c|c|c|c|c|c|c|c|c|}
\hline \multirow[b]{2}{*}{$\begin{array}{l}\text { Node } \\
\text { No. }\end{array}$} & \multicolumn{2}{|c|}{ Initial Guess } & \multicolumn{7}{|c|}{ Result at Iteration Cycle 100} \\
\hline & $\bar{x}_{0}$ & $\bar{y}_{0}$ & $\bar{x}_{0}$ & $\bar{y}_{0}$ & $\bar{\phi}_{0}$ & $\bar{\phi}_{0 . \bar{n}}$ & $\bar{\phi}_{0 . \bar{s}}$ & $\begin{array}{l}\text { Bernoulli on } \\
\text { F. S. }\end{array}$ & $\begin{array}{l}\text { Pressure on } \\
\text { Dam }\end{array}$ \\
\hline 2 & 7.0000 & 0.0000 & - & - & 7.3700 & 0.5490 & - & - & - \\
\hline 3 & 1.9000 & 0.0000 & - & - & 1.9100 & 0.0000 & 1.0700 & - & - \\
\hline 4 & 1.8000 & 0.0000 & - & - & 1.8000 & 0.0000 & 1.0900 & - & - \\
\hline 5 & 1.7000 & 0.0000 & - & - & 1.6900 & 0.0000 & 1.0800 & - & - \\
\hline 6 & 1.6000 & 0.0000 & - & - & 1.5900 & 0.0000 & 1.0700 & - & - \\
\hline 7 & 1.5000 & 0.0000 & - & - & 1.4800 & 0.0000 & 1.0500 & - & - \\
\hline 8 & 1.4000 & 0.0000 & - & - & 1.3800 & 0.0000 & 1.0700 & - & - \\
\hline 9 & 1.3000 & 0.0000 & - & - & 1.2700 & 0.0000 & 1.0800 & - & - \\
\hline 10 & 1.2000 & 0.0000 & - & - & 1.1600 & 0.0000 & 1.0700 & - & - \\
\hline 11 & 1.1000 & 0.0000 & - & - & 1.0500 & 0.0000 & 1.0600 & - & - \\
\hline 12 & 1.0000 & 0.0000 & 1.0000 & 0.0000 & 0.9870 & 0.0000 & 0.0000 & - & 0.6060 \\
\hline 13 & 0.9940 & 0.0782 & 0.9995 & 0.0785 & 0.9440 & 0.0000 & 0.5540 & - & 0.3740 \\
\hline 14 & 0.9760 & 0.1550 & 0.9896 & 0.1563 & 0.8680 & 0.0000 & 0.9860 & - & -0.0364 \\
\hline 15 & 0.9460 & 0.2270 & 0.9667 & 0.2314 & 0.7950 & 0.0000 & 0.9540 & - & -0.0805 \\
\hline 16 & 0.9050 & 0.2940 & 0.9303 & 0.3008 & 0.7290 & 0.0000 & 0.8640 & - & -0.0680 \\
\hline 17 & 0.8540 & 0.3540 & 0.8818 & 0.3625 & 0.6700 & 0.0000 & 0.7620 & - & -0.0468 \\
\hline 18 & 0.7940 & 0.4050 & 0.8230 & 0.4144 & 0.6200 & 0.0000 & 0.6460 & - & -0.0171 \\
\hline 19 & 0.7270 & 0.4460 & 0.7560 & 0.4552 & 0.5790 & 0.0000 & 0.5180 & - & 0.0166 \\
\hline 20 & 0.6550 & 0.4760 & 0.6831 & 0.4840 & 0.5460 & 0.0000 & 0.4180 & - & 0.0346 \\
\hline 21 & 0.5780 & 0.4940 & 0.6063 & 0.5002 & 0.5160 & 0.0000 & 0.3920 & - & 0.0290 \\
\hline 22 & 0.5000 & 0.5000 & 0.5279 & 0.5036 & 0.4850 & 0.0000 & 0.3940 & - & 0.0248 \\
\hline 23 & 0.4220 & 0.4940 & 0.4501 & 0.4942 & 0.4560 & 0.0000 & 0.3670 & - & 0.0445 \\
\hline 24 & 0.3460 & 0.4760 & 0.3747 & 0.4724 & 0.4310 & 0.0000 & 0.3230 & - & 0.0814 \\
\hline 25 & 0.2730 & 0.4460 & 0.3036 & 0.4392 & 0.4100 & 0.0000 & 0.2680 & - & 0.1309 \\
\hline 26 & 0.2060 & 0.4050 & 0.2382 & 0.3959 & 0.3940 & 0.0000 & 0.2100 & - & 0.1881 \\
\hline 27 & 0.1460 & 0.3540 & 0.1796 & 0.3437 & 0.3810 & 0.0000 & 0.1610 & - & 0.2493 \\
\hline 28 & 0.0955 & 0.2940 & 0.1284 & 0.2842 & 0.3720 & 0.0000 & 0.1220 & - & 0.3144 \\
\hline 29 & 0.0545 & 0.2270 & 0.0849 & 0.2188 & 0.3650 & 0.0000 & 0.0902 & - & 0.3831 \\
\hline 30 & 0.0245 & 0.1550 & 0.0493 & 0.1489 & 0.3600 & 0.0000 & 0.0633 & - & 0.4551 \\
\hline 31 & 0.0062 & 0.0782 & 0.0211 & 0.0756 & 0.3560 & 0.0000 & 0.0398 & - & 0.5296 \\
\hline 32 & 0.0000 & 0.0000 & 0.0000 & 0.0000 & 0.3560 & 0.0000 & 0.0000 & - & 0.6060 \\
\hline 33 & -0.1000 & 0.0000 & - & -1 & 0.3540 & 0.0000 & 0.0197 & - & - \\
\hline 34. & $-0 . \overline{2000}$ & 0.0000 & - & - & 0.3500 & 0.0000 & 0.0357 & - & - \\
\hline 35 & -0.3000 & 0.0000 & - & - & 0.3460 & 0.0000 & 0.0448 & - & - \\
\hline 36 & -0.4000 & 0.0000 & - & - & 0.3410 & 0.0000 & 0.0501 & - & - \\
\hline 37 & -0.5000 & 0.0000 & - & - & 0.3350 & 0.0000 & 0.0533 & - & - \\
\hline 38 & -0.6000 & 0.0000 & - & - & 0.3300 & 0.0000 & 0.0552 & - & - \\
\hline 39 & -0.7000 & 0.0000 & - & - & 0.3240 & 0.0000 & 0.0564 & - & - \\
\hline 40 & -0.8000 & 0.0000 & - & - & 0.3180 & 0.0000 & 0.0572 & - & - \\
\hline 41 & -0.9000 & 0.0000 & - & - & 0.3130 & 0.0000 & 0.0578 & - & - \\
\hline 42 & -6.0000 & 0.0000 & - & 0.0000 & 0.0000 & -0.0904 & - & $\cdot$ & - \\
\hline 43 & -6.0000 & 0.0600 & - & 0.0608 & 0.0000 & -0.0634 & - & - & - \\
\hline 44 & -6.0000 & 0.1200 & - & 0.1220 & 0.0000 & -0.0663 & - & $=$ & - \\
\hline 45 & -6.0000 & 0.1800 & - & 0.1830 & 0.0000 & -0.0652 & - & - & - \\
\hline 46 & -6.0000 & 0.2400 & - & 0.2430 & 0.0000 & -0.0650 & - & - & - \\
\hline 47 & -6.0000 & 0.3000 & - & 0.3040 & 0.0000 & -0.0649 & $\because$ & - & - \\
\hline 48 & -6.0000 & 0.3600 & - & 0.3650 & 0.0000 & -0.0649 & - & - & - \\
\hline 49 & -6.0000 & 0.4200 & - & 0.4260 & 0.0000 & -0.0649 & - & - & - \\
\hline 50 & -6.0000 & 0.4800 & - & 0.4870 & 0.0000 & -0.0656 & - & - & - \\
\hline 511 & -6.0000 & 0.5400 & - & 0.5470 & 0.0000 & -0.0635 & - & - & - \\
\hline
\end{tabular}


Table 3.1 Result at Iteration Cycle 100 (Part 2 of 2)

\begin{tabular}{|c|c|c|c|c|c|c|c|c|c|}
\hline \multirow[b]{2}{*}{$\begin{array}{l}\text { Node } \\
\text { No. }\end{array}$} & \multicolumn{2}{|c|}{ Initial Guess } & \multicolumn{7}{|c|}{ Result at Iteration Cycle 100} \\
\hline & $\bar{x}_{0}$ & $\bar{y}_{0}$ & $\bar{x}_{0}$ & $\bar{y}_{0}$ & $\bar{\phi}_{0}$ & $\bar{\phi}_{0, \bar{n}}$ & $\bar{\phi}_{0, \bar{s}}$ & $\begin{array}{l}\text { Benoulli on } \\
\text { F. S. }\end{array}$ & $\begin{array}{l}\text { Pressure on } \\
\text { Dam }\end{array}$ \\
\hline 52 & -6.0000 & 0.6000 & - & 0.6020 & 0.0000 & -0.0824 & - & - & - \\
\hline 53 & -3.0000 & 0.5940 & - & 0.6020 & 0.1860 & 0.0000 & 0.0620 & 0.6039 & - \\
\hline 54 & -1.0000 & 0.5820 & - & 0.6020 & 0.3060 & 0.0000 & 0.0600 & 0.6038 & $=$ \\
\hline 55 & -0.5000 & 0.5790 & - & 0.6020 & 0.3360 & 0.0000 & 0.0600 & 0.6038 & $=$ \\
\hline 56 & -0.2500 & 0.5770 & - & 0.6020 & 0.3520 & 0.0000 & 0.0640 & 0.6040 & - \\
\hline 57 & 0.0000 & 0.5740 & - & 0.5970 & 0.3710 & 0.0000 & 0.1360 & 0.6062 & - \\
\hline 58 & 0.2500 & 0.5720 & - & 0.5920 & 0.4060 & 0.0000 & 0.2730 & 0.6293 & - \\
\hline 59 & 0.5000 & 0.5700 & - & 0.5880 & 0.4740 & 0.0000 & 0.3520 & 0.6500 & - \\
\hline 60 & 0.5420 & 0.5680 & - & 0.5830 & 0.4890 & 0.0000 & 0.3640 & 0.6492 & - \\
\hline 61 & 0.6040 & 0.5580 & - & 0.5790 & 0.5110 & 0.0000 & 0.3420 & 0.6375 & - \\
\hline 62 & 0.6670 & 0.5390 & $=$ & 0.5720 & 0.5330 & 0.0000 & 0.2980 & 06164 & - \\
\hline 63 & 0.7080 & 0.5220 & - & 0.5650 & 0.5450 & 0.0000 & 0.2700 & 0.6015 & - \\
\hline 64 & 0.7290 & 0.5110 & - & 0.5550 & 0.5520 & 0.0000 & 0.3080 & 0.6024 & - \\
\hline 65 & 0.7500 & 0.5000 & - & 0.5420 & 0.5590 & 0.0000 & 0.3270 & 0.5955 & - \\
\hline 66 & 0.7710 & 0.4870 & - & 0.5290 & 0.5670 & 0.0000 & 0.3640 & 0.5952 & - \\
\hline 67 & 0.7920 & 0.4720 & - & 0.5100 & 0.5780 & 0.0000 & 0.4120 & 0.5949 & - \\
\hline 68 & 0.8130 & 0.4560 & - & 0.4920 & 0.5890 & 0.0000 & 0.4600 & 0.5978 & - \\
\hline 69 & 0.8330 & 0.4380 & - & 0.4710 & 0.6030 & 0.0000 & 0.5080 & 0.6000 & - \\
\hline 70 & 0.8540 & 0.4180 & - & 0.4510 & 0.6170 & 0.0000 & 0.5490 & 0.6017 & - \\
\hline 71 & 0.8750 & 0.3960 & - & 0.4290 & 0.6340 & 0.0000 & 0.5920 & 0.6042 & - \\
\hline 72 & 0.8960 & 0.3700 & - & 0.4070 & 0.6520 & 0.0000 & 0.6340 & 0.6080 & - \\
\hline 73 & 0.9170 & 0.3410 & - & 0.3800 & 0.6730 & 0.0000 & 0.6730 & 0.6065 & - \\
\hline 74 & 0.9380 & 0.3060 & - & 0.3540 & 0.6960 & 0.0000 & 0.7210 & 0.6139 & - \\
\hline 75 & 0.9580 & 0.2640 & - & 0.3210 & 0.7240 & 0.0000 & 0.7610 & 0.6106 & - \\
\hline 76 & 0.9790 & 0.2060 & - & 0.2860 & 0.7550 & 0.0000 & 0.8210 & 0.6230 & - \\
\hline 77 & 1.0000 & 0.1350 & - & 0.2350 & 0.8000 & 0.0000 & 0.8580 & 0.6031 & - \\
\hline 78 & 1.0100 & 0.0631 & - & 0.2010 & 0.8310 & 0.0000 & 0.9200 & 0.6242 & - \\
\hline 79 & 1.0300 & 0.0631 & - & 0.1400 & 0.8890 & 0.0000 & 0.9540 & 0.5951 & - \\
\hline 80 & 1.0400 & 0.0631 & - & 0.0571 & 0.9680 & 0.0000 & 1.0500 & 0.6084 & - \\
\hline 81 & 1.0500 & 0.0631 & - & 0.0308 & 0.9990 & 0.0000 & 1.0900 & 0.6249 & - \\
\hline 82 & 1.1000 & 0.0631 & - & 0.0315 & 1.0500 & 0.0000 & 1.0500 & 0.5828 & - \\
\hline 83 & 1.1500 & 0.0631 & - & 0.0285 & 1.1100 & 0.0000 & 1.0900 & 0.6226 & - \\
\hline 84 & 1.2000 & 0.0631 & - & 0.0282 & 1.1600 & 0.0000 & 1.0600 & 0.5900 & - \\
\hline 85 & 1.2500 & 0.0631 & - & 0.0292 & 1.2100 & 0.0000 & 1.0800 & 0.6124 & - \\
\hline 86 & 1.3000 & 0.0631 & - & 0.0269 & 1.2700 & 0.0000 & 1.0700 & 0.5994 & - \\
\hline 87 & 1.3500 & 0.0631 & - & 0.0295 & 1.3200 & 0.0000 & 1.0800 & 0.6127 & - \\
\hline 88 & 1.4000 & 0.0631 & - & 0.0260 & 1.3800 & 0.0000 & 1.0700 & 0.5985 & - \\
\hline 89 & 1.5000 & 0.0631 & - & 0.0304 & 1.4800 & 0.0000 & 1.0700 & 0.6029 & - \\
\hline 90 & 2.0000 & 0.0631 & - & 0.0193 & 2.0200 & 0.0000 & 1.0800 & 0.6025 & - \\
\hline 91 & 4.0000 & 0.0631 & - & 0.0342 & 4.1700 & 0.0000 & 1.0700 & 0.6067 & - \\
\hline 92 & 7.0000 & 0.0631 & - & 0.0342 & 7.3700 & 0.6330 & - & - & - \\
\hline 93 & 7.0000 & 0.0568 & - & 0.0307 & 7.3700 & 1.0300 & - & - & - \\
\hline 94 & 7.0000 & 0.0505 & - & 0.0273 & 7.3700 & 0.9890 & - & - & - \\
\hline 95 & 7.0000 & 0.0442 & - & 0.0239 & 7.3700 & 1.0000 & - & - & - \\
\hline 96 & 7.0000 & 0.0378 & - & 0.0205 & 7.3700 & 1.0100 & - & - & - \\
\hline 97 & 7.0000 & 0.0315 & - & 0.0171 & 7.3700 & 1.0100 & - & - & - \\
\hline 98 & 7.0000 & 0.0252 & - & 0.0137 & 7.3700 & 1.0000 & - & - & - \\
\hline 99 & 7.0000 & 0.0189 & - & 0.0102 & 7.3700 & 1.0000 & - & - & - \\
\hline 100 & 7.0000 & 0.0126 & - & 0.0068 & 7.3700 & 0.9810 & - & - & - \\
\hline 101 & 7.0000 & 0.0063 & - & 0.0034 & 7.3700 & 1.0300 & - & - & - \\
\hline
\end{tabular}




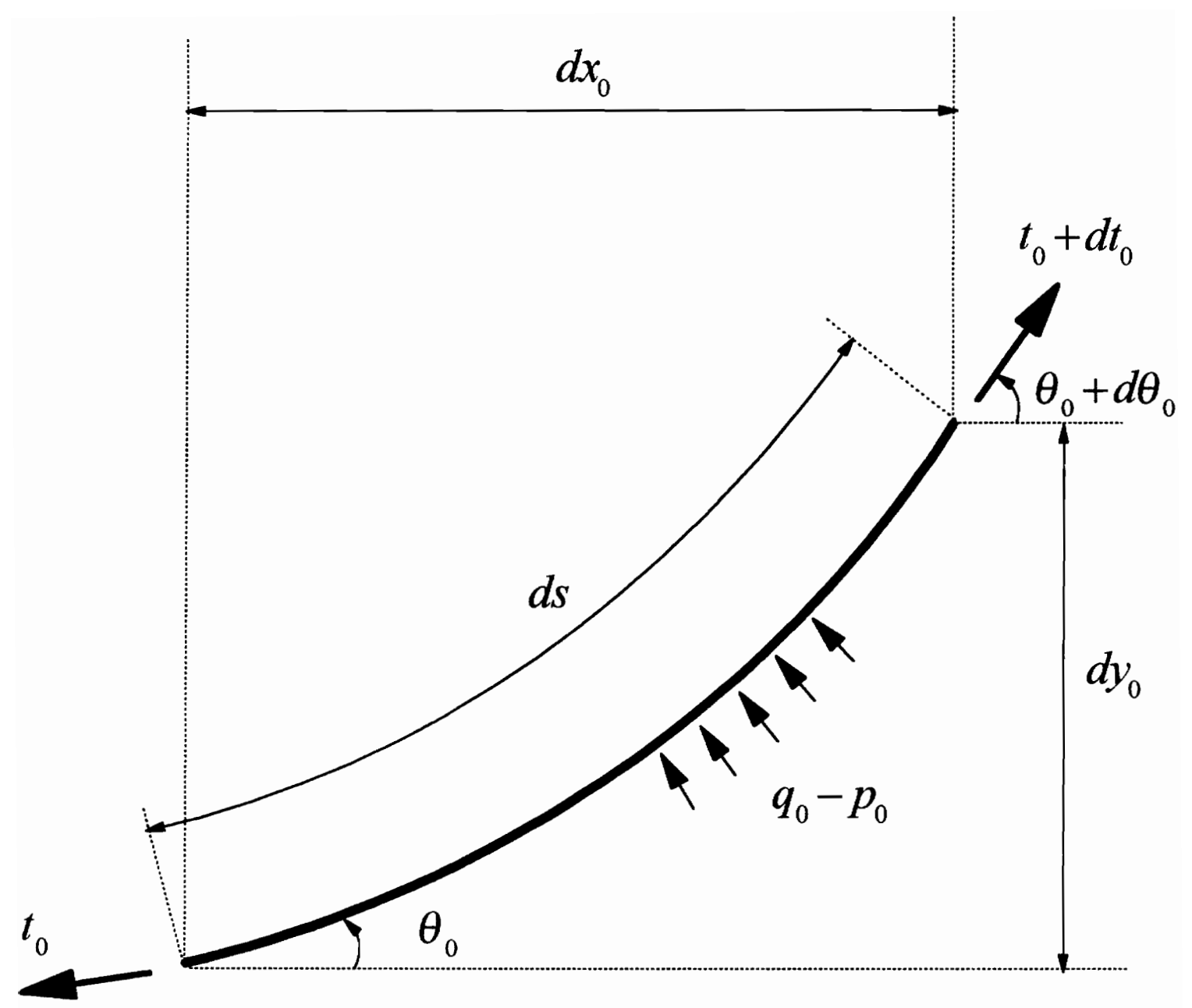

Figure 3.1 A Membrane Element in Equilibrium 


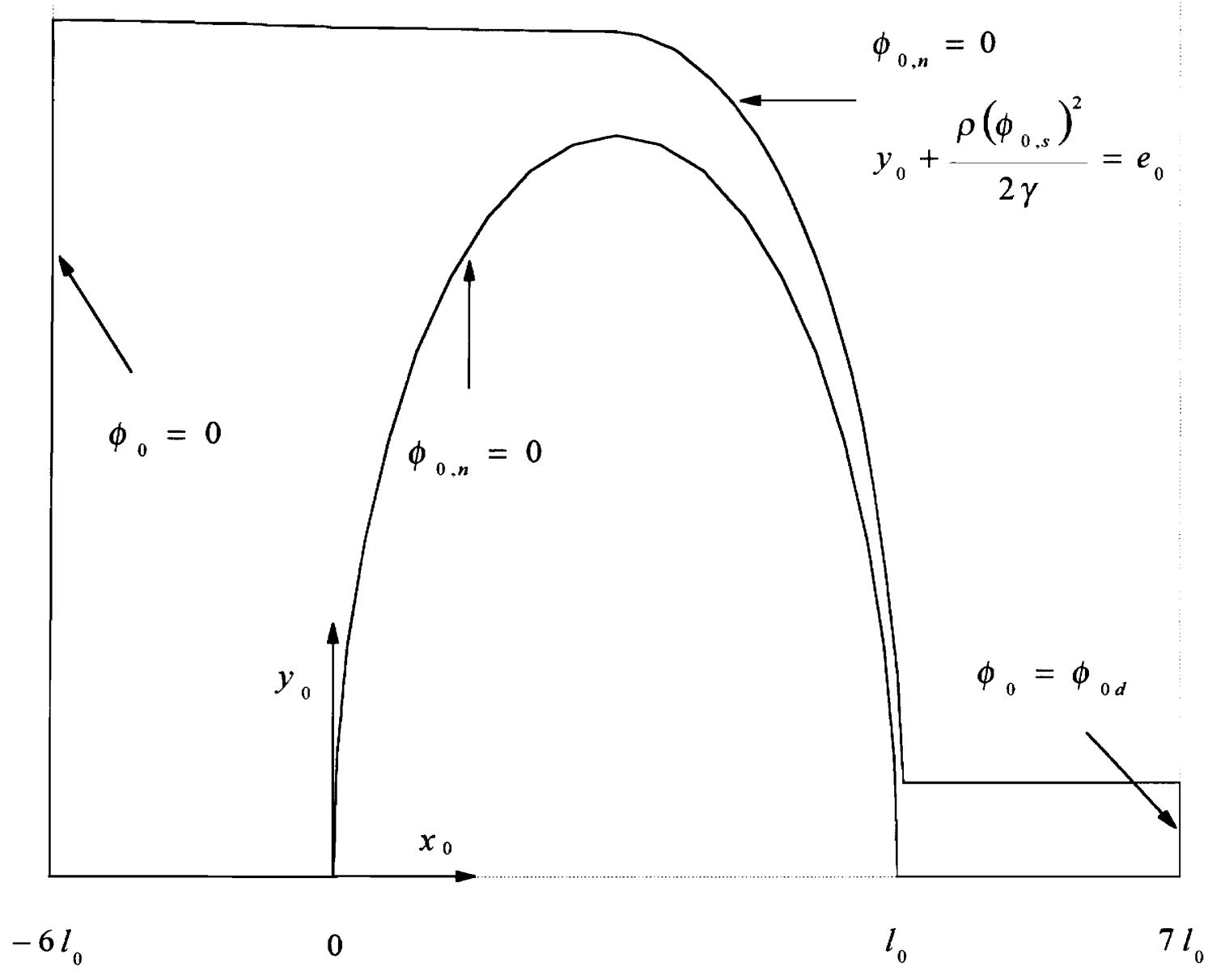

Figure 3.2 The Flow Domain 

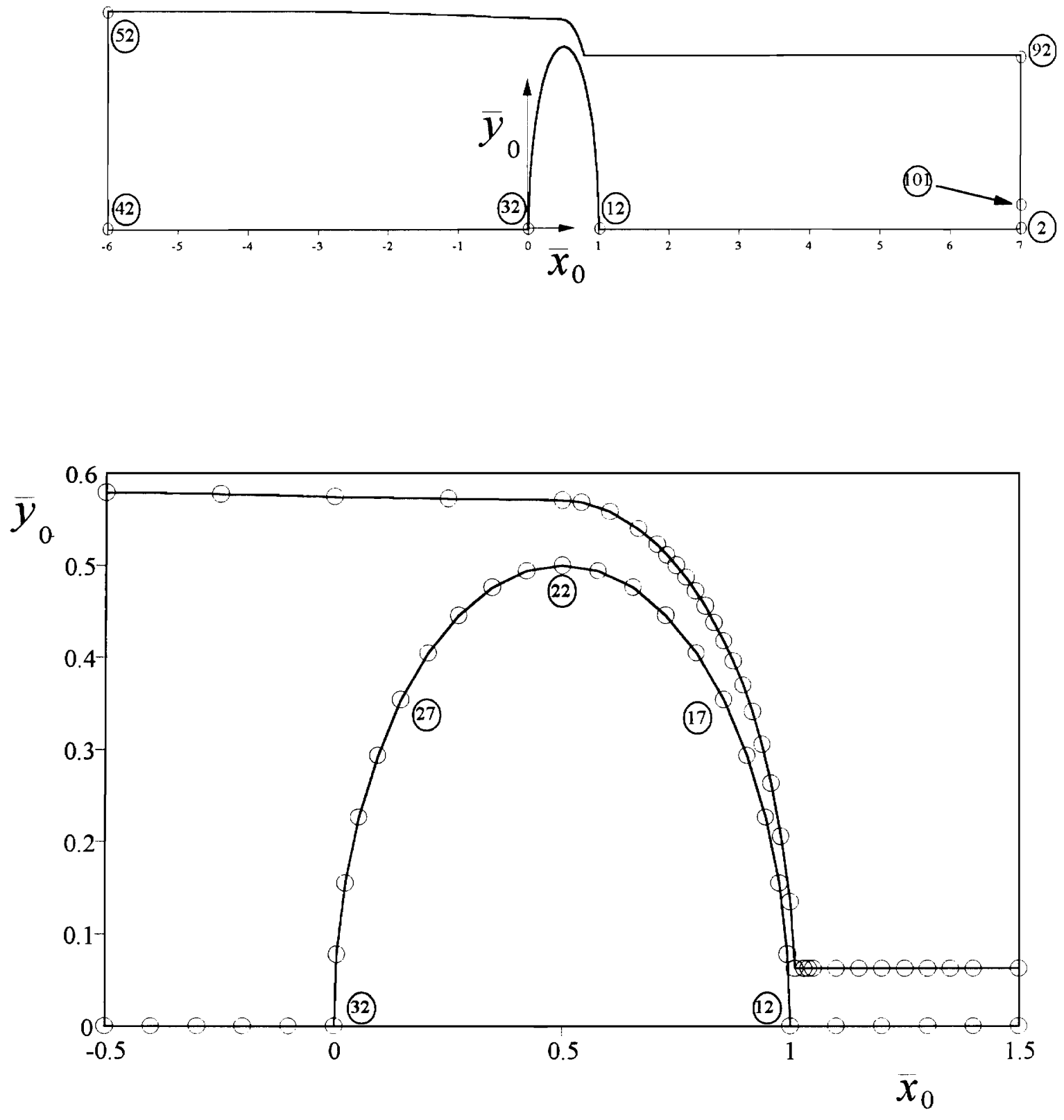

Figure 3.3 BEM Node Setup 


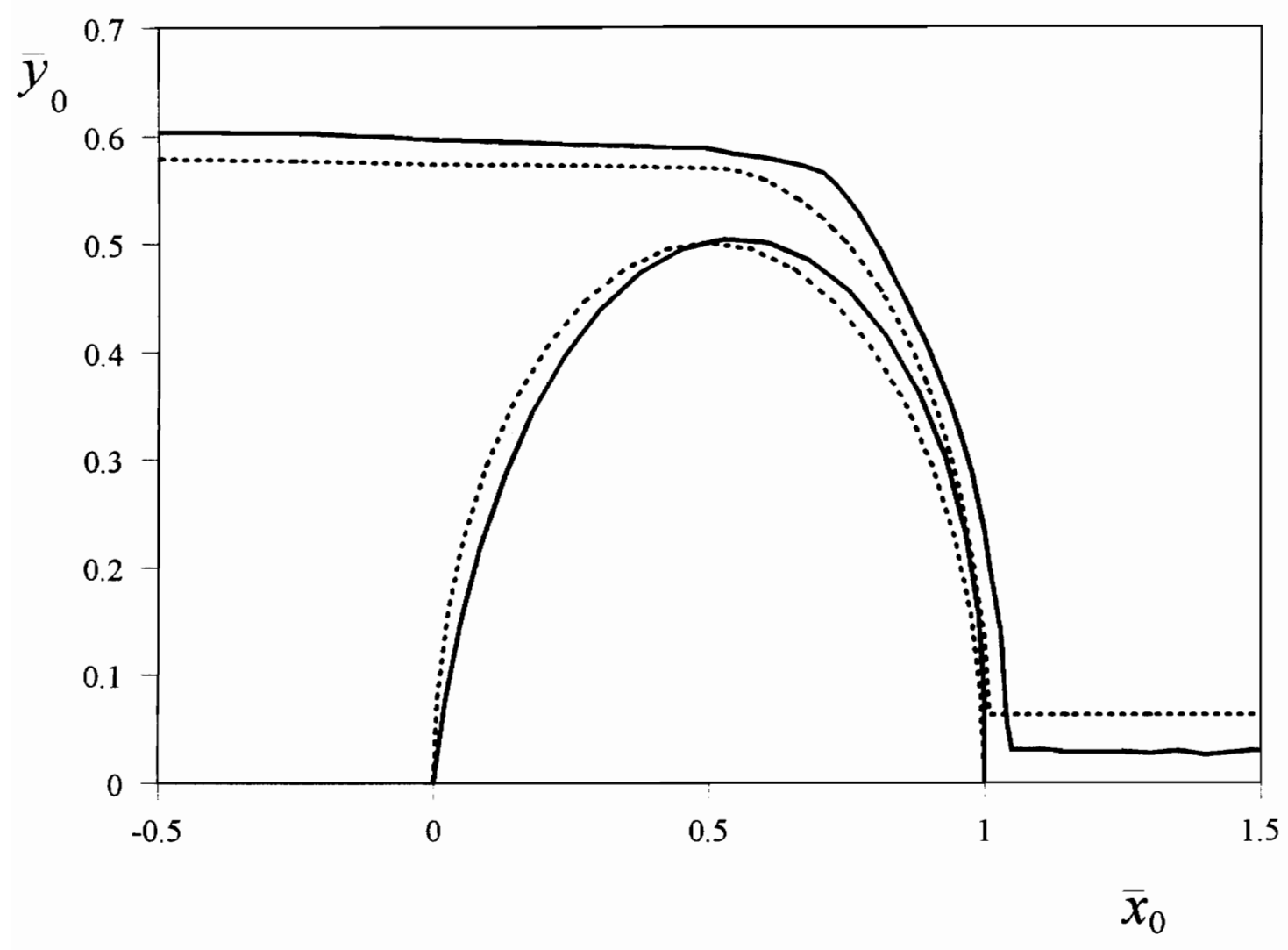

Initial Guess

Result at Iteration Cycle 100

Figure 3.4 Result at Iteration Cycle 100 


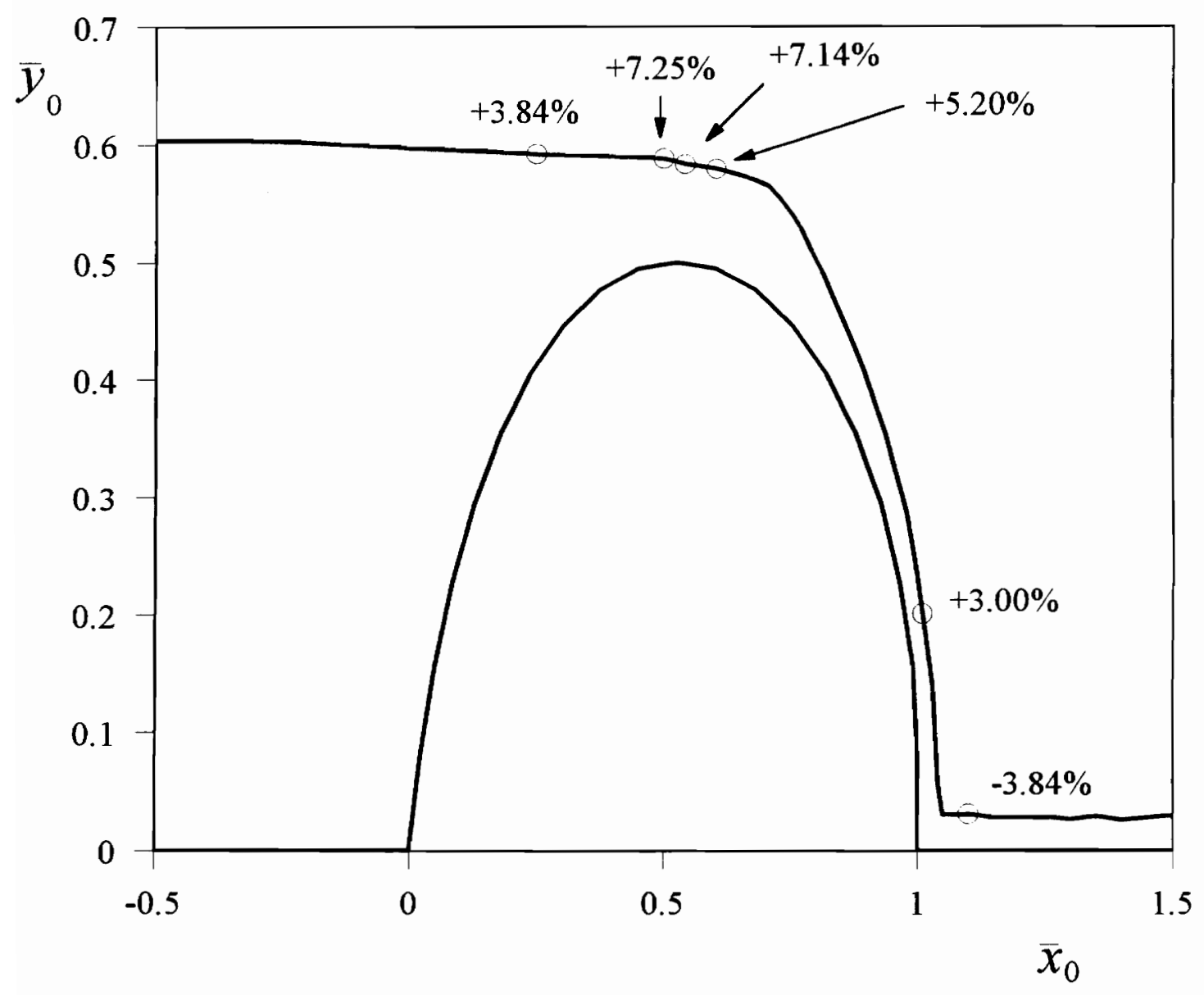

Figure 3.5 Nodes on Free Surface with Bernoulli Error over 3\% 


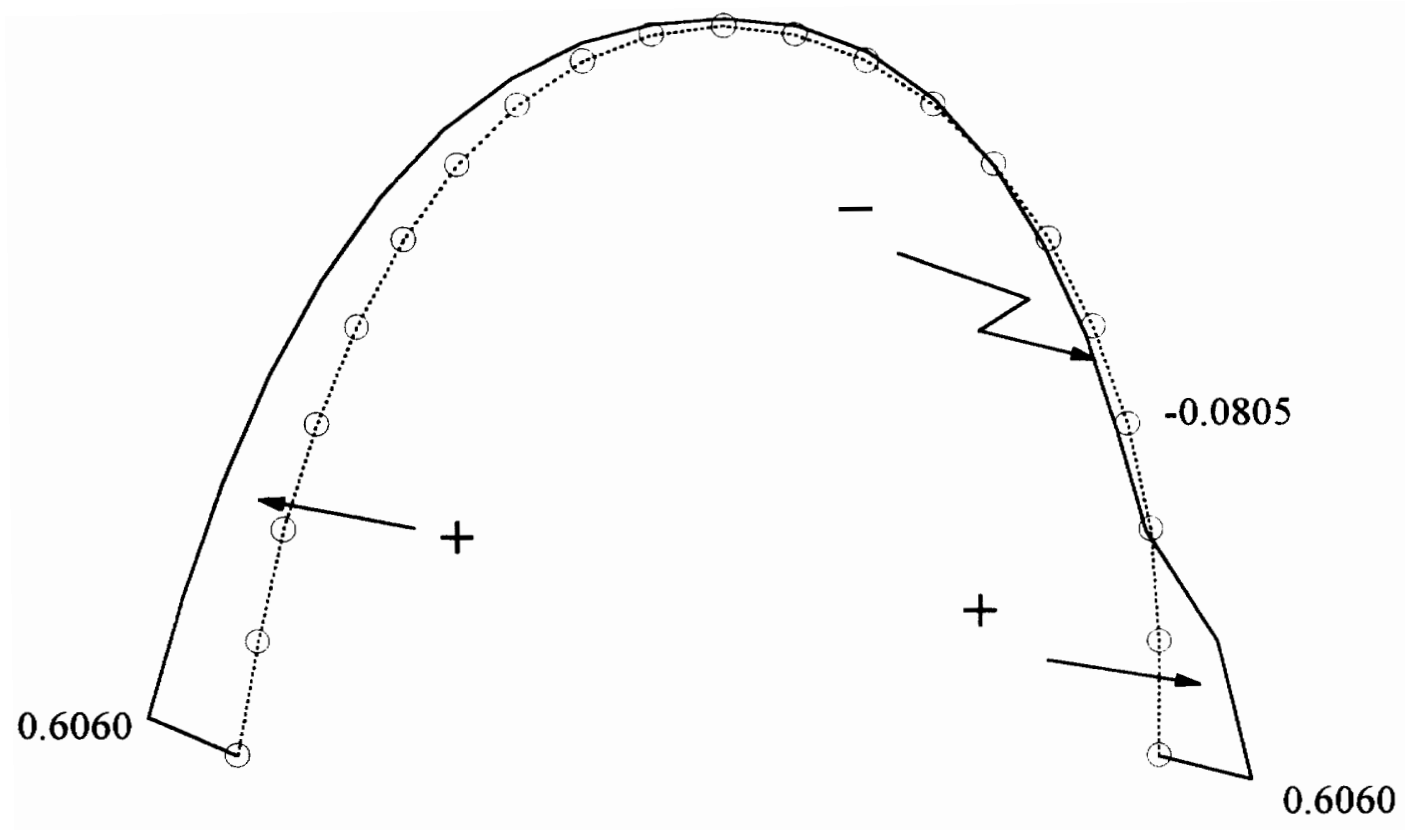

Figure 3.6 Pressure Distribution on Dam Surface 


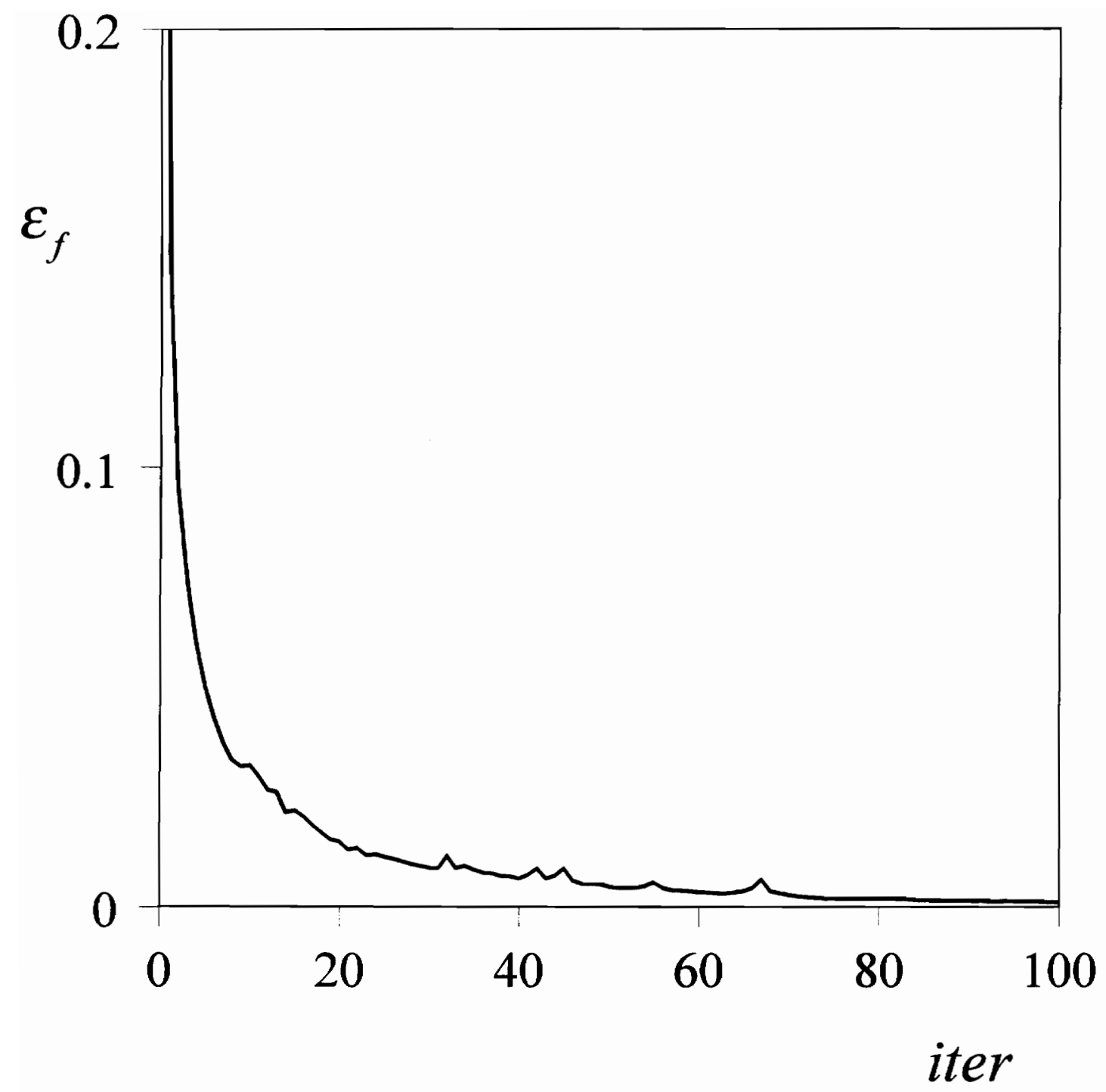

Figure 3.7 Error Function on the Free Surface with Iteration Cycle 


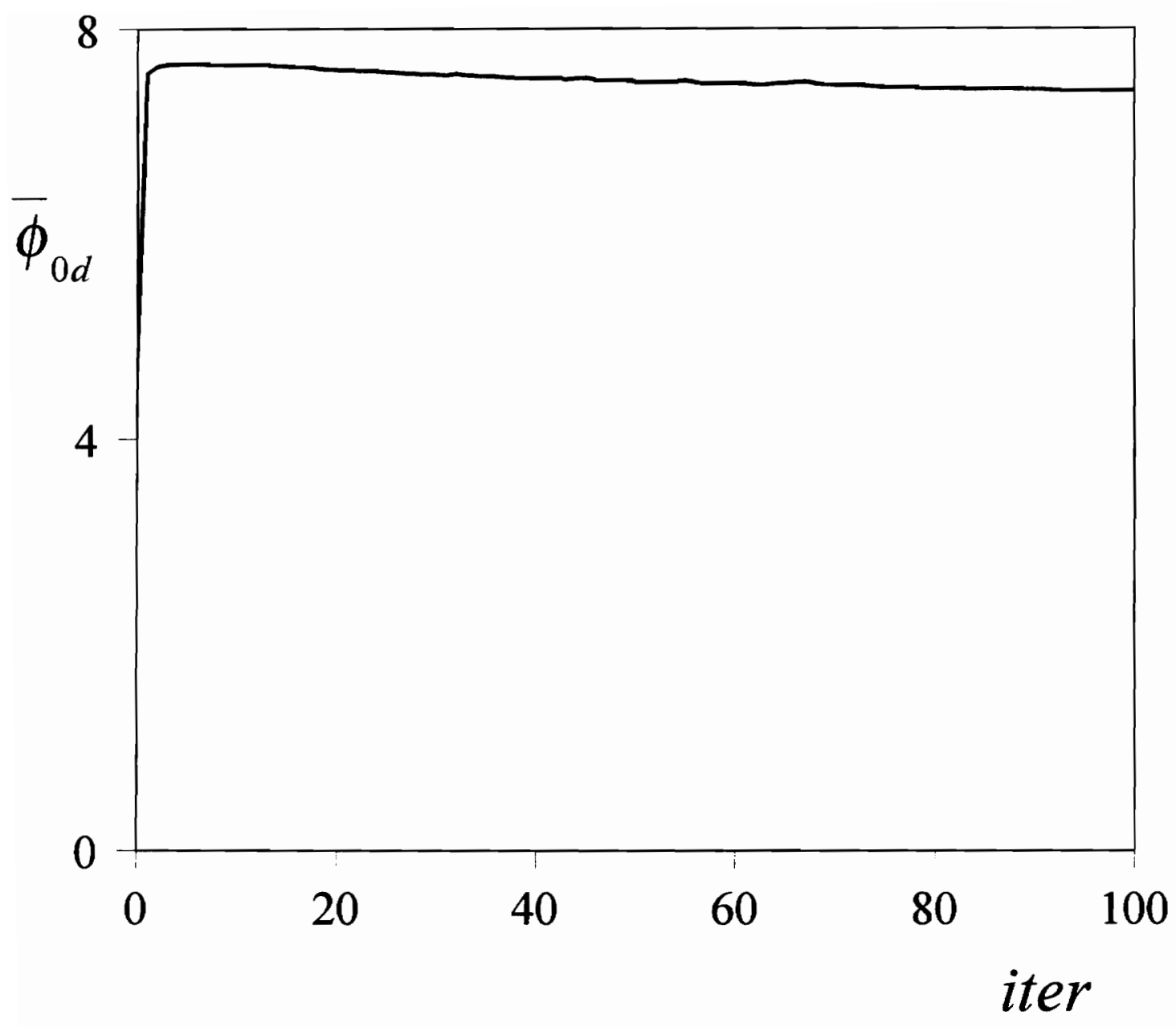

Figure 3.8 Velocity Potential at Downstream Outflow Surface with Iteration Cycle 


\section{Chapter 4}

\section{DYNAMIC ANALYSIS}

\subsection{INTRODUCTION TO DYNAMIC ANALYSIS}

For an inflatable dam under an overflow condition, the static equilibrium shape of the dam, as well as the external pressure distribution over the dam surface and the location of the free surface for the overflow found in Chapter 3, afford the fundamentals for a dynamic analysis.

Small vibration about the equilibrium state is assumed and the equations of motion based on membrane theory prevail in the dynamic analysis. Besides, the membrane of the dam is inextensible and the internal inflated pressure of dam is constant. The overflow is governed by Laplace's equation with gravitational wave propagation neglected and the domain of the flow unchanged as the dam performs vibrations with or without structural damping. An eigenvalue system is established by combining the boundary element method applied on the fluid domain and the membrane equations of motion employed for the displacements of the dam. The vibration modes and vibration frequencies of this eigenvalue system provide the information on how the dam vibrates and if the oscillation is associated with stability, divergence, or flutter. 


\subsection{EQUATIONS OF MOTION FOR THE MEMBRANE}

The shape of the inflatable dam in this two-dimensional analysis is treated as a series of membrane elements with unit depth (in the direction into the paper). One of these membrane elements for the dam in vibration is shown in Figure 4.1. By membrane theory (Firt, 1983) with structural damping, the equations of motion in the tangential and outward normal directions for the membrane element can be expressed as

$\mu \nu{ }_{\tau \tau} d s+\beta v,{ }_{\tau} d s=\left(t+t,{ }_{s} d s\right) \cos d \theta-t$

$\mu w{ }_{\tau \tau} d s+\beta w,{ }_{\tau} d s=(2 t+t, d s) \sin \frac{d \theta}{2}+q d s$

where $\mu$ is the mass per unit area of the membrane, which is a constant; $\beta$ is the structural damping coefficient per unit depth and has units of $\left(\frac{\text { mass }}{\text { time } \cdot \text { length }^{2}}\right) ; \tau$ is the time; $s$ is the curved coordinate; $v(s, \tau)$ and $w(s, \tau)$ are the tangential and outward normal displacements of the membrane, respectively, about the equilibrium state, which are assumed to be small; $t(s, \tau)$ is the total tension per unit depth in the membrane; $\theta(s, \tau)$ is the total slope of the membrane; and $q(s, \tau)$ is the total outward pressure (including internal and external pressure) applied on the membrane, times the unit depth.

In the dynamic analysis, the terms $t, \theta$, and $q$ are 'total' because they are the results of the addition of static solutions and dynamic solutions (caused by the membrane vibration). That is to say

$t=t_{0}+t_{1}$

$\theta=\theta_{0}+\theta_{1}$

$q=q_{0}-\left(p_{0}+p_{1}\right)$

where $t_{0}$ is the tension per unit depth in the membrane in the static analysis, which is uniform along the curved coordinate; $t_{1}(s, \tau)$ is the additional tension caused by the membrane vibration per unit 
depth; $\theta_{0}(s)$ is the slope of the membrane in the static analysis; $\theta_{1}(s, \tau)$ is the additional slope of the membrane caused by the membrane vibration; $q_{0}$ is the internal inflated pressure times the unit depth, which is a constant; $p_{0}(s)$ is the external pressure times the unit depth in the static analysis; and $p_{1}(s, \tau)$ is the additional external pressure caused by the membrane vibration, times the unit depth.

The equations of motion for the membrane element, Equations (4.1) and (4.2), can be simplified since $d \theta$ is assumed to be small and the higher order terms are neglected:

$\mu v,_{\tau \tau}+\beta v_{, \tau}=t_{s}$

$\mu w,_{\tau \tau}+\beta w_{\tau}=t \theta_{, s}+q$

Solving Equation (4.7) for $t$, differentiating $t$ with respect to $s$, substituting $t, s$ back into Equation (4.6), and multiplying Equation (4.6) by $\theta, 2$, we obtain the following equation:

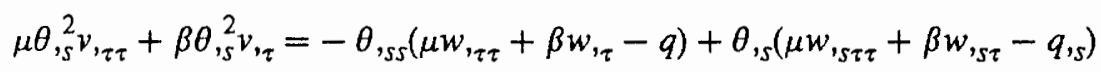

Following Henrych (1981), for small dynamic displacements the term $\theta_{,}$in Equation (4.8) can be approximated by

$\theta, s=\theta_{0, s}+w, s s+\left(\theta_{0, s}\right)^{2} w+\theta_{0, s s} v$

where $\theta_{0, s}$ is the curvature of the dam shape (in the two-dimensional point of view) in the static analysis. By the inextensibility of the membrane, the tangential and the outward normal displacements are related by

$w=v, \theta=\theta,_{s}^{-1} v,{ }_{s} \cong \theta_{0, s}^{-1} v,{ }_{s}$

where the approximation in Equation (4.10) is the result of ignoring the higher order terms in Equation (4.9). 
From the expressions for $\theta_{s}$ and $w$ in Equations (4.9) and (4.10), and the derivatives of them,

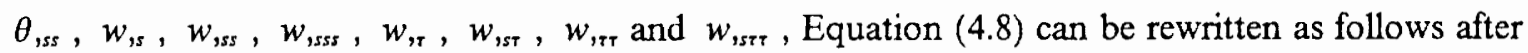
the higher order terms are ignored:

$$
\begin{aligned}
& \mu\left(a_{1}^{2} v_{{ }_{\tau} \tau}+2 a_{1}^{-1} a_{2} v_{{ }_{s \tau \tau}}-v_{s_{s \tau \tau}}\right)+\beta\left(a_{1}^{2} v_{,_{\tau}}+2 a_{1}^{-1} a_{2} v_{s_{s \tau}}-v_{,_{s \tau}}\right)
\end{aligned}
$$

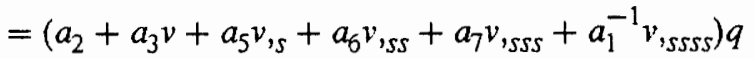

$$
\begin{aligned}
& -\left(a_{1}+a_{2} v+a_{8} v_{s}+a_{9} v_{s s}+a_{1}^{-1} v_{, s s s}\right) q_{, s}
\end{aligned}
$$

in which

$$
\begin{aligned}
& a_{1}=\theta_{0, s} ; \quad a_{2}=a_{1, s} ; \quad a_{3}=a_{2, s} ; \quad a_{4}=a_{3, s} ; \\
& a_{5}=-6 a_{1}^{-4} a_{2}^{3}+6 a_{1}^{-3} a_{2} a_{3}-a_{1}^{-2} a_{4}+2 a_{2} ; \\
& a_{6}=6 a_{1}^{-3} a_{2}^{2}-3 a_{1}^{-2} a_{3}+a_{1} ; \quad a_{7}=-3 a_{1}^{-2} a_{2} ; \\
& a_{8}=2 a_{1}^{-3} a_{2}^{2}-a_{1}^{-2} a_{3}+a_{1} ; \quad a_{9}=-2 a_{1}^{-2} a_{2}
\end{aligned}
$$

In Equation (4.11), the terms $q$ and $q, s$ can be put in a more explicit form by recalling Equations (4.5) and (3.8):

$q=q_{0}-\left(p_{0}+p_{1}\right)$

$\frac{d \theta_{0}}{d s}=\frac{\left(p_{0}-q_{0}\right)}{t_{0}}$

The terms $q$ and $q, s$ thus become

$$
\begin{aligned}
& q=-a_{1} t_{0}-p_{1} \\
& q_{, s}=-a_{2} t_{0}-p_{1, s}
\end{aligned}
$$

where $a_{1}$ and $a_{2}$ are defined in Equation (4.12). From Equations (4.13) and (4.14), assuming that $p_{1}$ is of the same order as $v$, and neglecting the higher order terms, we rewrite Equation (4.11) as 


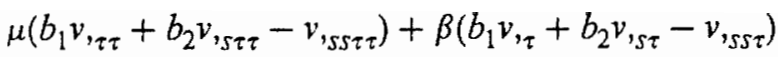

$$
\begin{aligned}
& =t_{0}\left(b_{3} v+b_{4} v_{, s}+b_{5} v_{, s s}+b_{6} v_{s s s}-v_{s_{s s s s}}\right)+b_{7} p_{1}+b_{8} p_{1, s}
\end{aligned}
$$

where

$$
\begin{aligned}
& b_{1}=a_{1}^{2} ; \quad b_{2}=2 a_{1}^{-1} a_{2} ; \quad b_{3}=a_{2}^{2}-a_{1} a_{3} ; \\
& b_{4}=a_{2} a_{8}-a_{1} a_{5}=8 a_{1}^{-3} a_{2}^{3}-7 a_{1}^{-2} a_{2} a_{3}+a_{1}^{-1} a_{4}-a_{1} a_{2} ; \\
& b_{5}=a_{2} a_{9}-a_{1} a_{6}=-8 a_{1}^{-2} a_{2}^{2}+3 a_{1}^{-1} a_{3}-a_{1}^{2} ; \\
& b_{6}=a_{1}^{-1} a_{2}-a_{1} a_{7}=4 a_{1}^{-1} a_{2} ; \quad b_{7}=-a_{2} ; \quad b_{8}=a_{1}
\end{aligned}
$$

In order to nondimensionalize the equation of motion, Equation (4.15), the following set of variables is introduced based on $l_{0}$ (the base width of the dam), $\gamma$ (the specific weight of the fluid in the overflow), and $\mu$ (the mass per unit area of the dam material):

$$
\begin{aligned}
& \bar{s}=\frac{s}{l_{0}} ; \quad \bar{\tau}=\sqrt{\frac{\gamma}{\mu}} \tau ; \quad \bar{\beta}=\sqrt{\frac{1}{\gamma \mu}} \beta ; \\
& \bar{\theta}_{0}=\theta_{0} ; \quad \bar{a}_{1}=l_{0} a_{1} ; \quad \bar{a}_{2}=l_{0}^{2} a_{2} ; \quad \bar{a}_{3}=l_{0}^{3} a_{3} ; \quad \bar{a}_{4}=l_{0}^{4} a_{4} ; \\
& \bar{a}_{5}=l_{0}^{2} a_{5} ; \quad \bar{a}_{6}=l_{0} a_{6} ; \quad \bar{a}_{7}=a_{7} ; \quad \bar{a}_{8}=l_{0} a_{8} ; \quad \bar{a}_{9}=a_{9} ; \\
& \bar{b}_{1}=l_{0}^{2} b_{1} ; \quad \bar{b}_{2}=l_{0} b_{2} ; \quad \bar{b}_{3}=t_{0} b_{3} ; \quad \bar{b}_{4}=l_{0}^{3} b_{4} ; \\
& \bar{b}_{5}=l_{0}^{2} b_{5} ; \quad \bar{b}_{6}=l_{0} b_{6} ; \quad \bar{b}_{7}=l_{0}^{2} b_{7} ; \quad \bar{b}_{8}=l_{0} b_{8} ; \\
& \bar{t}_{0}=\frac{t_{0}}{\gamma l_{0}^{2}} ; \quad \bar{p}_{1}=\frac{p_{1}}{\gamma l_{0}} ; \quad \bar{p}_{1, \bar{s}}=\frac{p_{1, s}}{\gamma} ; \\
& \bar{v}=\frac{v}{l_{0}} ; \quad \bar{v}_{\bar{s}}=v_{, s} ; \quad \bar{v}_{, \overline{s s}}=l_{0} v_{, s s} ; \quad \bar{v}_{\bar{s} \bar{s} \bar{s}}=l_{0}^{2} v_{, s s s} ; \quad \bar{v}_{\bar{s} \bar{s} \bar{s} s}=l_{0}^{3} v_{, s s s s} ;
\end{aligned}
$$


$\bar{v}_{, \bar{\tau}}=\sqrt{\frac{\mu}{\gamma}} \frac{1}{l_{0}} v_{,_{\tau}} ; \quad \bar{v}_{\overline{s \tau}_{\overline{s \tau}}}=\sqrt{\frac{\mu}{\gamma}} v_{,_{s \tau}} ; \quad \bar{v}_{,_{\bar{s}} \overline{s \tau}}=\sqrt{\frac{\mu}{\gamma}} l_{0} v_{s_{s s \tau}} ;$

$\bar{v}, \overline{\tau \tau}=\frac{\mu}{\gamma l_{0}} v_{, \tau \tau} ; \quad \bar{v}_{, \overline{s \tau \tau}}=\frac{\mu}{\gamma} v_{s_{s \tau \tau}} ; \quad \bar{v}_{, \bar{s} \overline{s \tau \tau}}=\frac{\mu}{\gamma} l_{0} v_{s s \tau \tau}$

After nondimensionalization by Equation (4.17), Equation (4.15) turns into

$$
\begin{aligned}
& \bar{b}_{1} \bar{v}, \overline{\tau \tau}+\bar{b}_{2} \bar{v}, \overline{s \tau \tau}-\bar{v}, \bar{s} \overline{s \tau \tau}+\bar{\beta}\left(\bar{b}_{1} \bar{v}_{, \bar{\tau}}+\bar{b}_{2} \bar{v}_{, \overline{s \tau}}-\bar{v}_{,}, \bar{s} \overline{s \tau}\right) \\
& =\bar{t}_{0}\left(\bar{b}_{3} \bar{v}+\bar{b}_{4} \bar{v}_{, \bar{s}}+\bar{b}_{5} \bar{v}_{, \bar{s} \bar{s}}+\bar{b}_{6} \bar{v}_{, \bar{s} s \bar{s}}-\bar{v}_{, \bar{s} \bar{s} \bar{s}}\right)+\bar{b}_{7} \bar{p}_{1}+\bar{b}_{8} \bar{p}_{1, \bar{s}}
\end{aligned}
$$

By the assumption of harmonic motion for $\bar{v}$ and $\bar{p}_{1}$, and the application of separation of variables,

$$
\begin{aligned}
& \bar{v}(\bar{s}, \bar{\tau})=V(\bar{s}) e^{\lambda \bar{\tau}} \\
& \bar{p}_{1}(\bar{s}, \bar{\tau})=P(\bar{s}) e^{\lambda \bar{\tau}}
\end{aligned}
$$

Equation (4.18) becomes

$\left(\lambda^{2}+\bar{\beta} \lambda\right)\left(\bar{b}_{1} V+\bar{b}_{2} V^{\prime \prime}-V^{\prime \prime}\right)=\bar{t}_{0}\left(\bar{b}_{3} V+\bar{b}_{4} V^{\prime}+\bar{b}_{5} V^{\prime \prime}+\bar{b}_{6} V^{\prime \prime \prime}-V^{\prime \prime \prime \prime}\right)+\bar{b}_{7} P+\bar{b}_{8} P^{\prime}$

where the primes mean the ordinary derivatives with respect to $\bar{s}$.

Since there is no tangential or outward normal displacement at both fixed ends (anchored points), with the use of $w=\theta_{0, s}^{-1} v_{s}$ (see Equation (4.10)), the boundary conditions for Equation (4.21) are

$$
V(0)=0 ; \quad V^{\prime}(0)=0 ; \quad V\left(\bar{s}_{0}\right)=0 ; \quad V^{\prime}\left(\bar{s}_{0}\right)=0
$$

where $\bar{s}_{0}$ is the nondimensionalized curved perimeter of the membrane, which can be calculated from the given curved perimeter, $s_{0}$, and the base width, $l_{0}$, according to Equation (4.17).

The discretization of Equation (4.21) and the corresponding boundary conditions, Equation (4.22), is performed by dividing the curved perimeter into $m+1$ equal-length elements (Figure 4.2) with 
Equation (4.21) satisfied approximately at all the $m$ internal free nodes using a finite difference scheme. The discretized equations of motion and the boundary conditions are

$$
\begin{aligned}
& \left(\lambda^{2}+\bar{\beta} \lambda\right)\left(\bar{b}_{1 i} V_{i}+\bar{b}_{2 i} V_{i}^{\prime}-V_{i}^{\prime \prime}\right) \\
& =\bar{t}_{0}\left(\bar{b}_{3 i} V_{i}+\bar{b}_{4 i} V_{i}^{\prime}+\bar{b}_{5 i} V_{i}^{\prime \prime}+\bar{b}_{6 i} V_{i}^{\prime \prime \prime}-V_{i}^{\prime \prime \prime \prime}\right)+\bar{b}_{7 i} P_{i}+\bar{b}_{8 i} P_{i}^{\prime}
\end{aligned} \quad(i=1,2, \ldots, m)
$$

$V_{0}=0 ; \quad V_{0}^{\prime}=0 ; \quad V_{m+1}=0 ; \quad V_{m+1}^{\prime}=0$

Let $\bar{h}$ be the uniform curved spacing length between neighboring nodes on the dam. A finite difference scheme which is based on a central difference of order $O\left(\bar{h}^{2}\right)$, with exceptions where forward and backward differences are needed at Node 1 and Node $\mathrm{m}$ (shown in Appendix A), is applied to those terms with derivatives of $V_{i}$ and $P_{i}$, the coefficients $\left(\bar{b}_{1}, \bar{b}_{2 i}, \ldots, \bar{b}_{8 i}\right)$ in the equations of motion, Equation (4.23), and the boundary conditions, Equation (4.24). From Equation (4.24),

$V_{0}=0 ; \quad V_{-1}=V_{1} ; \quad V_{m+1}=0 ; \quad V_{m+2}=V_{m}$

The terms $V_{-1}$ and $V_{m+2}$ do not physically exist but they help establish the following equations of motion. From Equations (4.23), (4.25) and the finite difference scheme in Appendix A,

$$
\left(\lambda^{2}+\bar{\beta} \lambda\right) \sum_{j=1}^{m} B_{i j} V_{j}=\sum_{j=1}^{m} C_{i j} V_{j}+\sum_{k=0}^{m+1} D_{i ; k+1} P_{k} \quad \quad(i=1,2, \ldots, m)
$$

where the index $k$ is different from the index $j$ since the additional external pressure caused by the membrane vibration contributes at Nodes 0 and $m+1$ (fixed points), too. Semicolons (;) are used between the subscripts to avoid confusion wherever arithmetic operations appear as part of the subscripts, e.g., $i-1, i+1$, for the coefficients in Equation (4.26), $B_{i j}, C_{i j}$ and $D_{i ; k+1}$, as shown below: 


$$
\begin{aligned}
& B_{i ; i-1}=-\frac{\bar{b}_{2 i}}{2 \bar{h}}-\frac{1}{\bar{h}^{2}} \\
& (i=2,3, \ldots, m) \\
& B_{i i}=\bar{b}_{1 i}+\frac{2}{\bar{h}^{2}} \\
& (i=1,2, \ldots, m) \\
& B_{i ; i+1}=\frac{\bar{b}_{2 i}}{2 \bar{h}}-\frac{1}{\bar{h}^{2}} \\
& (i=1,2, \ldots, m-1) \\
& \text { Other } B_{i j}=0 \\
& C_{i ; i-2}=\bar{t}_{0}\left(\frac{-\bar{b}_{6 i}}{2 \bar{h}^{3}}-\frac{1}{\bar{h}^{4}}\right) \\
& (i=3,4, \ldots, m) \\
& C_{i ; i-1}=\bar{t}_{0}\left(\frac{-\bar{b}_{4 i}}{2 \bar{h}}+\frac{\bar{b}_{5 i}}{\bar{h}^{2}}+\frac{\bar{b}_{6 i}}{\bar{h}^{3}}+\frac{4}{\bar{h}^{4}}\right) \\
& (i=2,3, \ldots, m) \\
& C_{i i}=\bar{t}_{0}\left(\bar{b}_{3 i}-\frac{2 \bar{b}_{5 i}}{\bar{h}^{2}}-\frac{6}{\bar{h}^{4}}\right) \\
& (i=2,3, \ldots, m-1) \\
& C_{i ; i+1}=\bar{t}_{0}\left(\frac{\bar{b}_{4 i}}{2 \bar{h}}+\frac{\bar{b}_{5 i}}{\bar{h}^{2}}-\frac{\bar{b}_{6 i}}{\bar{h}^{3}}+\frac{4}{\bar{h}^{4}}\right) \\
& (i=1,2, \ldots, m-1) \\
& C_{i ; i+2}=\bar{t}_{0}\left(\frac{\bar{b}_{6 i}}{2 \bar{h}^{3}}-\frac{1}{\bar{h}^{4}}\right) \\
& (i=1,2, \ldots, m-2) \\
& C_{11}=\bar{t}_{0}\left(\bar{b}_{31}-\frac{2 \bar{b}_{51}}{\bar{h}^{2}}-\frac{\bar{b}_{61}}{2 \bar{h}^{3}}-\frac{7}{\bar{h}^{4}}\right) ; \quad C_{m m}=\bar{t}_{0}\left(\bar{b}_{3 m}-\frac{2 \bar{b}_{5 m}}{\bar{h}^{2}}+\frac{\bar{b}_{6 m}}{2 \bar{h}^{3}}-\frac{7}{\bar{h}^{4}}\right)
\end{aligned}
$$

Other $C_{i j}=0$

$D_{i i}=-\frac{\bar{b}_{8 i}}{2 \bar{h}} ; \quad D_{i ; i+1}=\bar{b}_{7 i} ; \quad D_{i ; i+2}=\frac{\bar{b}_{8 i}}{2 \bar{h}}$

Other $D_{i j}=0$ 
where

$$
\begin{aligned}
& \bar{b}_{1 j}=\bar{a}_{1 j}^{2} ; \quad \bar{b}_{2 j}=2 \frac{\bar{a}_{2 j}}{\bar{a}_{1 j}} ; \quad \bar{b}_{3 j}=\bar{a}_{2 j}^{2}-\bar{a}_{1 j} \bar{a}_{3 j} ; \\
& \bar{b}_{4 j}=\bar{a}_{2 j} \bar{a}_{8 j}-\bar{a}_{1 j} \bar{a}_{5 j}=8 \frac{\bar{a}_{2 j}^{3}}{\bar{a}_{1 j}^{3}}-7 \frac{\bar{a}_{2 j} \bar{a}_{3 j}}{\bar{a}_{1 j}^{2}}+\frac{\bar{a}_{4 j}}{\bar{a}_{1 j}}-\bar{a}_{1 j} \bar{a}_{2 j} ; \\
& \bar{b}_{5 j}=\bar{a}_{2 j} \bar{a}_{9 j}-\bar{a}_{1 j} \bar{a}_{6 j}=-8 \frac{\bar{a}_{2 j}^{2}}{\bar{a}_{1 j}^{2}}+3 \frac{\bar{a}_{3 j}}{\bar{a}_{1 j}}-\bar{a}_{1 j}^{2} ; \\
& \bar{b}_{6 j}=\frac{\bar{a}_{2 j}}{\bar{a}_{1 j}}-\bar{a}_{1 j} \bar{a}_{7 j}=4 \frac{\bar{a}_{2 j}}{\bar{a}_{1 j}} ; \quad \bar{b}_{7 j}=-\bar{a}_{2 j} ; \quad \bar{b}_{8 j}=\bar{a}_{1 j}
\end{aligned}
$$

$$
(j=1,2, \ldots, m)
$$

according to Equations (4.16) and (4.17). The equations for $\bar{a}_{k j}(k=1,2,3,4 ; j=1,2, \ldots, m)$ are shown in Equations (A.4)-(A.7) in Appendix A.

The pressure term (the last term on the right hand side) in the discretized equations of motion, Equation (4.26), needs to be further related to the displacement terms (the other two terms in the same equation) in order to finish setting up an eigenvalue problem. This is accomplished by taking account of the Laplace's equation and the Bernoulli equation for the overflow domain shown in the next section.

\subsection{FLOW DOMAIN ANALYSIS}

As the inflatable dam performs small vibrations about the equilibrium state, the velocity potential of the fluid in the domain of analysis changes with time accordingly. By the assumption of incompressible, inviscid, and irrotational flow, the Laplace's equation applies to the overflow problem in the dynamic analysis, as it does in the static analysis in Chapter 3. By superposition, 
the total velocity potential can be expressed as the sum of the velocity potential obtained in the static analysis, $\phi_{0}$, and the additional one caused by the membrane vibration, $\phi_{1}$ :

$$
\phi=\phi_{0}+\phi_{1}
$$

The Bernoulli equation for the potential flow in the dynamic analysis which relates the additional pressure, $p_{1}$, and the additional velocity potential, $\phi_{1}$, is

$$
\frac{1}{\gamma}\left[p+\rho\left(\phi,,_{\tau}+\frac{\phi_{x}^{2}+\phi_{y}^{2}}{2}\right)\right]+y=e_{0}
$$

where $\rho$ is the density of the fluid, which equals the specific weight divided by the acceleration of gravity, $\frac{\gamma}{g} ; x$ and $y$ are the $\mathrm{x}$ and $\mathrm{y}$ coordinates of the fluid particle, respectively; $p$ is the total pressure in the flow; $\phi_{,}$is the time derivative of the total velocity potential of the flow; $\phi, x$ and $\phi_{, y}$ are the total flow velocities in the $x$ and $y$ directions, respectively; and $e_{0}$ is the specified total head, which is a constant. In this Bernoulli equation, the right hand side which has to be space independent (e.g., Sabersky et al., 1989) takes the value of the given total head. Let $y_{0}$ and $y_{1}$ be the y-coordinates of the fluid particle in consideration from the static and dynamic analysis, respectively, and we can rewrite Equation (4.32) with the help of Equation (4.31). This gives

$$
\frac{1}{\gamma}\left\{p_{0}+p_{1}+\rho\left[\phi_{0, \tau}+\phi_{1, \tau}+\frac{\left(\phi_{0, x}+\phi_{1, x}\right)^{2}}{2}+\frac{\left(\phi_{0, y}+\phi_{1, y}\right)^{2}}{2}\right]\right\}+y_{0}+y_{1}=e_{0}
$$

where the variables with the subscript 0 refer to those in the static analysis while the variables with the subscript 1 refer to the additional variations caused by the membrane vibration. On the other hand, the Bernoulli equation in the static analysis is

$$
\frac{1}{\gamma}\left[p_{0}+\rho\left(\frac{\phi_{0, x}^{2}}{2}+\frac{\phi_{0, y}^{2}}{2}\right)\right]+y_{0}=e_{0}
$$

Subtracting Equation (4.34) from Equation (4.33), recalling that $\phi_{0, \tau}$ is zero, and neglecting the highest order terms in $\phi_{1}$, we find that the Bernoulli equation becomes 
$\frac{1}{\gamma}\left[p_{1}+\rho\left(\phi_{1, \tau}+\phi_{0, x} \phi_{1, x}+\phi_{0, y} \phi_{1, y}\right)\right]+y_{1}=0$

Since the Laplace's equation holds for the additional velocity potential as well as the total velocity potential (Equation (4.31)), the following Laplace's equation and boundary conditions (see Figure 4.3) for $\phi_{1}$ are used in the dynamic analysis:

$$
\begin{aligned}
& \nabla^{2} \phi_{1}=0 \quad \text { in Flow Domain } \\
& \phi_{1}=0 \quad \text { on Free Surface } \\
& \phi_{1}=0 \quad \text { on Inflow Boundary } \\
& \phi_{1}=0 \quad \text { on Outflow Boundary } \\
& \phi_{1, n}=0 \quad \text { on Bed Bottoms } \\
& \phi_{1, n}=-\left(w_{,_{\tau}}+\phi_{0, s} w,_{, 5}\right) \\
& =-\left[a_{1}^{-1} v_{, s \tau}+\phi_{0, s}\left(-a_{1}^{-2} a_{2} v_{, s}+a_{1}^{-1} v_{, s s}\right)\right] \quad \text { on Dam - Flow Interface }
\end{aligned}
$$

where $n$ is the unit outward normal to the surface enclosing the flow domain and $\phi_{0, s}$ is the flow velocity along the curved coordinate, $s$, in the static analysis. Compared with the Bernoulli equation (Equation (4.35)), Equation (4.37) indicates no y-coordinate change (wave motion) on the free surface with fixed atmospheric pressure; Equations (4.38) and (4.39) show that the effect on $\phi_{1}$ due to the membrane vibration diminishes at the far upstream inflow and far downstream outflow boundaries; Equation (4.40) verifies that the fixed bed is part of the streamlines; and Equation (4.41) shows the compatibility condition (kinetic boundary condition) between the movements of the membrane and the fluid particles in the normal direction (Bisplinghoff and Ashley, 1962). The relation between $v$ and $w$ in Equation (4.10) is used again to make the second equal sign in Equation (4.41). 
In addition to Equation (4.17), the following variables are introduced in order to nondimensionalize the Bernoulli equation (Equation (4.35)), the Laplace's equation (Equation (4.36)), and the corresponding boundary conditions (Equations (4.37)-(4.41)) for the overflow problem:

$\bar{\rho}=\frac{l_{0}}{\mu} \rho ; \quad \bar{n}=\frac{n}{l_{0}} ; \quad \bar{x}=\frac{x}{l_{0}} ; \quad \bar{y}=\frac{y}{l_{0}} ; \quad \bar{y}_{1}=\frac{y_{1}}{l_{0}} ;$

$\bar{\phi}_{0}=\sqrt{\frac{\mu}{\gamma}} \frac{1}{l_{0}^{2}} \phi_{0} ; \quad \bar{\phi}_{0, \bar{s}}=\sqrt{\frac{\mu}{\gamma}} \frac{1}{l_{0}} \phi_{0, s} ;$

$\bar{\phi}_{0, \bar{x}}=\sqrt{\frac{\mu}{\gamma}} \frac{1}{l_{0}} \phi_{0, x} ; \quad \bar{\phi}_{0, \bar{y}}=\sqrt{\frac{\mu}{\gamma}} \frac{1}{l_{0}} \phi_{0, y} ;$

$\bar{\phi}_{1}=\sqrt{\frac{\mu}{\gamma}} \frac{1}{l_{0}^{2}} \phi_{1} ; \quad \bar{\phi}_{1, \bar{n}}=\sqrt{\frac{\mu}{\gamma}} \frac{1}{l_{0}} \phi_{1, n} ; \quad \bar{\phi}_{1, \bar{s}}=\sqrt{\frac{\mu}{\gamma}} \frac{1}{l_{0}} \phi_{1, s} ;$

$\bar{\phi}_{1, \bar{x}}=\sqrt{\frac{\mu}{\gamma}} \frac{1}{l_{0}} \phi_{1, x} ; \quad \bar{\phi}_{1, \bar{y}}=\sqrt{\frac{\mu}{\gamma}} \frac{1}{l_{0}} \phi_{1, y} ; \quad \bar{\phi}_{1, \bar{\tau}}=\frac{\mu}{\gamma l_{0}^{2}} \phi_{1, \tau}$

Thus,

$\bar{p}_{1}+\bar{\rho}\left(\bar{\phi}_{1, \bar{\tau}}+\bar{\phi}_{0, \bar{x}} \bar{\phi}_{1, \bar{x}}+\bar{\phi}_{0, \bar{y}} \bar{\phi}_{1, \bar{y}}\right)+\bar{y}_{1}=0$

$\nabla^{2} \bar{\phi}_{1}=0 \quad$ in Flow Domain

$\bar{\phi}_{1}=0 \quad$ on Free Surface, Inflow Boundary, Outflow Boundary

$\bar{\phi}_{1, \bar{n}}=0 \quad$ on Bed Bottoms

$\bar{\phi}_{1, \bar{n}}=-\left[\frac{\bar{v}_{\overline{s \tau}_{\bar{s}}}}{\bar{a}_{1}}+\bar{\phi}_{0, \bar{s}}\left(-\frac{\bar{a}_{2}}{\bar{a}_{1}^{2}} \bar{v}, \bar{s}+\frac{\bar{v}_{, \bar{s} s}}{\bar{a}_{1}}\right)\right] \quad$ on Dam - Flow Interface

The additional potential caused by the membrane vibration, $\bar{\phi}_{1}$, is assumed to be in the same exponential form as that for $v$ (see Equation (4.19)) when separation of variables applies: 
$\bar{\phi}_{1}(\bar{x}, \bar{y}, \bar{\tau})=\Phi(\bar{x}, \bar{y}) e^{\lambda \bar{\tau}}$

By Equations (4.19) and (4.48), the potential problem (Equations (4.44)-(4.49)) becomes

$\nabla^{2} \Phi=0 \quad$ in Flow Domain

$\Phi=0 \quad$ on Free Surface, Inflow Boundary, Outflow Boundary

$\Phi, \bar{n}=0 \quad$ on Bed Bottoms

$\Phi, \bar{n}=-\left[\lambda \frac{V^{\prime}}{\bar{a}_{1}}+\bar{\phi}_{0, \bar{s}}\left(-\frac{\bar{a}_{2}}{\bar{a}_{1}^{2}} V^{\prime}+\frac{V^{\prime \prime}}{\bar{a}_{1}}\right)\right] \quad$ on Dam-Flow Interface

After discretization, the boundary conditions (Equations (4.50)-(4.52)) turn into

$\Phi_{F}=0 \quad$ on Free Surface ; $\quad \Phi_{I}=0$ on Inflow Boundary ;

$\Phi_{O}=0 \quad$ on Outflow Boundary

$\Phi_{B U, \bar{n}}=0 \quad$ on Upstream Bed Bottom; $\quad \Phi_{B D, \bar{n}}=0 \quad$ on Downstream Bed Bottom

$\Phi_{j, \bar{n}}=-\left[\lambda \frac{V_{j}^{\prime}}{\bar{a}_{1 j}}+\bar{\phi}_{0 j,}\left(-\frac{\bar{a}_{2 j}}{\bar{a}_{1 j}^{2}} V_{j}^{\prime}+\frac{V_{j}^{\prime \prime}}{\bar{a}_{1 j}}\right)\right]$

$=\sum_{k=1}^{m}\left(M_{j+1 ; k}+\lambda N_{j+1 ; k}\right) V_{k} \quad$ on Dam-Flow Interface

$(j=0,1, \ldots, m+1)$

where $\Phi, \Phi_{F}, \Phi_{I}, \Phi_{O}, \Phi_{B U}$, and $\Phi_{B D}$ are the $\Phi$ values designated at the nodes of the section of the membrane, the free surface, the inflow boundary, the outflow boundary, and the two bed bottoms (upstream and downstream), respectively. $\Phi_{j}$ represents the value of $\Phi$ at Node $j$ of the membrane section. The equations for $M$ and $N$ are shown in Appendix A. 
A boundary value problem is established by Equations (4.49) and (4.53)-(4.55) and solved by a boundary element method using linear elements (Liggett and Liu, 1983). By Equation (2.34) on Page 25 in Liggett and Liu (1983), the system equation from the boundary element method is

$$
\begin{aligned}
& {[R]\left[\begin{array}{lllllll}
\Phi & \Phi_{B U} & \Phi_{I} & \Phi_{F} & \Phi_{O} & \Phi_{B D}
\end{array}\right]^{T}} \\
& =[L]\left[\begin{array}{lllllll}
\Phi, \bar{n} & \Phi_{B U, \bar{n}} & \Phi_{I, \bar{n}} & \Phi_{F, \bar{n}} & \Phi_{O, \bar{n}} & \Phi_{B D, \bar{n}}
\end{array}\right]^{T}
\end{aligned}
$$

where $R$ and $L$ are the coefficient matrices for $\Phi$ and $\Phi, \bar{n}$, which are calculated by the node coordinates in the static solution and the boundary element method, with the first node set at the most downstream node on the dam and with the remaining node numbers counted clockwise. Moving the unknowns to the left hand side and exchanging the corresponding rows between $R$ and $L$ with appropriate sign changes, we have the new matrices, $\bar{R}$ and $\bar{L}$, and Equation (4.56) becomes

$$
\begin{aligned}
& {[\bar{R}]\left[\begin{array}{lllllll}
\Phi & \Phi_{B U} & \Phi_{l, \bar{n}} & \Phi_{F, \bar{n}} & \Phi_{O, \bar{n}} & \Phi_{B D}
\end{array}\right]^{T}} \\
& =[\bar{L}]\left[\begin{array}{llllll}
\Phi, \bar{n} & \Phi_{B U, \bar{n}} & \Phi_{I} & \Phi_{F} & \Phi_{O} & \Phi_{B D, \bar{n}}
\end{array}\right]^{T}
\end{aligned}
$$

Since this is a mixed boundary value problem (Neumann and Dirichlet), the inverse of $\bar{R}$ in Equation (4.57) exists and we have

$\left[\begin{array}{llllll}\Phi & \Phi_{B U} & \Phi_{I, \bar{n}} & \Phi_{F, \bar{n}} & \Phi_{O, \bar{n}} & \Phi_{B D}\end{array}\right]^{T}$

$=[\bar{R}]^{-1}[\bar{L}]\left[\Phi, \bar{n} \quad \Phi_{B U, \bar{n}} \quad \Phi_{l} \quad \Phi_{F} \quad \Phi_{O} \quad \Phi_{B D, \bar{n}}\right]^{T}$

$=[Q]\left[\begin{array}{llllll}\Phi, \bar{n} & \Phi_{B U, \bar{n}} & \Phi_{I} & \Phi_{F} & \Phi_{O} & \Phi_{B D, \bar{n}}\end{array}\right]^{T}$

where $[Q]=[\bar{R}]^{-1}[\bar{L}]$. From Equations (4.53) and (4.54), the elements of the last matrix on the right hand side in Equation (4.58) are zero except for $\Phi, \bar{n}$ (see Figure 4.3). Therefore,

$\Phi_{i}=\sum_{j=0}^{m+1} Q_{i+1 ; j+1} \Phi_{j, \bar{n}}$

$(i=0,1, \ldots, m+1)$ 
Only the first $m+2$ rows and the first $m+2$ columns in $Q$ which correspond to the nodes on the membrane are used in the formulation of Equation (4.59). Combining Equations (4.55) and (4.59), we have

$$
\begin{aligned}
& \Phi_{i}=\sum_{j=0}^{m+1} \sum_{k=1}^{m}\left(Q_{i+1 ; j+1} M_{j+1 ; k}+\lambda Q_{i+1 ; j+1} N_{j+1 ; k}\right) V_{k} \\
& =\sum_{k=1}^{m}\left(\bar{M}_{i+1 ; k}+\lambda \bar{N}_{i+1 ; k}\right) V_{k}
\end{aligned}
$$$$
(i=0,1, \ldots, m+1)
$$

where $\bar{M}_{i+1 ; k}=\sum_{j=0}^{m+1} Q_{i+1 ; j+1} M_{j+1 ; k}$ and $\bar{N}_{i+1 ; k}=\sum_{j=0}^{m+1} Q_{i+1 ; j+1} N_{j+1 ; k}$.

Now that $\Phi$ and $V$ are related by Equation (4.59), we have to further relate $\Phi$ and $P$ in order to solve the equation of motion for the membrane (Equation (4.26)), with the help of the Bernoulli equation. The Bernoulli equation derived at the beginning of this section (Equation (4.35)) is

$\frac{1}{\gamma}\left[p_{1}+\rho\left(\phi_{1, \tau}+\phi_{0, x} \phi_{1, x}+\phi_{0, y} \phi_{1, y}\right)\right]+y_{1}=0$

When the Bernoulli equation is employed on the interface of the dam membrane and the flow, the term $y_{1}$, the additional dynamic displacement of the fluid in the $y$ direction, can be related to $v$ and $w$, the tangential and normal displacements of the membrane, as follows:

$y_{1}=v \sin \theta_{0}+w \cos \theta_{0}=v \sin \theta_{0}+a_{1}^{-1} v, s \cos \theta_{0}$

where $\theta_{0}$ is the slope of the dam shape, and $a_{1}^{-1}$ is defined in Equation (4.12), both of which are known from the result of the static analysis. For small vibrations, the velocity terms in Equation (4.35) are

$\phi_{0, x} \phi_{1, x}+\phi_{0, y} \phi_{1, y} \cong \phi_{0, n} \phi_{1, n}+\phi_{0, s} \phi_{1, s}=\phi_{0, s} \phi_{1, s}$ 
since $\phi_{0, n}=0$ at the interface in the static analysis. By Equations (4.61) and (4.62), Equation (4.35) becomes

$\frac{1}{\gamma}\left[p_{1}+\rho\left(\phi_{1, \tau}+\phi_{0, s} \phi_{1, s}\right)\right]+v \sin \theta_{0}+a_{1}^{-1} v, s \cos \theta_{0}=0$

Using the nondimensionalized variables listed in Equations (4.17) and (4.42), Equation (4.63) becomes

$\bar{p}_{1}+\bar{\rho}\left(\bar{\phi}_{1, \bar{\tau}}+\bar{\phi}_{0, \bar{s}} \bar{\phi}_{1, \bar{s}}\right)+\bar{v} \sin \bar{\theta}_{0}+\frac{\cos \bar{\theta}_{0}}{\bar{a}_{1}} \bar{v}_{\bar{s}}=0$

By separation of variables (Equations (4.19), (4.20) and (4.48)),

$P+\bar{\rho}\left(\lambda \Phi+\bar{\phi}_{0, \bar{s}} \Phi, \bar{s}\right)+V \sin \bar{\theta}_{0}+\frac{\cos \bar{\theta}_{0}}{\bar{a}_{1}} V^{\prime}=0$

By discretization, Equation (4.65) turns into

$P_{i}+\bar{\rho}\left(\lambda \Phi_{i}+\bar{\phi}_{0 i, \bar{s}} \Phi_{i, \bar{s}}\right)+V_{i} \sin \bar{\theta}_{0 i}+\frac{\cos \bar{\theta}_{0 l}}{\bar{a}_{1 i}} V_{i}^{\prime}=0$

$(i=0,1, \ldots, m+1)$

where $\Phi_{i, s}$ and other terms with ordinary or partial derivatives can be calculated by a finite difference method displayed in Appendix A. After the execution of this finite difference method, Equation (4.66) becomes

$P_{i}=-\left[\bar{\rho}\left(\lambda \Phi_{i}+\sum_{j=0}^{m+1} H_{i+1 ; j+1} \Phi_{j}\right)+\sum_{j=1}^{m} K_{i+1 ; j} V_{j}\right] \quad(i=0,1, \ldots, m+1)$

where $H$ and $K$ can be found in Appendix A. 


\subsection{EQUATION ASSEMBLING}

One equation of motion for the membrane (Equation (4.26)), one equation from Laplace's equation (Equation (4.60)), and one equation from the Bernoulli equation (Equation (4.67)) were derived in the last two sections and are ready to be assembled. Replacing the $P$ in Equation (4.26) with the one in Equation (4.67), we have

$$
\begin{aligned}
& \left(\lambda^{2}+\bar{\beta} \lambda\right) \sum_{j=1}^{m} B_{i j} V_{j}=\sum_{j=1}^{m}\left(C_{i j}-\sum_{k=0}^{m+1} D_{i ; k+1} K_{k+1 ; j}\right) V_{j} \\
& -\bar{\rho} \sum_{j=0}^{m+1}\left(\lambda D_{i ; j+1}-\sum_{k=0}^{m+1} D_{i ; k+1} H_{k+1 ; j+1}\right) \Phi_{j} \\
& =\sum_{j=1}^{m} \bar{C}_{i j} V_{j}-\bar{\rho} \sum_{j=0}^{m+1}\left(\lambda D_{i ; j+1}+\bar{H}_{i ; j+1}\right) \Phi_{j}
\end{aligned}
$$

where $\bar{C}_{i j}=C_{i j}-\sum_{k=0}^{m+1} D_{i ; k+1} K_{k+1 ; j}$ and $\bar{H}_{i ; j+1}=\sum_{k=0}^{m+1} D_{i ; k+1} H_{k+1 ; j+1}$. Then we substitute Equation (4.60) into Equation (4.68), and obtain

$$
\begin{aligned}
& \sum_{j=1}^{m}\left\{\lambda^{2}\left(B_{i j}+\bar{\rho} \sum_{k=0}^{m+1} D_{i ; k+1} \bar{N}_{k+1 ; j}\right)\right. \\
& +\lambda\left[\bar{\beta} B_{i j}+\bar{\rho} \sum_{k=0}^{m+1}\left(D_{i ; k+1} \bar{M}_{k+1 ; j}+\bar{H}_{i ; k+1} \bar{N}_{k+1 ; j}\right)\right] \\
& \left.+\left(\bar{\rho} \sum_{k=0}^{m+1} \bar{H}_{i ; k+1} \bar{M}_{k+1 ; j}-\bar{C}_{i j}\right)\right\} V_{j}=0
\end{aligned}
$$

which can be written in the form 
$\sum_{j=1}^{m}\left(\lambda^{2} E_{i j}+\lambda F_{i j}+G_{i j}\right) V_{j}=0$

$(i=1,2, \ldots, m)$

where $\quad E_{i j}=B_{i j}+\bar{\rho} \sum_{k=0}^{m+1} D_{i ; k+1} \bar{N}_{k+1 ; j} ; \quad F_{i j}=\bar{\beta} B_{i j}+\bar{\rho} \sum_{k=0}^{m+1}\left(D_{i ; k+1} \bar{M}_{k+1 ; j}+\bar{H}_{i ; k+1} \bar{N}_{k+1 ; j}\right) \quad ; \quad$ and $G_{i j}=\bar{\rho} \sum_{k=0}^{m+1} \bar{H}_{i ; k+1} \bar{M}_{k+1 ; j}-\bar{C}_{i j}$.

\subsection{EXAMPLES}

Introducing a variable $Z_{j}$,

$Z_{j}=\lambda V_{j}$

$$
(j=1,2, \ldots, m)
$$

we can rewrite Equation (4.70) as

$\sum_{j=1}^{m}\left(\lambda E_{i j} Z_{j}+F_{i j} Z_{j}+G_{i j} V_{j}\right)=0$

$(i=1,2, \ldots, m)$

The eigenvalue system established by Equations (4.71) and (4.72) doubles the size of the eigenvectors in the original problem (Equation (4.70)), and the order of $\lambda$ is reduced by one. Then a subroutine, named DGVCRG (IMSL, 1989), can be used to solve for all the eigenvalues and eigenvectors.

The examples shown here are based on the result of the static analysis of the example in Section 3.5. There are two variables which need to be assigned certain values, $\bar{\rho}$ (the fluid/dam density ratio 
parameter), and $\bar{\beta}$ (the structural damping parameter) in the coefficients of $V_{j}$ and $Z_{j}$ in Equation (4.72).

4.5.1 Example: $\bar{\rho}=1.0 ; \vec{\beta}=0.1$

According to Equation (4.19), the tangential displacement of the membrane is

$\bar{v}(\bar{s}, \bar{\tau})=V(\bar{s}) e^{\lambda \bar{\tau}}$

Let the solutions for $V$ and $\lambda$ be

$V=V_{r}+i V_{i}$

$\lambda=\lambda_{r}+i \lambda_{i}$

Both of these come from the result of eigenvalue analysis. We have

$\bar{v}=e^{\lambda_{r} \bar{\tau}}\left(V_{r} \cos \lambda_{i} \bar{\tau}-V_{i} \sin \lambda_{i} \bar{\tau}\right)$

after discarding the imaginary part of $\bar{v}$.

From the relation of $w$ and $v$ in Equation (4.10) and the use of the nondimensionalization and finite differences, we can obtain $\bar{w}$, which is the nondimensionalized outward normal displacement of the membrane. Combinations of the projections of $\bar{v}$ and $\bar{w}$ onto $\bar{x}_{0}$ and $\bar{y}_{0}$ give the displacements on these two axes for the membrane during vibration:

$\Delta \bar{x}_{0}=\bar{v} \cos \bar{\theta}_{0}-\bar{w} \sin \bar{\theta}_{0}$

$\Delta \bar{y}_{0}=\bar{v} \sin \bar{\theta}_{0}+\bar{w} \cos \bar{\theta}_{0}$

where $\bar{\theta}_{0}$ is the slope of the membrane. 
In the first example, $\bar{\rho}=1.0$ and $\bar{\beta}=0.1$. There are 19 free nodes on the membrane and thus 38 eigenvalues (Equations (4.71) and (4.72)). All 38 real parts of the eigenvalues $\left(\lambda_{r}\right)$ are found to be negative, so this system is stable. The eigenvalues come in conjugate pairs if the imaginary parts are not zero. We arrange the eigenvalues in the order of increasing absolute values of the imaginary parts, and assign the mode number accordingly (a conjugate pair is regarded as one mode only). The eigenvalues for the first four modes are

Mode 1: $-0.02492 \pm i(1.8250)$

Mode 2: $-0.02857 \pm i(3.4290)$

Mode 3: $-0.02217 \pm i(5.0640)$

Mode 4: $-0.02468 \pm i(6.5370)$

The period of vibration for each mode is computed by the following equation:

$T_{i}=\frac{2 \pi}{\lambda_{i}} \quad i=1,2, \ldots$

With the eigenvalues, eigenvectors (for $V$ ), and the method mentioned earlier in this section, we show the displacements of the membrane during vibration for Mode 1 in the first cycle in Figure 4.4. The dotted lines shown are for the equilibrium state. Figures 4.5 and 4.6 show the contributions of the real parts and the imaginary parts of the eigenvectors $\left(V_{r}\right.$ and $V_{i}$ ), respectively, to the displacements of the four modes. In other words, Figure 4.5 depicts the shapes at $\bar{\tau}=0$ and Figure 4.6 gives the shapes at $\bar{\tau}=\frac{\pi}{2 \lambda_{i}}$. These modes are not classical modes, and it is not necessarily true that the first mode has one node, the second mode has two nodes, and so on, at all times.

4.5.2 Example: $\bar{\rho}=12 ; \bar{\beta}=1$

This is another stable case with the eigenvalues for Modes 1-4 as follows:

Mode 1: $-0.0397 \pm i(0.9986)$

Mode 2: $-0.0956 \pm i(2.0930)$ 
Mode 3: $-0.0719 \pm i(3.2540)$

Mode 4: $-0.1396 \pm i(4.5300)$

Figures 4.7-4.11 show the displacements of the membrane in the first five cycles $\left(\bar{\tau}=0-\frac{19}{4} T_{1}\right)$ for Mode 1. A similar pattern is displayed in these figures. It is found that the shape of the dam moves toward the static solution (equilibrium shape, dotted line) as time goes on since the mode is asymptotically stable. Vibrations of Modes 2-4 during the first cycle are shown in Figures 4.12-4.14. More complicated shapes appear than those in Mode 1.

Figures 4.15-4.17 give time histories for the outward normal displacements at Nodes 27, 22, and 17 in Mode 1. These nodes are the three which divide the curved perimeter of the membrane into four sections of equal curved length, in the direction from upstream to downstream (see Figure 3.3). Since the real parts of the eigenvalues are negative, $\bar{w}$ vibrates with decreasing amplitude at all of the nodes.

4.5.3 Example: $\bar{\rho}=50 ; \bar{\beta}=1$

This is an example with instability:

Mode 1: -0.0927

Mode 2: 0.5399

Mode 3: $0.0109 \pm i(0.9023)$

Mode 4: $1.1710 \pm i(1.6670)$

Mode 5: $-0.2704 \pm i(1.8050)$

There is stability without oscillation in Mode 1, divergence in Mode 2, flutter in Modes 3 and 4, and stability with oscillation in Mode 5. The displacement diagrams are shown in Figures 4.18-4.22 for Modes 1-5, respectively. In Figures 4.20-4.22, the vibration is still for the first cycle, but in Figures 4.18 and 4.19 , some arbitrary time values are used since the periods for these two modes are infinity. 
The outward normal displacements at Nodes 27, 22 , and 17 in Mode 3, which is a flutter mode, are shown in Figures 23-25, respectively. The dam undergoes vibrations without limits. If we focus on the middle node (Node 22 ), $\bar{w}$ decays in Figure 4.26 (Mode 1, stability without oscillation), becomes divergent in Figure 4.27 (Mode 2, divergence), flutters in Figure 4.28 (Mode 4), and exhibits stability with oscillation in Figure 4.29 (Mode 5).

\subsubsection{Variation of $\lambda$ with $\bar{\beta}$}

In Example 4.5.1, $\bar{\beta}$ has to be greater than or equal to 0.06 for the system to be stable if $\bar{\rho}=1$; otherwise, positive real parts of the eigenvalues will appear. Similar lower bounds exist for Examples 4.5.2 and 4.5.3, which are 0.9 (if $\bar{\rho}=12$ ) and infinity (if $\bar{\rho}=50$ ). That is to say, there is no stable solution for $\bar{\rho}=50$ in this study.

In Sections 4.5.4 and 4.5.5, the modes are arranged somewhat differently. The order of increasing absolute values of the imaginary parts still applies, but all the eigenvalues are grouped pair by pair, including those with zero imaginary parts. The pair of eigenvalues with the smallest absolute values of the imaginary parts are assigned Mode A, the second smallest as Mode B, etc. The reason why this new arrangement is used is that if the imaginary parts become zero from nonzero values by changing either $\bar{\rho}$ or $\bar{\beta}$, the number of modes affected will be an even number.

The imaginary parts $\left(\lambda_{i}\right)$ and the real parts $\left(\lambda_{r}\right)$ of the eigenvalues change when $\bar{\beta}$ changes at $\bar{\rho}=1,5$, and 12 as in Figures 4.30-4.35 (the first four modes are shown). Generally speaking, increasing $\bar{\beta}$ causes decreasing $\lambda_{i}$ (Figures 4.30 and 4.32 , for example). The real parts become decreasing for increasing $\bar{\beta}$ as well; but for the lower modes (Modes $\mathrm{A}$ and $\mathrm{B}$ in Figures 4.31 and 4.33), $\lambda_{r}$ bifurcates when the imaginary parts reach zero. For Mode B in Figure 4.34, $\lambda_{i}$ hits zero but returns to the positive side immediately (at $\bar{\beta} \cong 9$ ). At the same $\bar{\beta}$ we find that $\lambda_{r}$ for Mode $\mathrm{B}$ intersects that for Mode A (Figure 4.35). Since this is where Mode B has zero imaginary parts, a bifurcation does exist for $\lambda_{r}$ (see the enlargement picture at the bottom half of Figure 4.35). Modes $\mathrm{A}$ and $\mathrm{B}$ merge right after the bifurcation for $\lambda_{r}$ of Mode $\mathrm{B}$. 


\subsubsection{Variation of $\lambda$ with $\bar{\rho}$}

In Example 4.5.1, $\bar{\rho}$ has to be less than or equal to 1 for the system to be stable if $\bar{\beta}=0.1$; otherwise, positive real parts of the eigenvalues will appear. Of course, $\bar{\rho}$ can not be zero, which is the case that the density of the water is zero or the density of the dam material is infinity. A similar upper bound for $\bar{\rho}$ in Examples 4.5.2 and 4.5.3 (both with $\bar{\beta}=1$ ) is 13. The highest $\bar{\rho}$ found to correspond to a stable vibration in this study is 26 at a very large $\bar{\beta}(1,000,000)$.

The variations of the imaginary parts and the real parts of the first four eigenvalues with $\bar{\rho}$ when $\bar{\beta}=0.1,1$, and 10 are shown in Figures $4.36-4.41$. When $\bar{\beta}$ is small $(0.1$ or 1$)$ increasing $\bar{\rho}$ brings decreasing $\lambda_{i}$ (Figures 4.36 and 4.38) and increasing $\lambda_{r}$ (Figures 4.37 and 4.39). All the first four modes carry positive real parts of the eigenvalues when $\bar{\rho}$ is greater than 2.3 in the case $\vec{\beta}=0.1$, which means instability.

Modes $\mathrm{A}$ and $\mathrm{B}$ in Figure 4.40 have zero $\lambda_{i}$ for quite a range of $\bar{\rho}$ but the Mode B curve rises at $\bar{\rho} \cong 10.5$ while the Mode A stays zero. Moreover, $\lambda_{r}$ at the same $\bar{\rho}$ in Figure 4.41 displays a merging behavior for Mode B, which is similar to that in Figure 4.35.

The bifurcation of the real part of an eigenvalue appears when the corresponding imaginary parts are driven from a positive number to zero. On the contrary, merging happens for the real parts when an imaginary part resumes its nonzero status. 


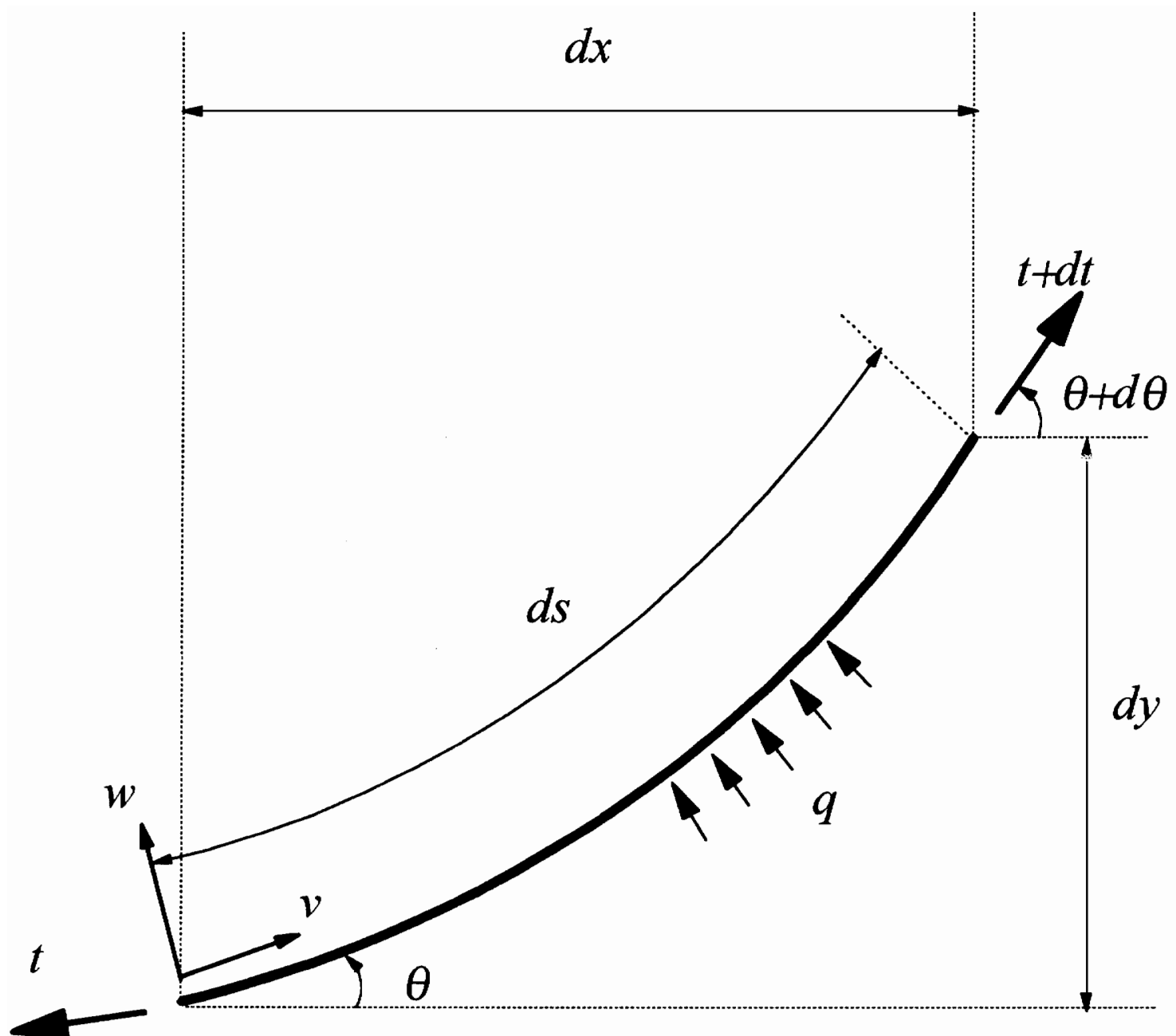

Figure 4.1 A Membrane Element in Vibration 


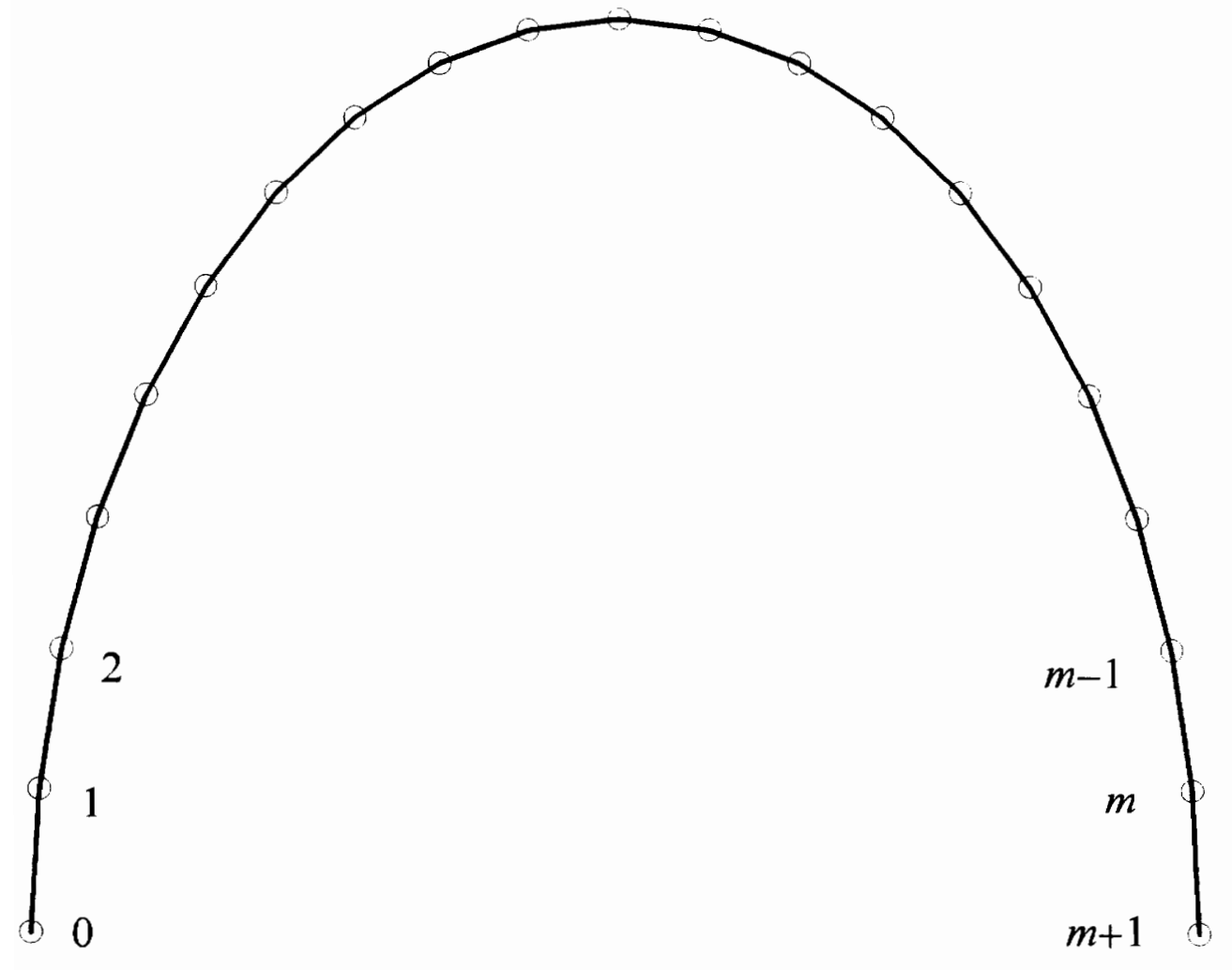

Figure 4.2 Membrane Node Setup 


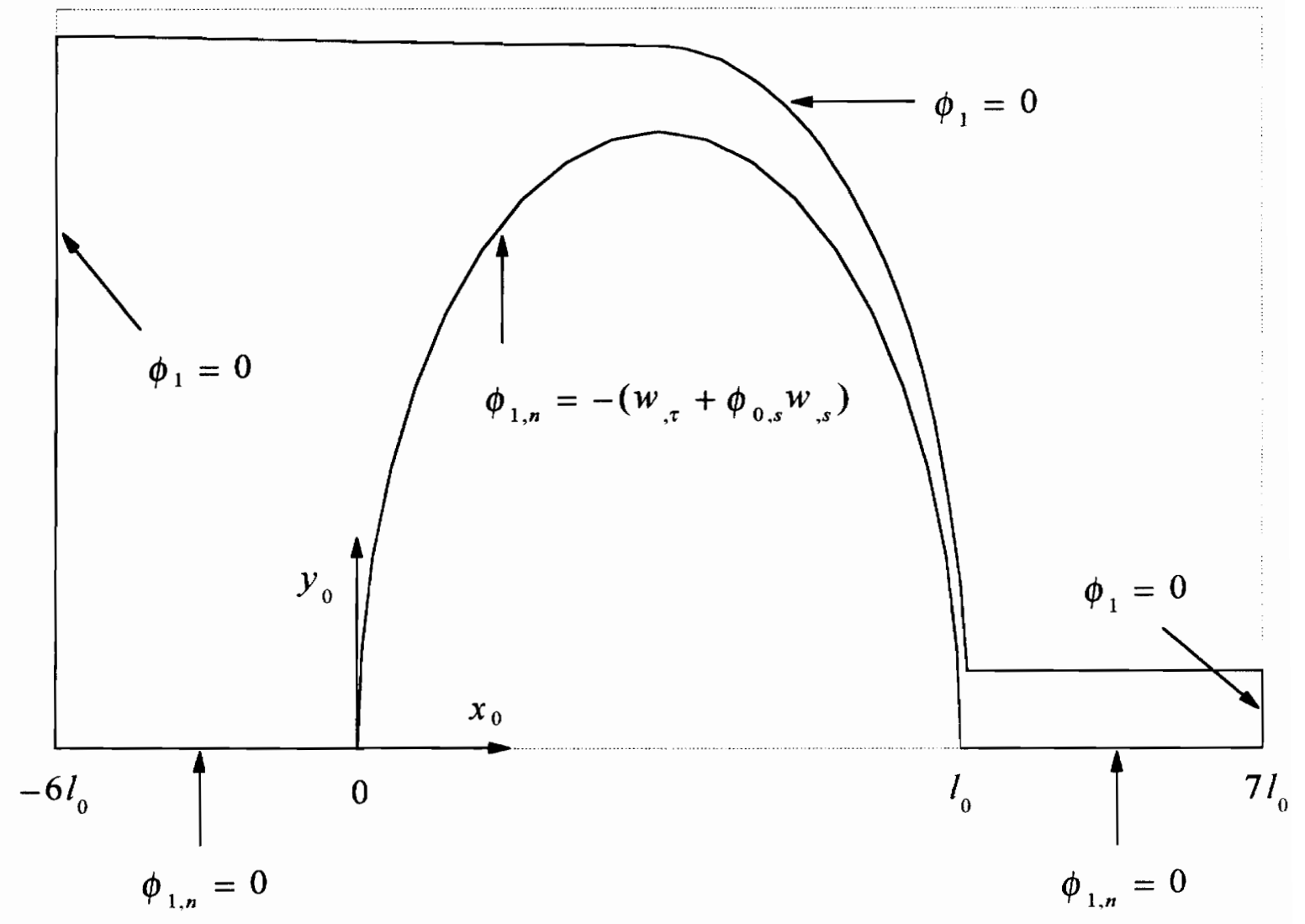

Figure 4.3 The Flow Domain in Dynamic Analysis 

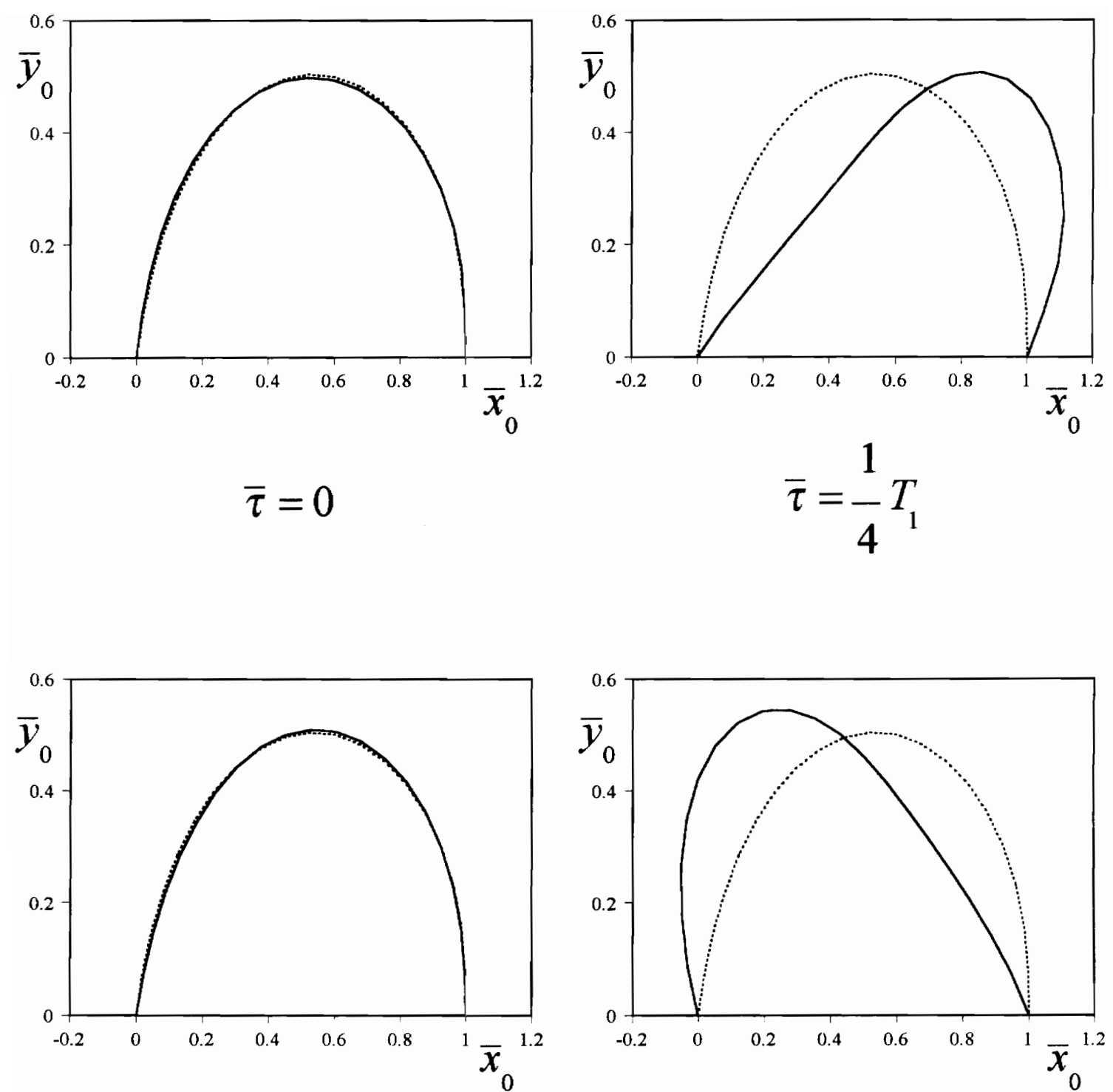

$$
\bar{\tau}=\frac{1}{2} T_{1}
$$

$$
\bar{\tau}=\frac{3}{4} T_{1}
$$

Figure 4.4 Example 4.5.1, Mode 1, Displacement with Time 


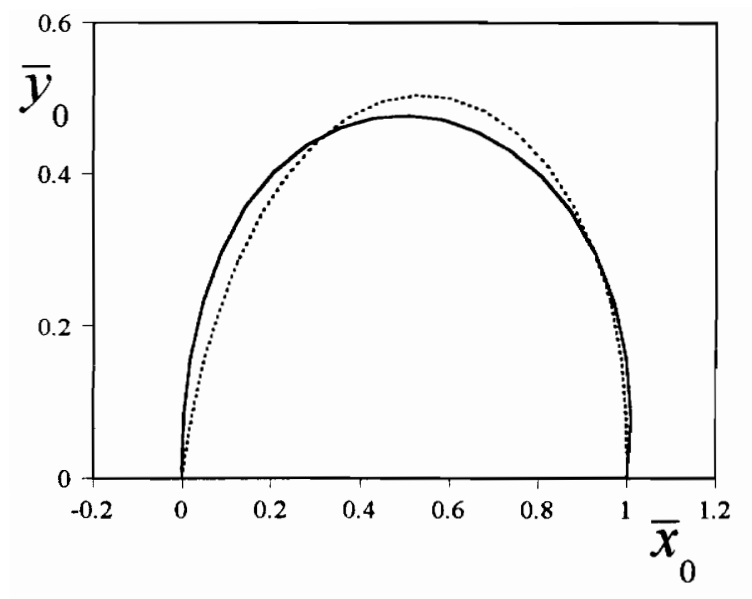

Mode 1

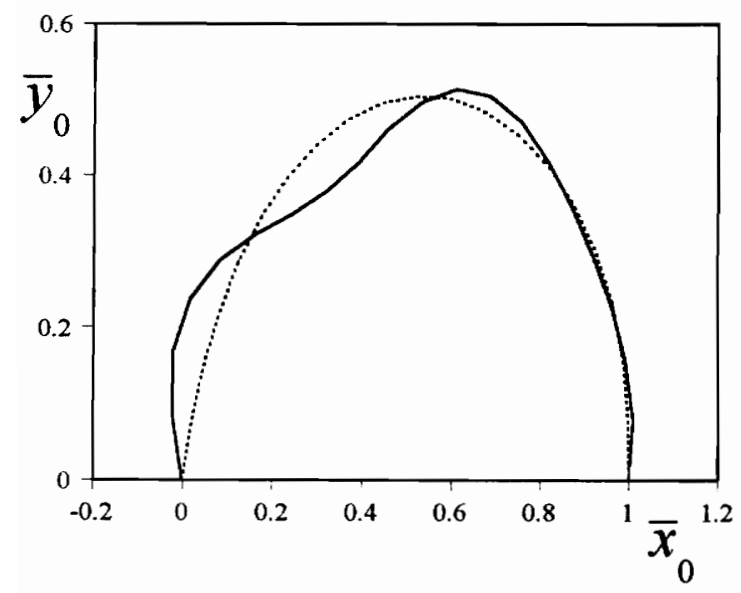

Mode 3

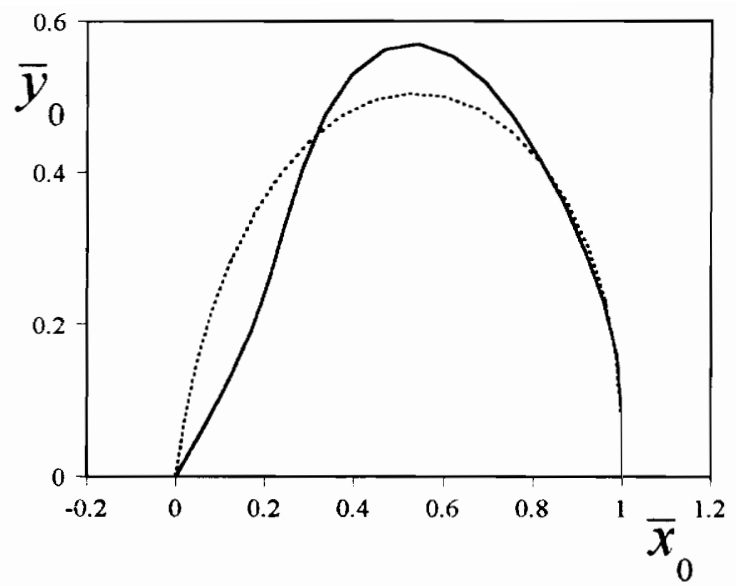

Mode 2

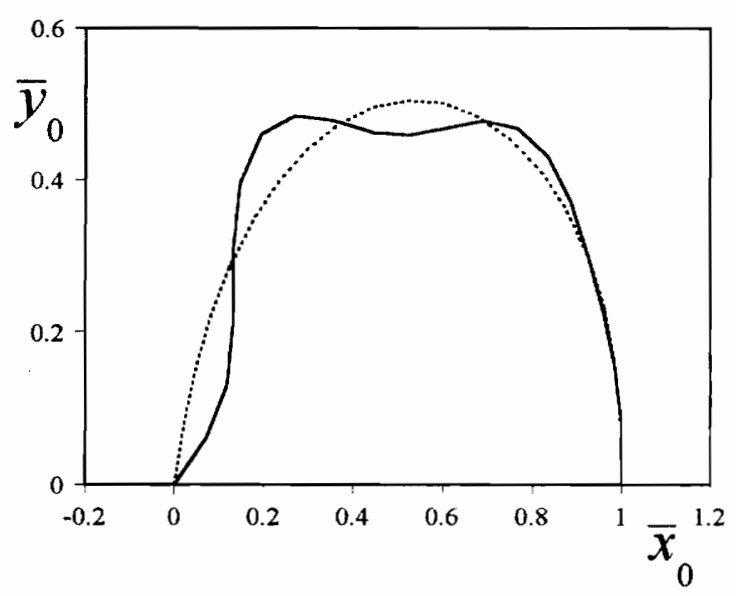

Mode 4

Figure 4.5 Example 4.5.1, Contribution of Real Parts of Eigenvectors 


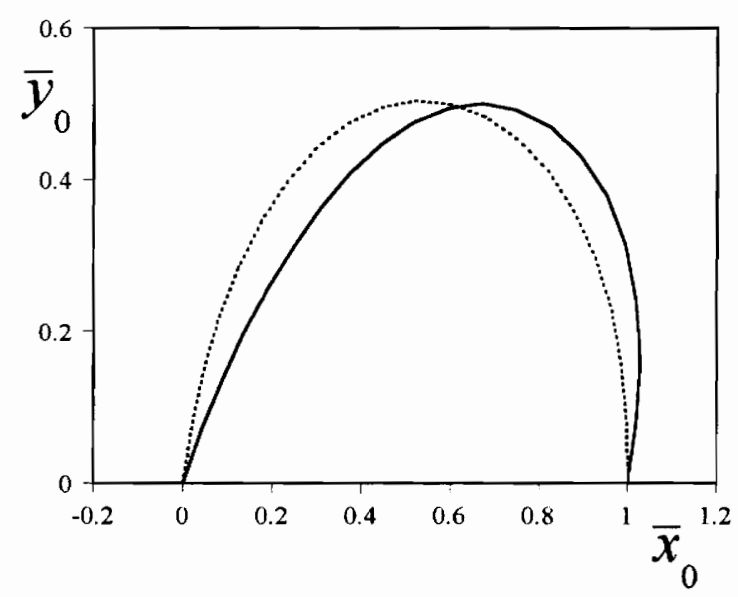

Mode 1

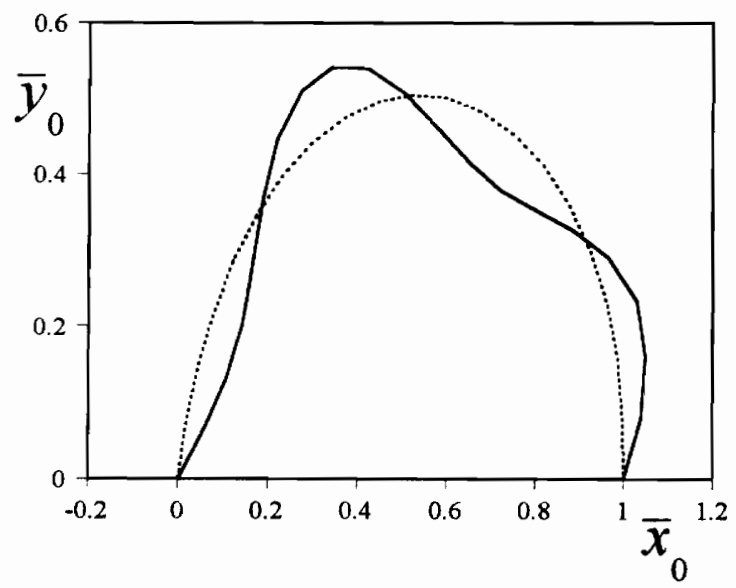

Mode 3

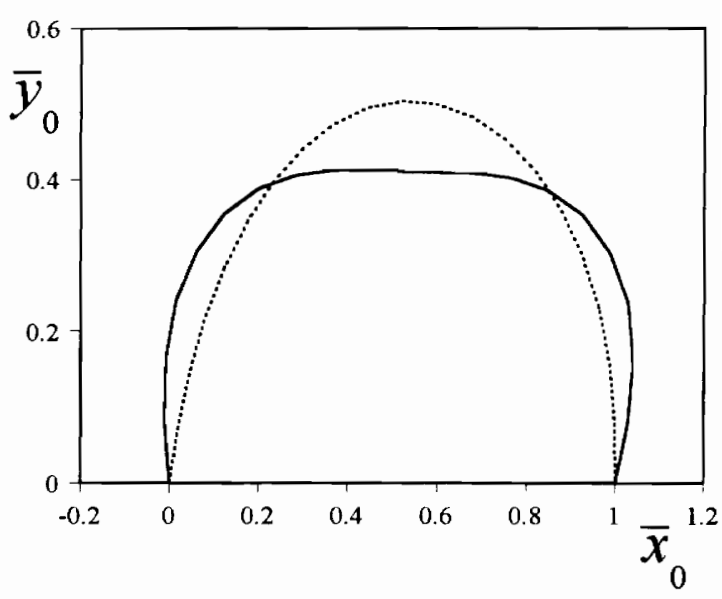

Mode 2

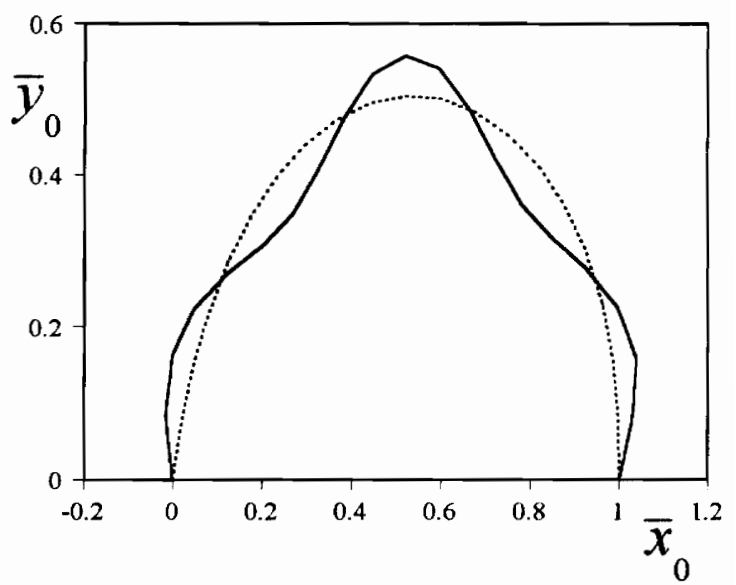

Mode 4

Figure 4.6 Example 4.5.1, Contribution of Imaginary Parts of Eigenvectors 

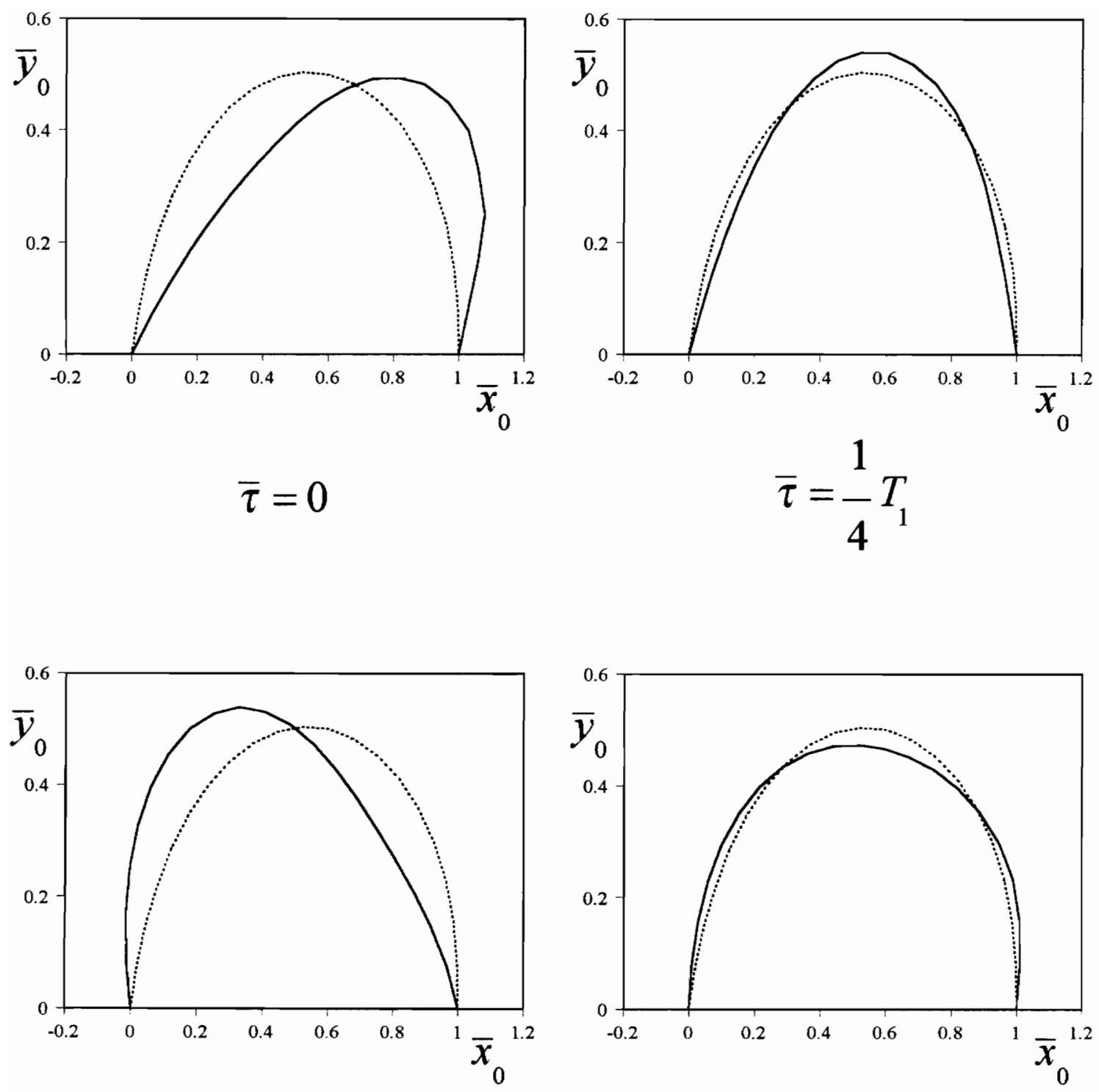

$$
\bar{\tau}=\frac{1}{2} T_{1}
$$

$$
\bar{\tau}=\frac{3}{4} T_{1}
$$

Figure 4.7 Example 4.5.2, Mode 1, Displacement with Time (First Cycle) 

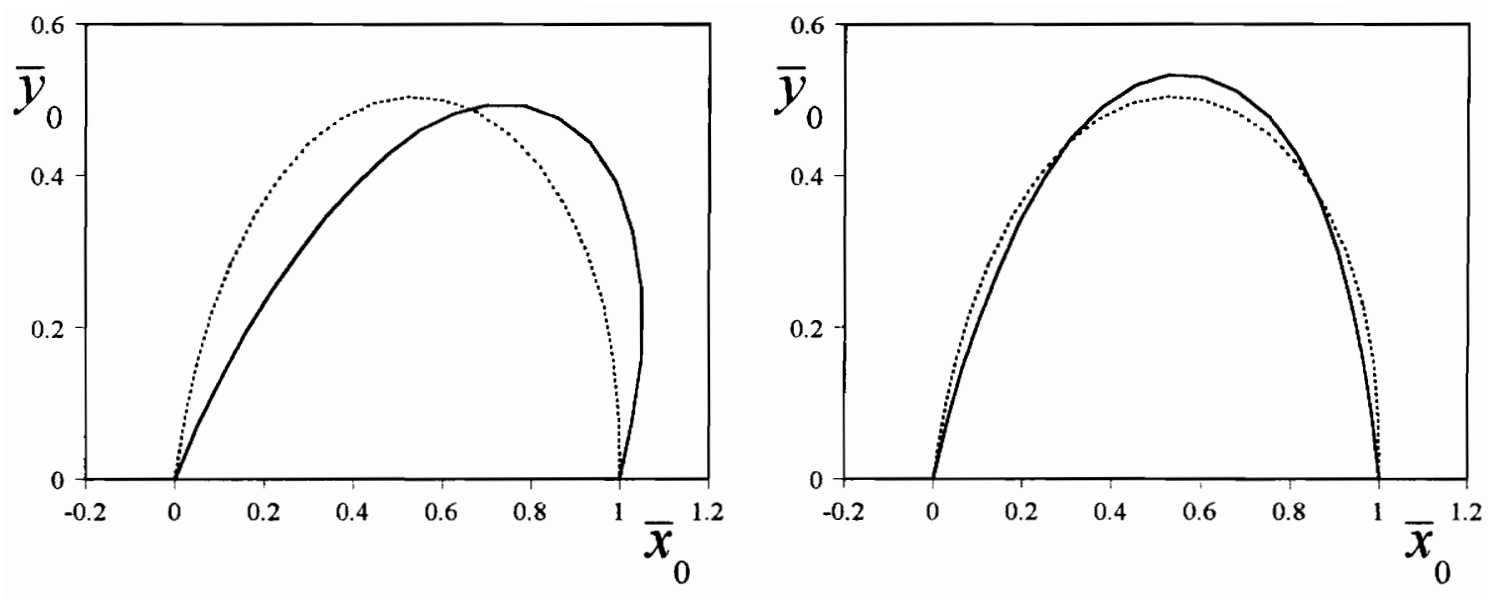

$$
\bar{\tau}=T_{1}
$$

$$
\bar{\tau}=\frac{5}{4} T_{1}
$$
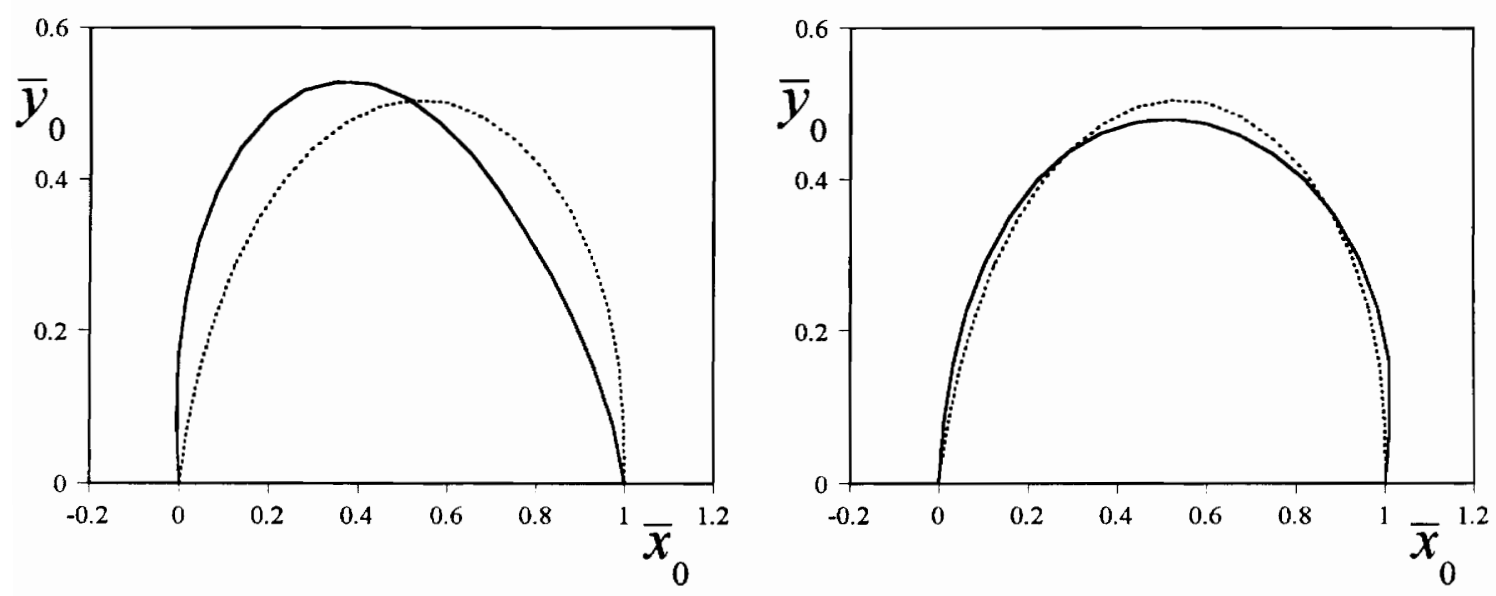

$$
\bar{\tau}=\frac{3}{2} T_{1}
$$

$$
\bar{\tau}=\frac{7}{4} T_{1}
$$

Figure 4.8 Example 4.5.2, Mode 1, Displacement with Time (Second Cycle) 


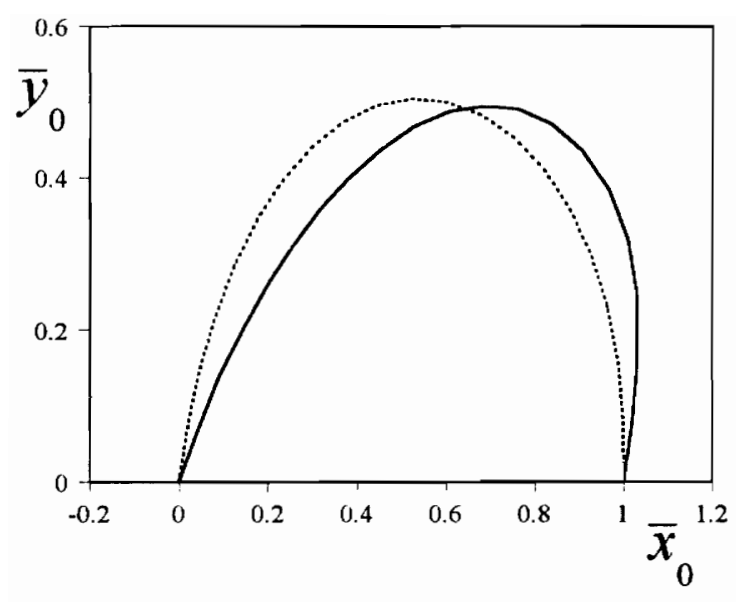

$$
\bar{\tau}=2 T_{1}
$$

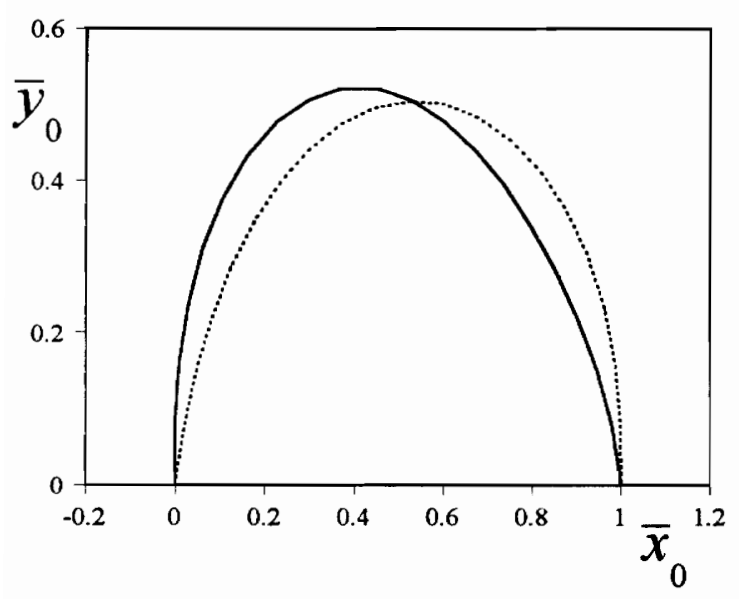

$$
\bar{\tau}=\frac{5}{2} T_{1}
$$

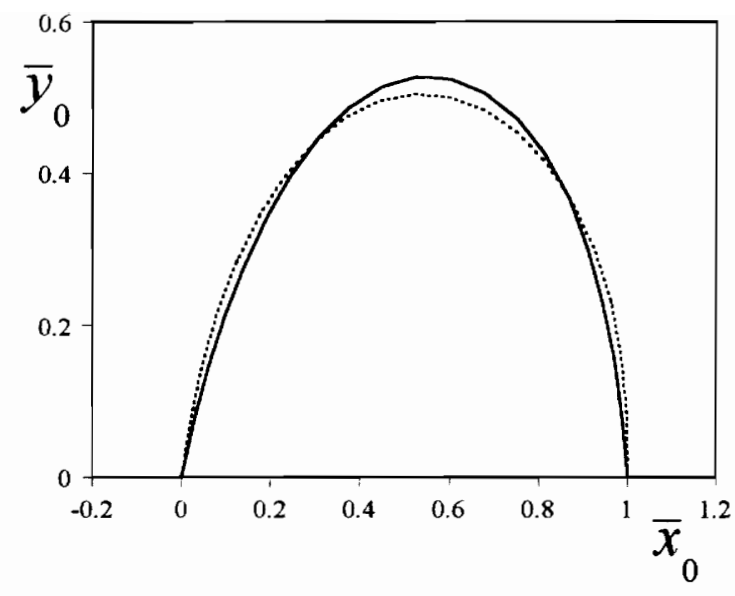

$\bar{\tau}=\frac{9}{4} T_{1}$

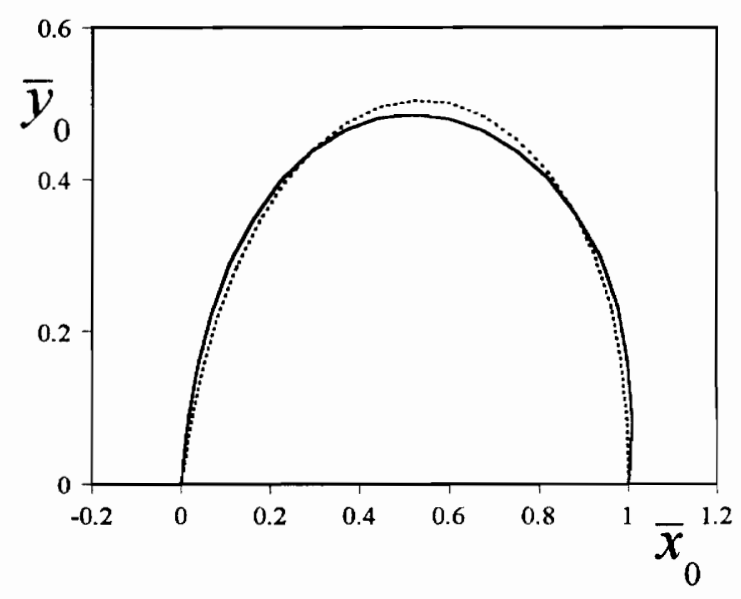

$$
\bar{\tau}=\frac{11}{4} T_{1}
$$

Figure 4.9 Example 4.5.2, Mode 1, Displacement with Time (Third Cycle) 

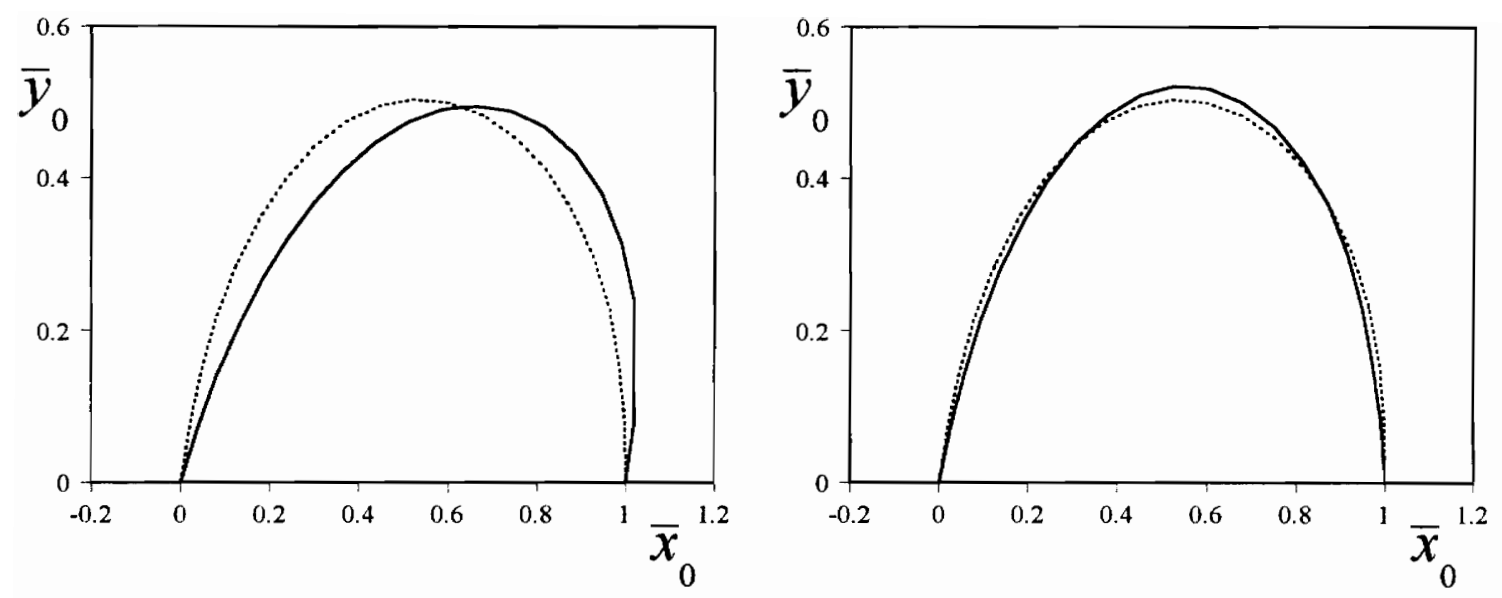

$\bar{\tau}=3 T_{1}$

$$
\bar{\tau}=\frac{13}{4} T_{1}
$$
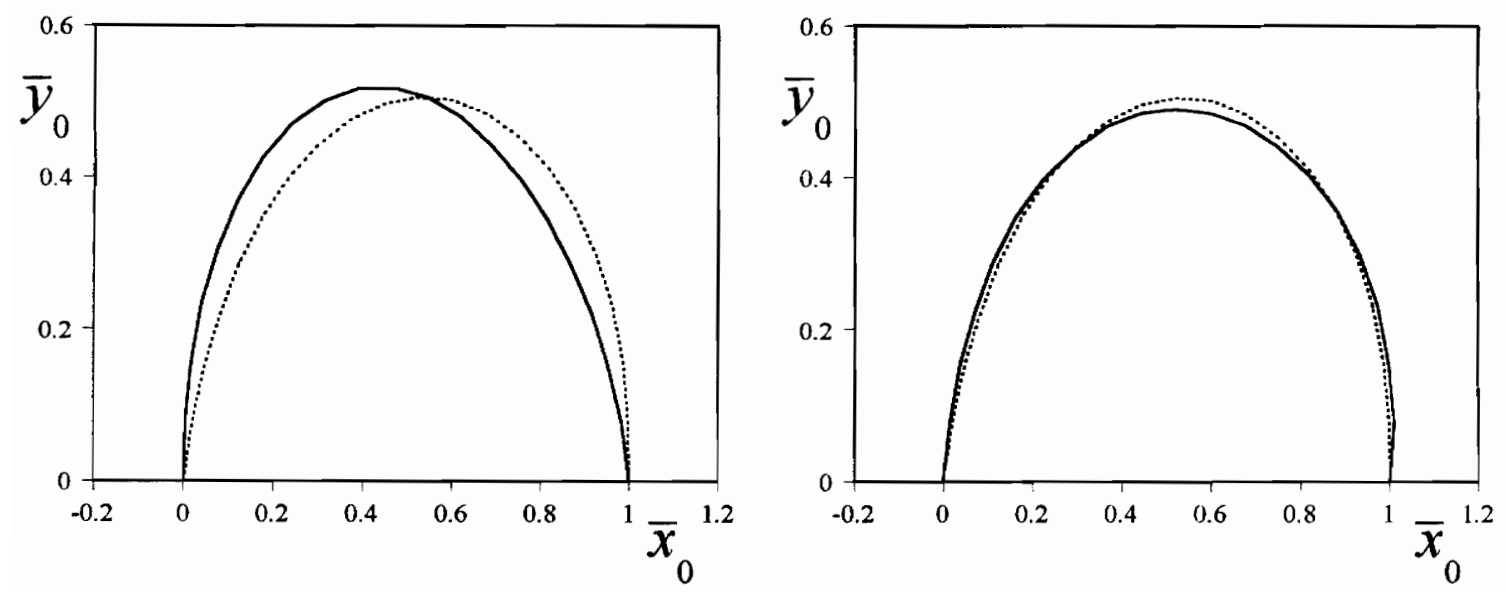

$$
\bar{\tau}=\frac{7}{2} T_{1}
$$

$$
\bar{\tau}=\frac{15}{4} T_{1}
$$

Figure 4.10 Example 4.5.2, Mode 1, Displacement with Time (Fourth Cycle) 

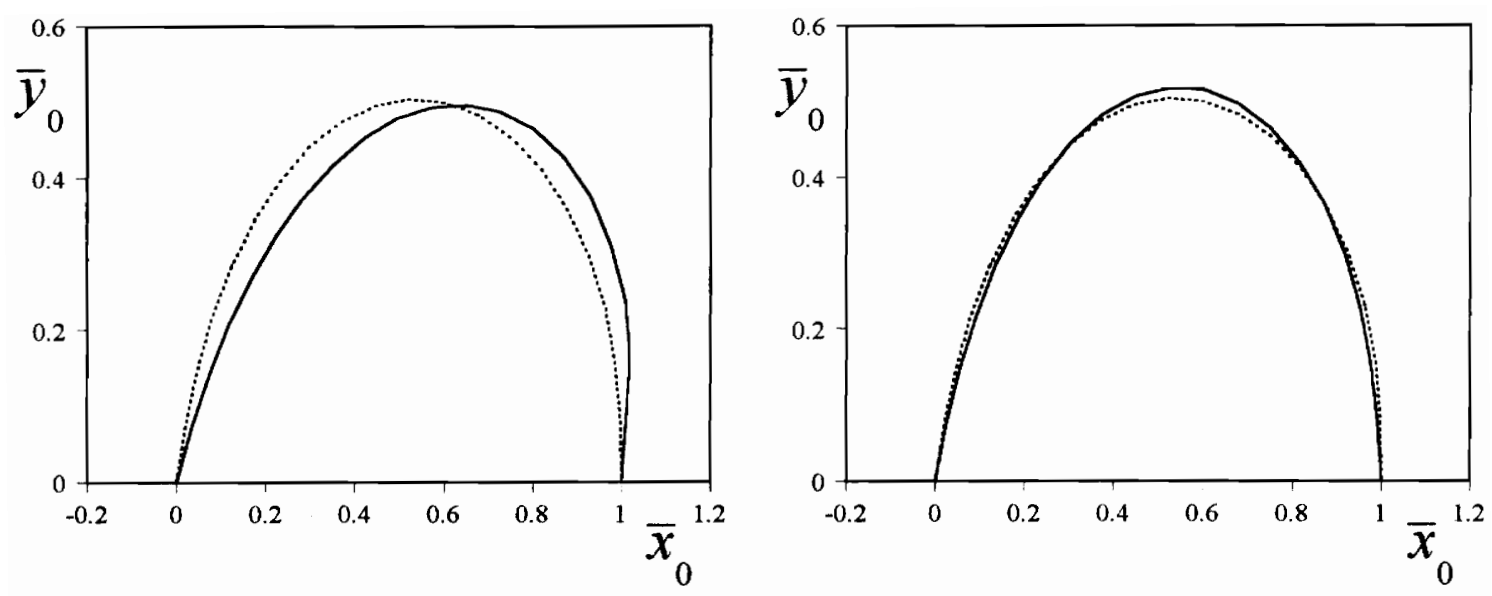

$$
\bar{\tau}=4 T_{1}
$$

$$
\tau=\frac{17}{4} T_{1}
$$
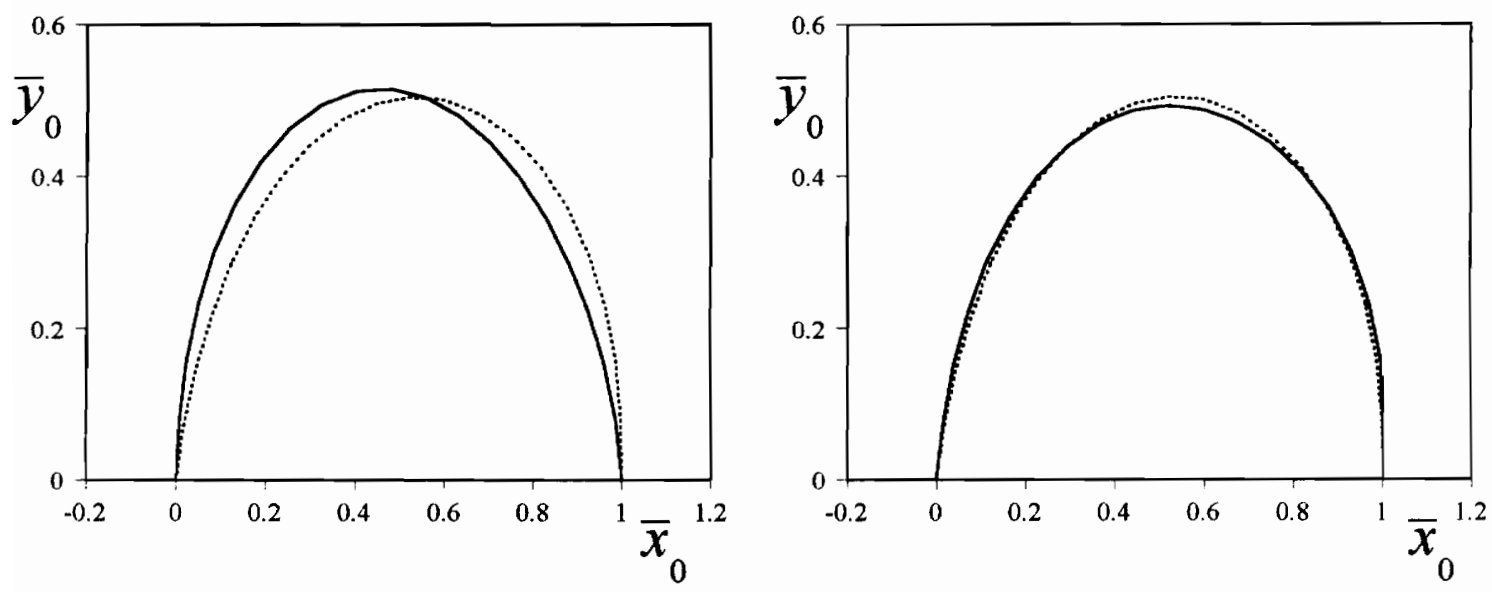

$$
\bar{\tau}=\frac{9}{2} T_{1}
$$

$$
\bar{\tau}=\frac{19}{4} T_{1}
$$

Figure 4.11 Example 4.5.2, Mode 1, Displacement with Time (Fifth Cycle) 

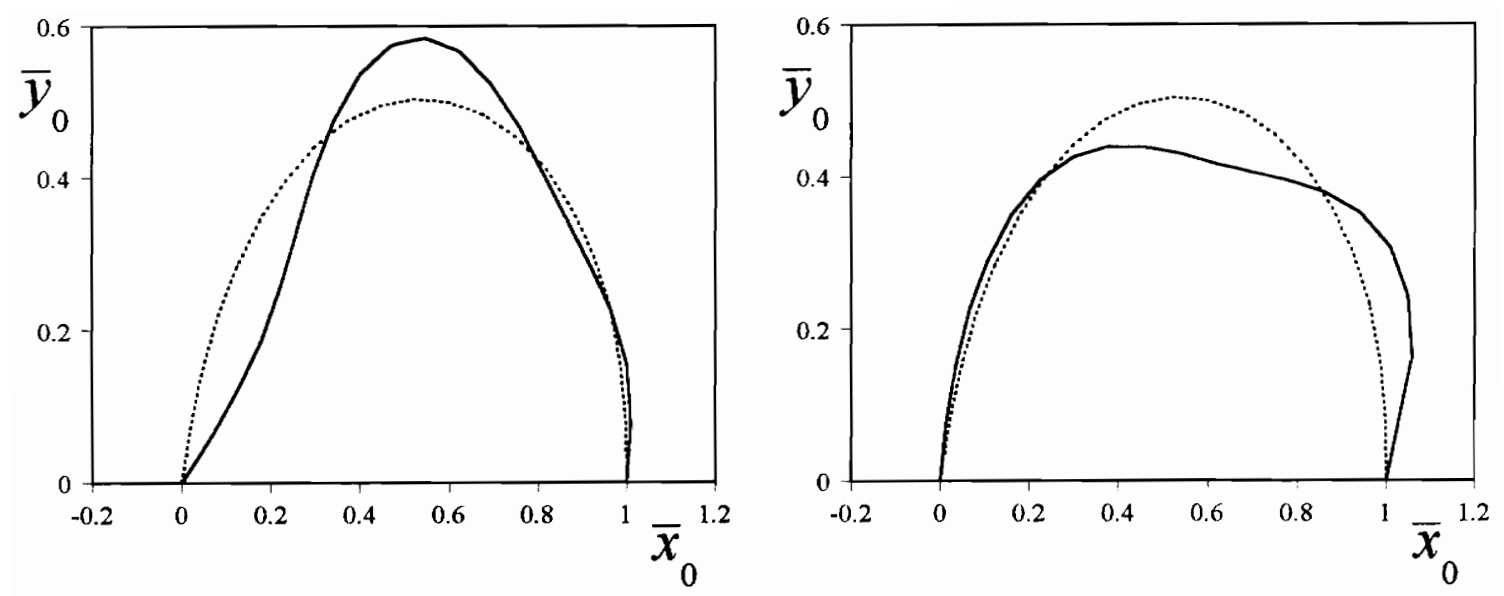

$\bar{\tau}=0$

$\bar{\tau}=\frac{1}{4} T_{2}$
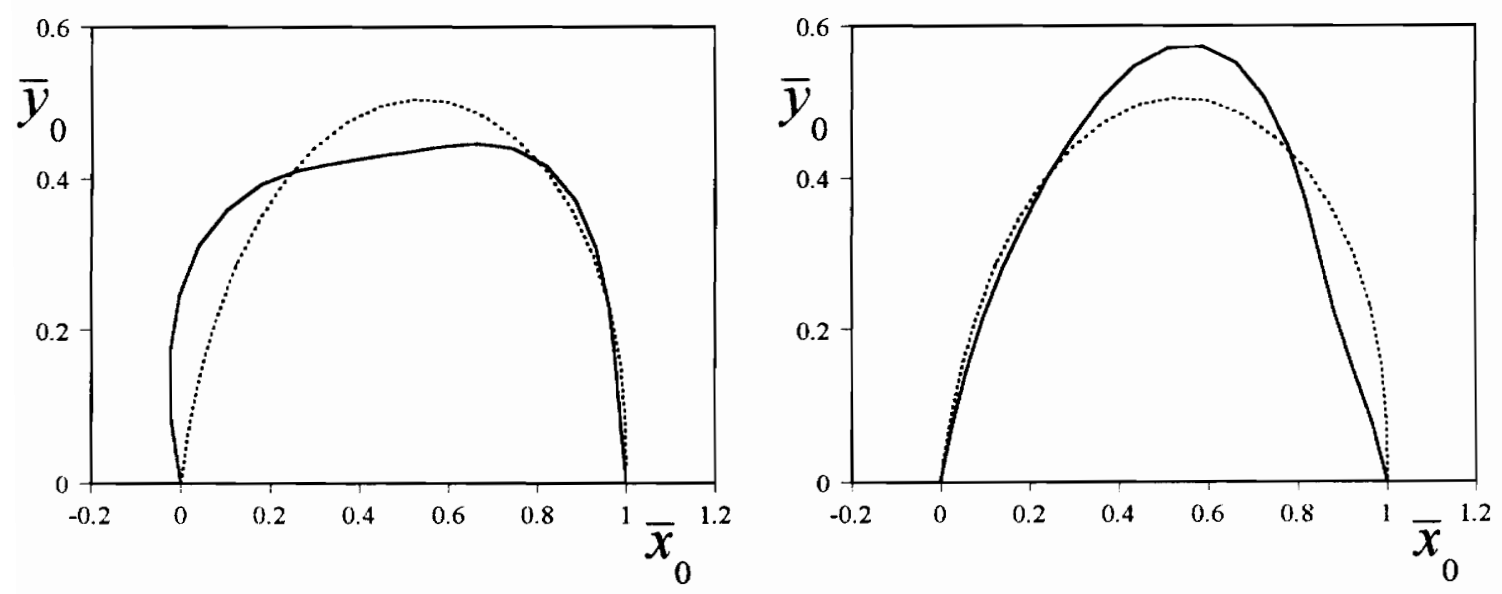

$\bar{\tau}=\frac{1}{2} T_{2}$

$\bar{\tau}=\frac{3}{4} T_{2}$

Figure 4.12 Example 4.5.2, Mode 2, Displacement with Time 

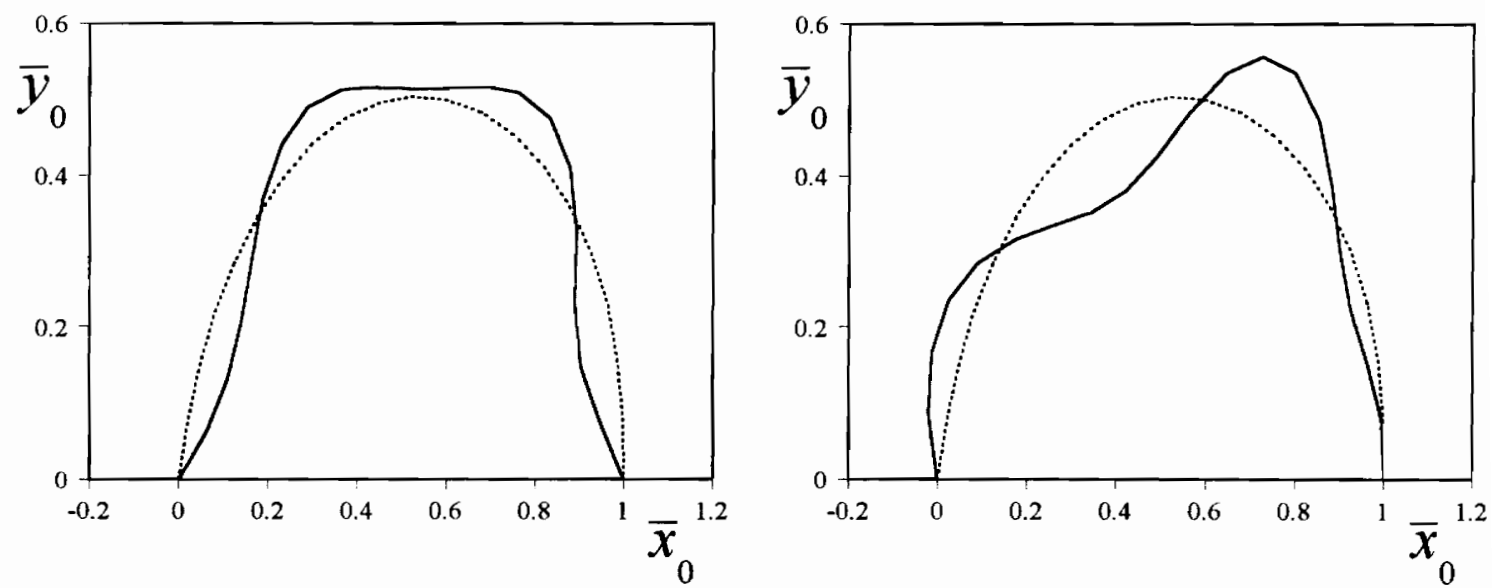

$\bar{\tau}=0$

$$
\bar{\tau}=\frac{1}{4} T_{3}
$$
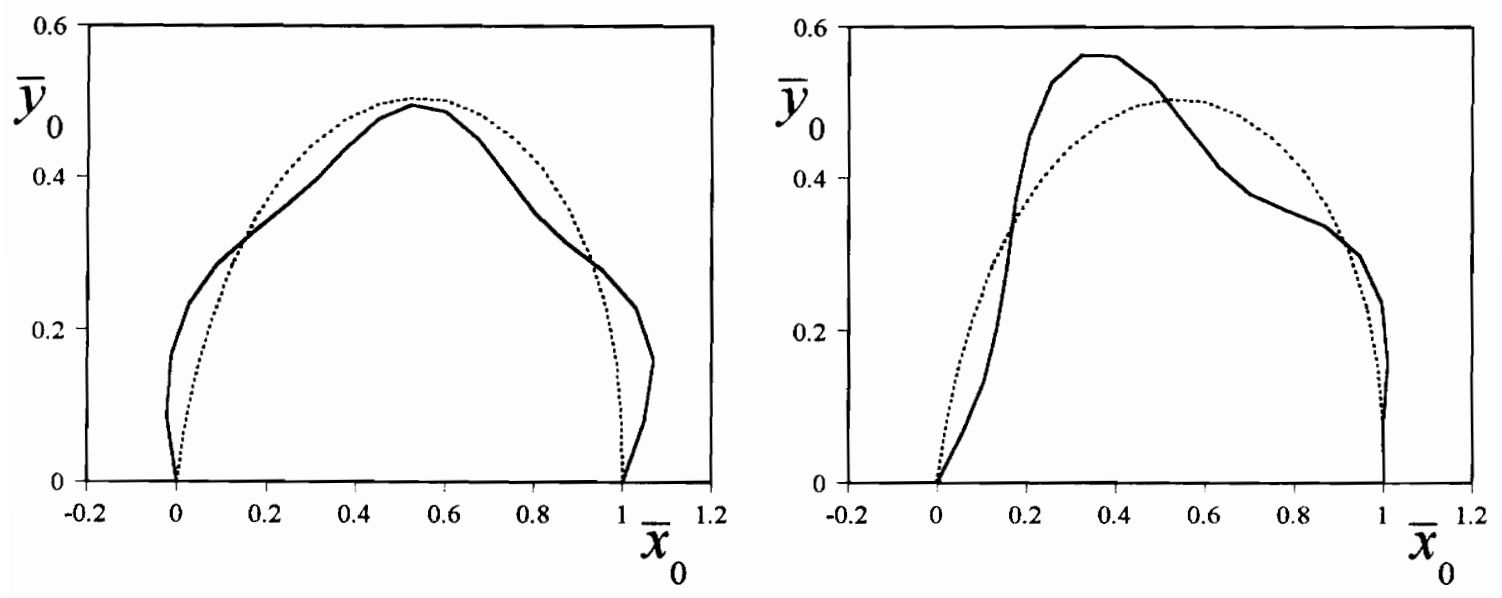

$$
\bar{\tau}=\frac{1}{2} T_{3}
$$

$$
\bar{\tau}=\frac{3}{4} T_{3}
$$

Figure 4.13 Example 4.5.2, Mode 3, Displacement with Time 

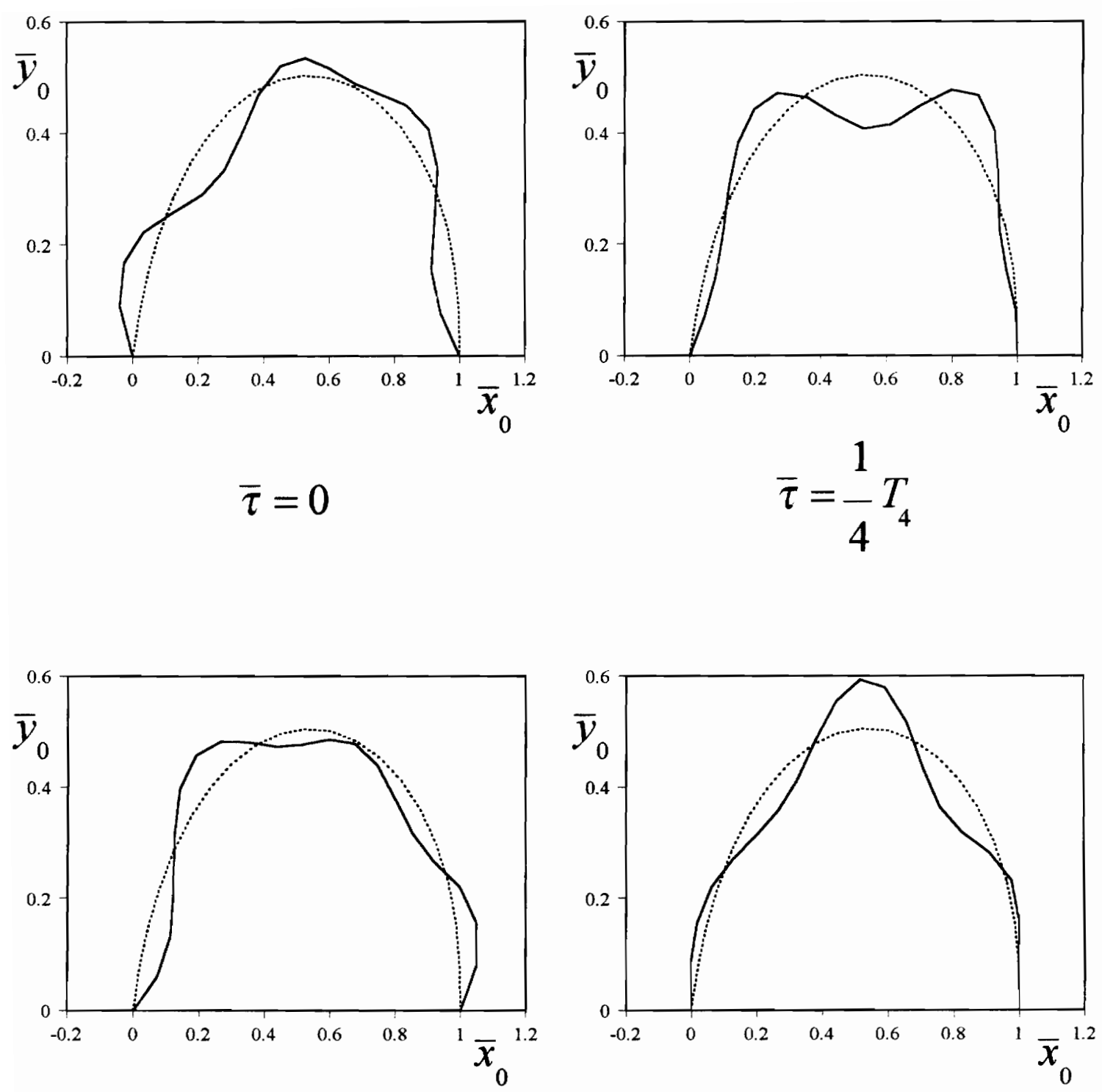

$$
\bar{\tau}=\frac{1}{2} T_{4}
$$

$$
\bar{\tau}=\frac{3}{4} T_{4}
$$

Figure 4.14 Example 4.5.2, Mode 4, Displacement with Time 


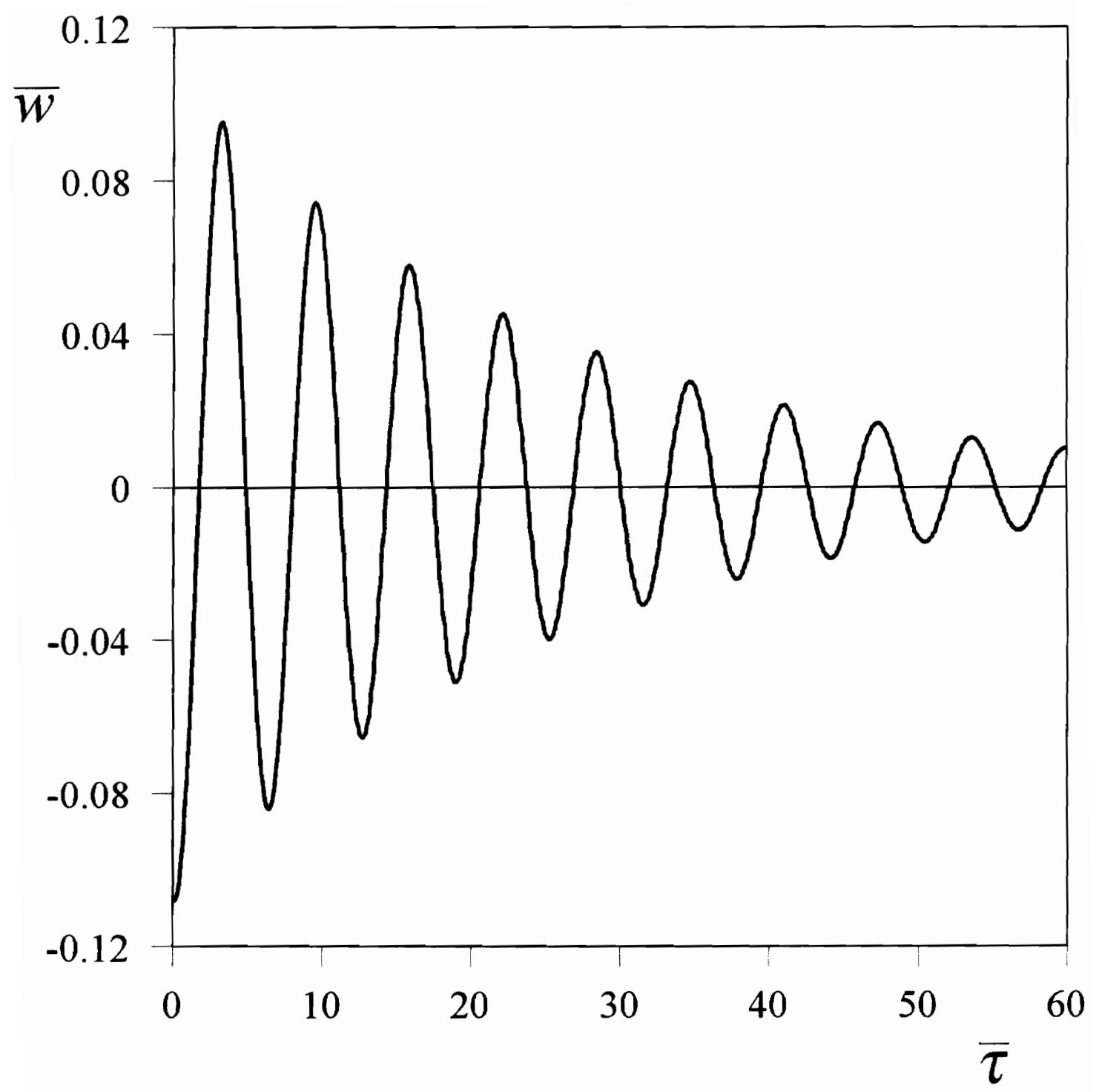

Figure 4.15 Example 4.5.2, Mode 1, Radial Displacement at Node 27 


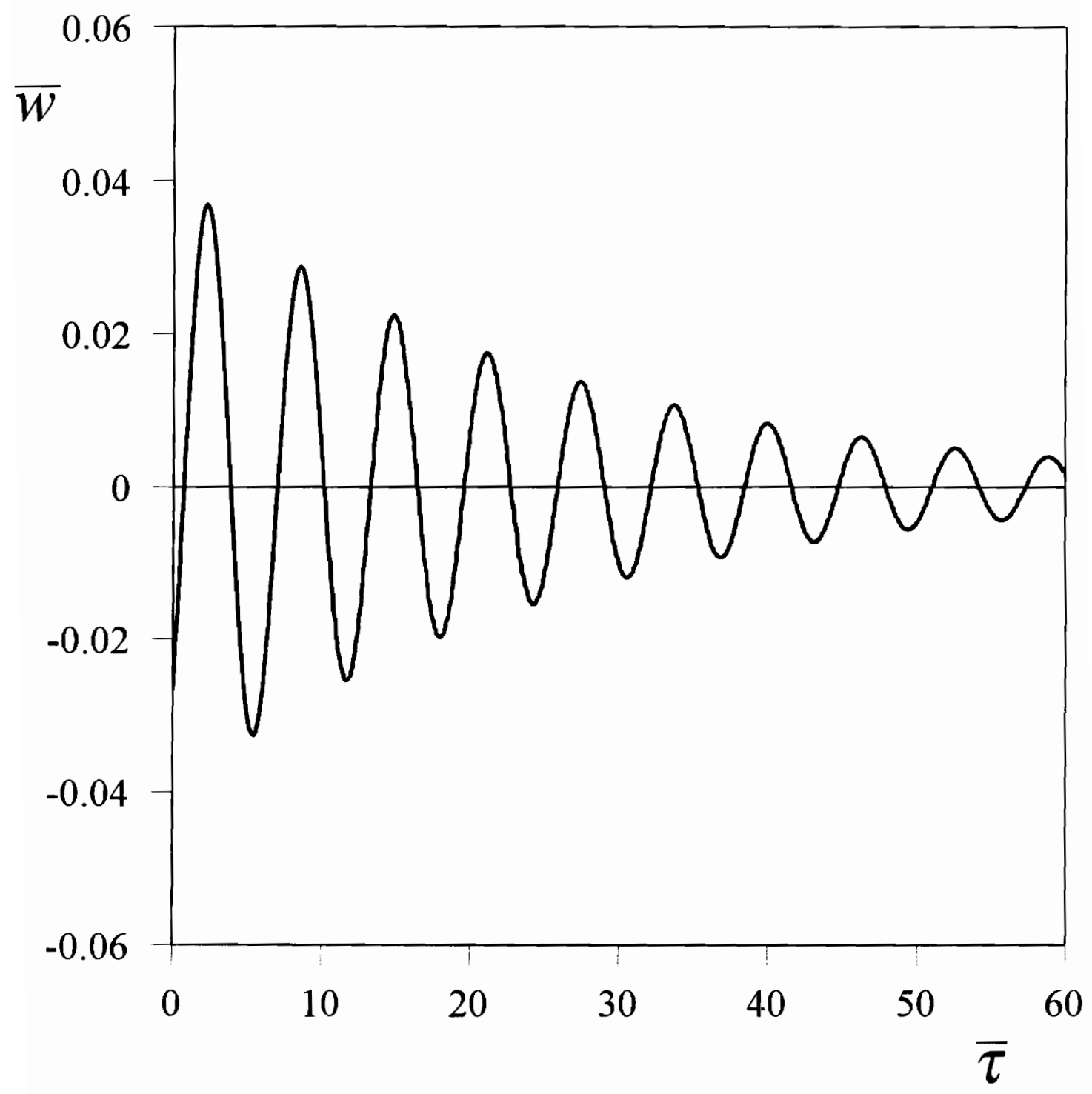

Figure 4.16 Example 4.5.2, Mode 1, Radial Displacement at Node 22 


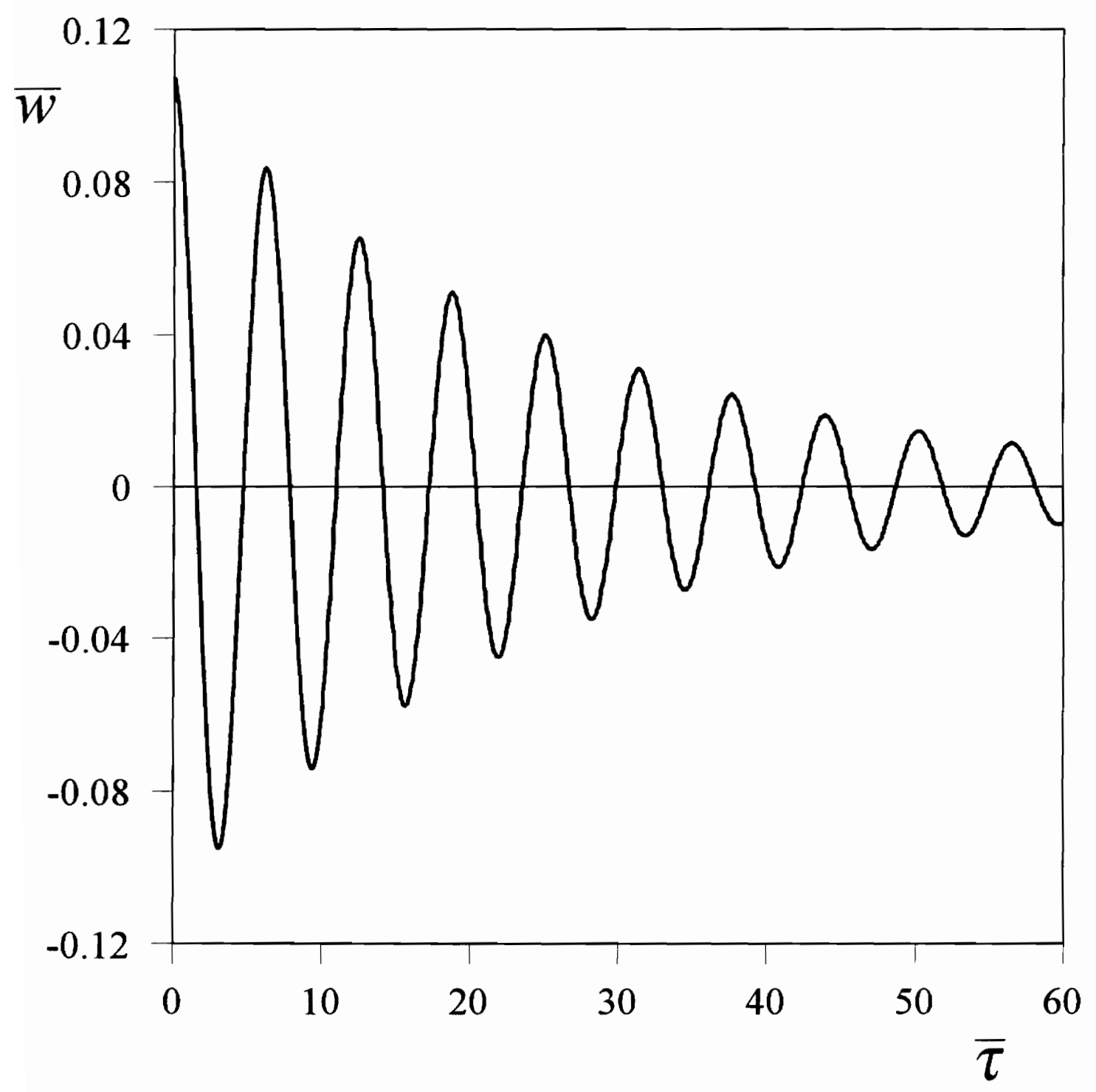

Figure 4.17 Example 4.5.2, Mode 1, Radial Displacement at Node 17 

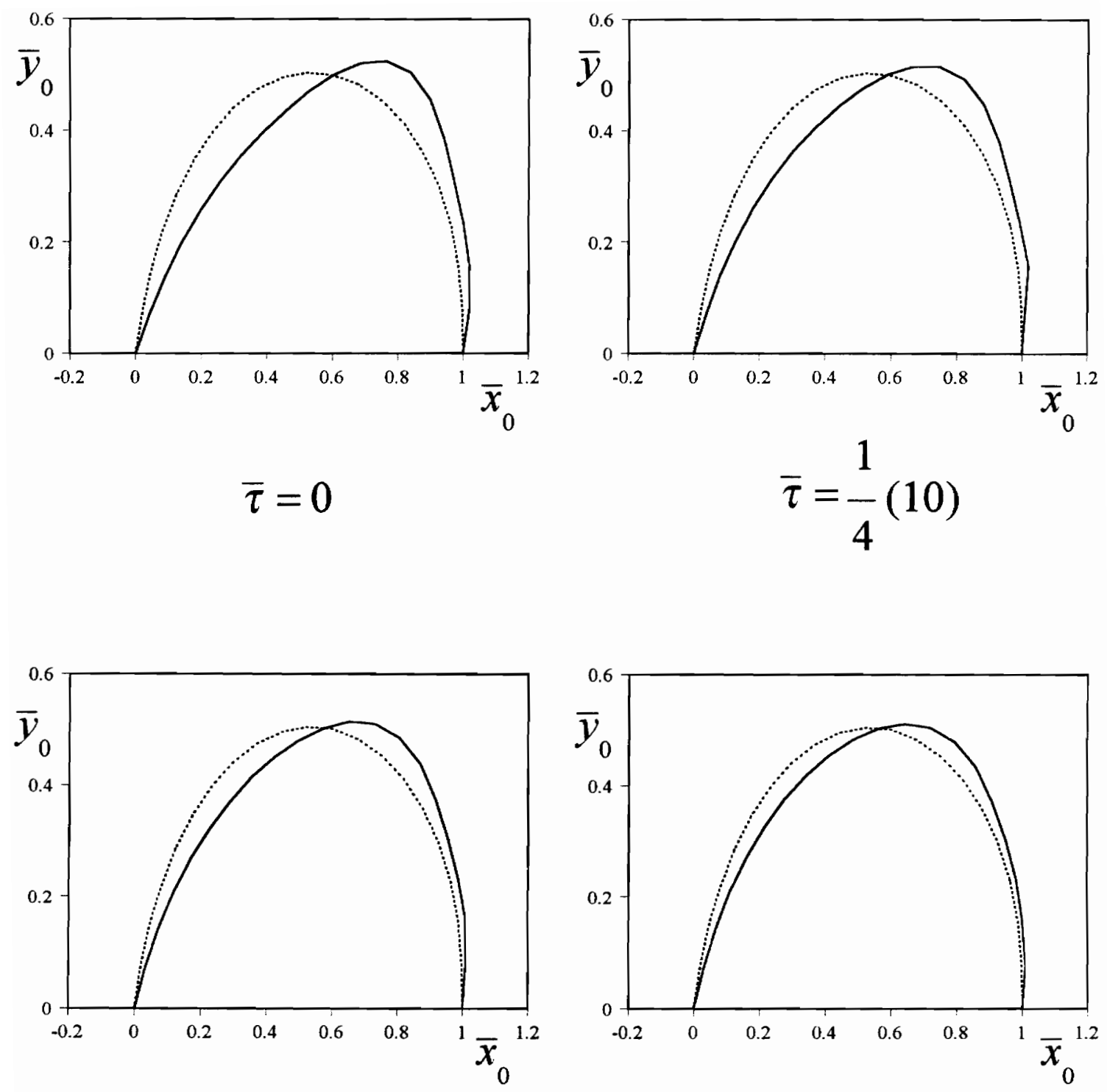

$$
\bar{\tau}=\frac{1}{2}(10)
$$$$
\bar{\tau}=\frac{3}{4}(10)
$$

Figure 4.18 Example 4.5.3, Mode 1, Displacement with Time 

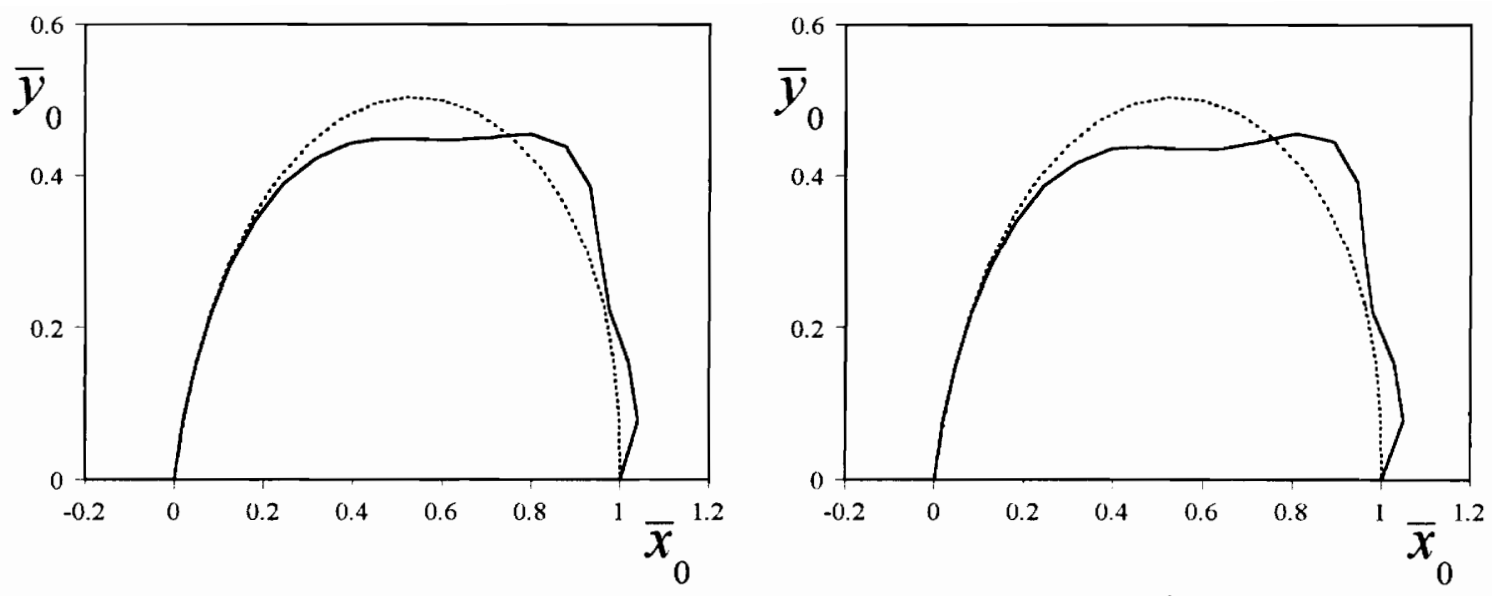

$\bar{\tau}=0$

$$
\bar{\tau}=\frac{1}{4}(2.2)
$$
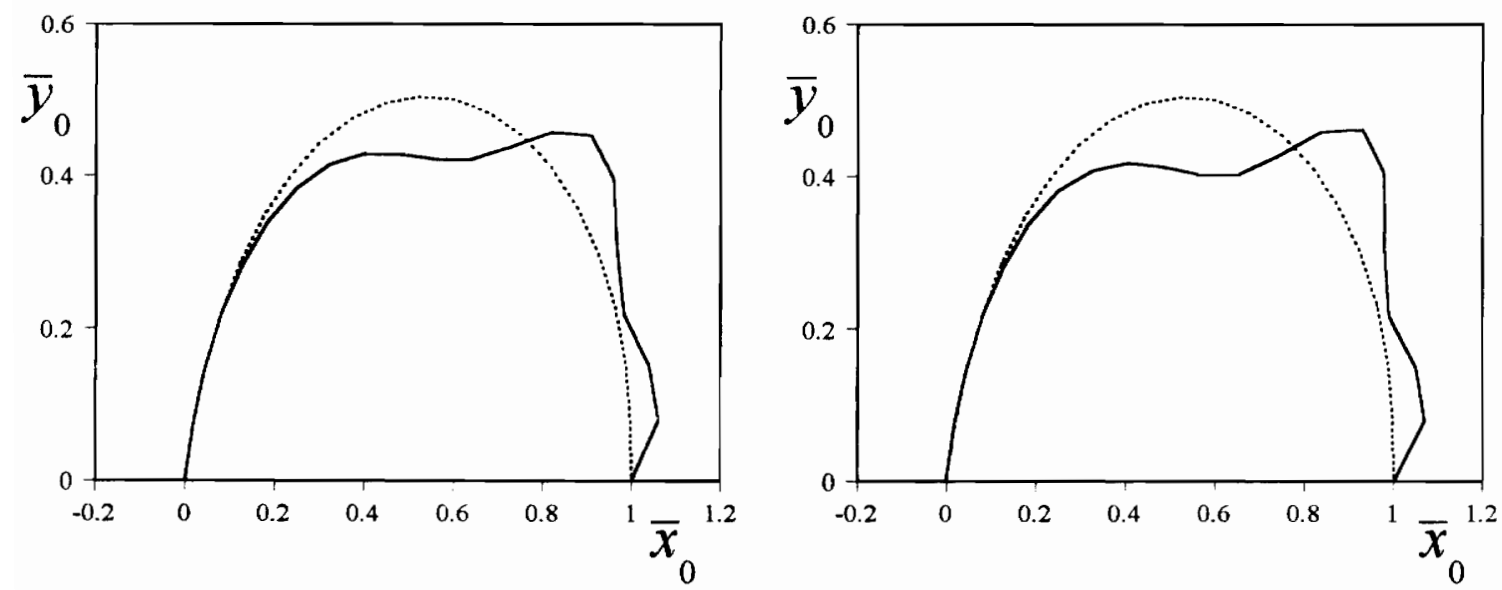

$$
\bar{\tau}=\frac{1}{2}(2.2)
$$$$
\bar{\tau}=\frac{3}{4}(2.2)
$$

Figure 4.19 Example 4.5.3, Mode 2, Displacement with Time 

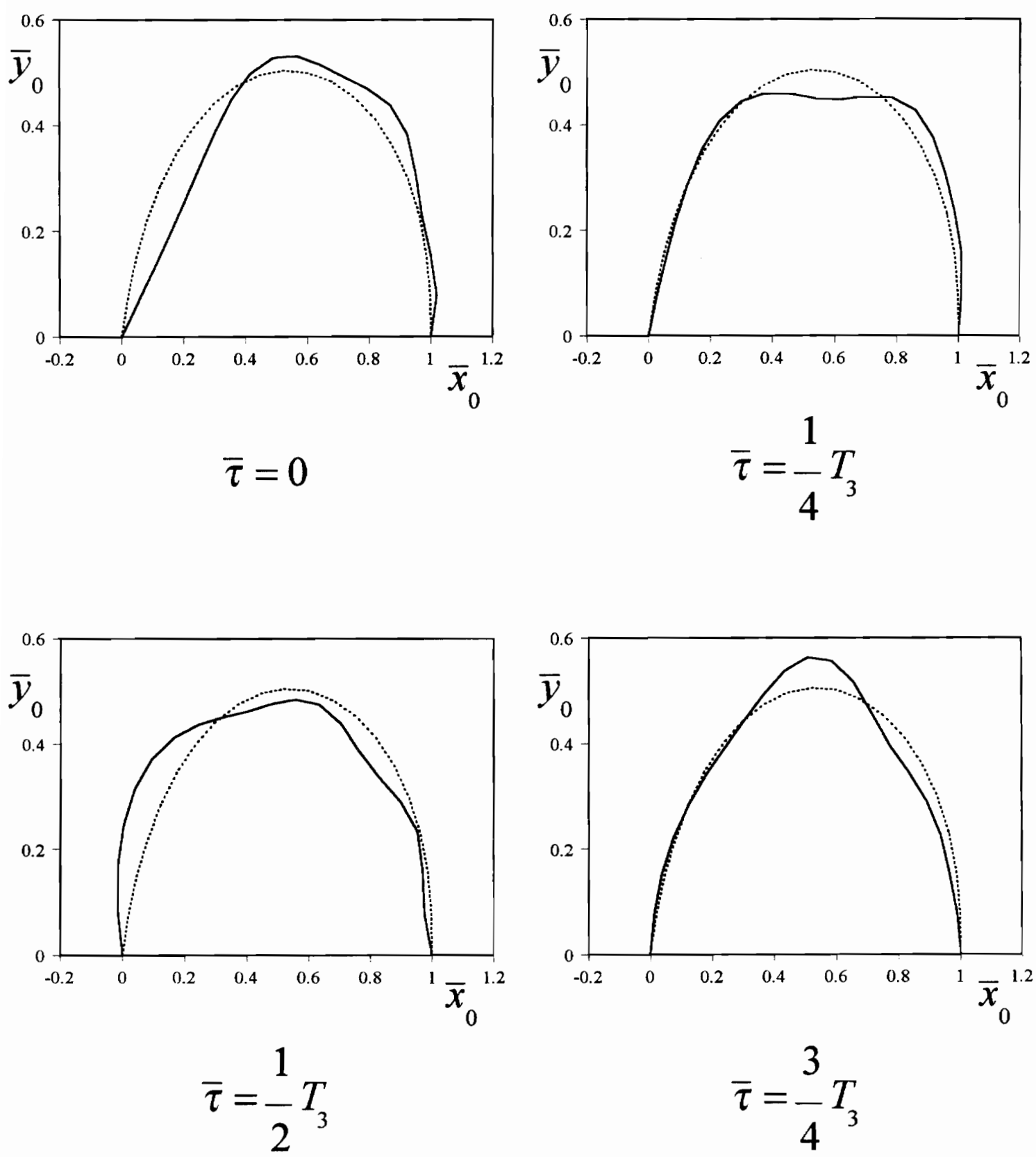

Figure 4.20 Example 4.5.3, Mode 3, Displacement with Time 

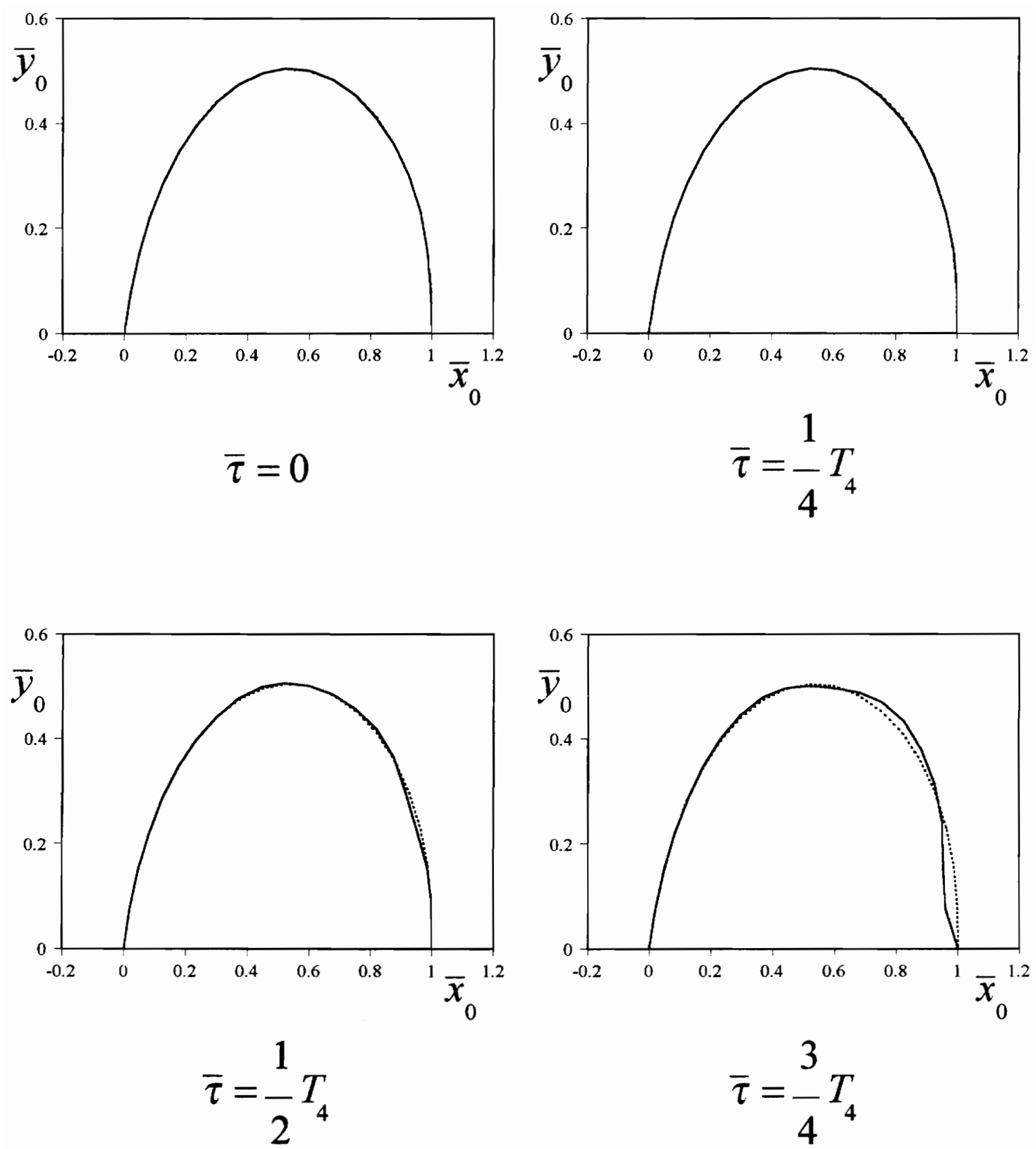

Figure 4.21 Example 4.5.3, Mode 4, Displacement with Time 

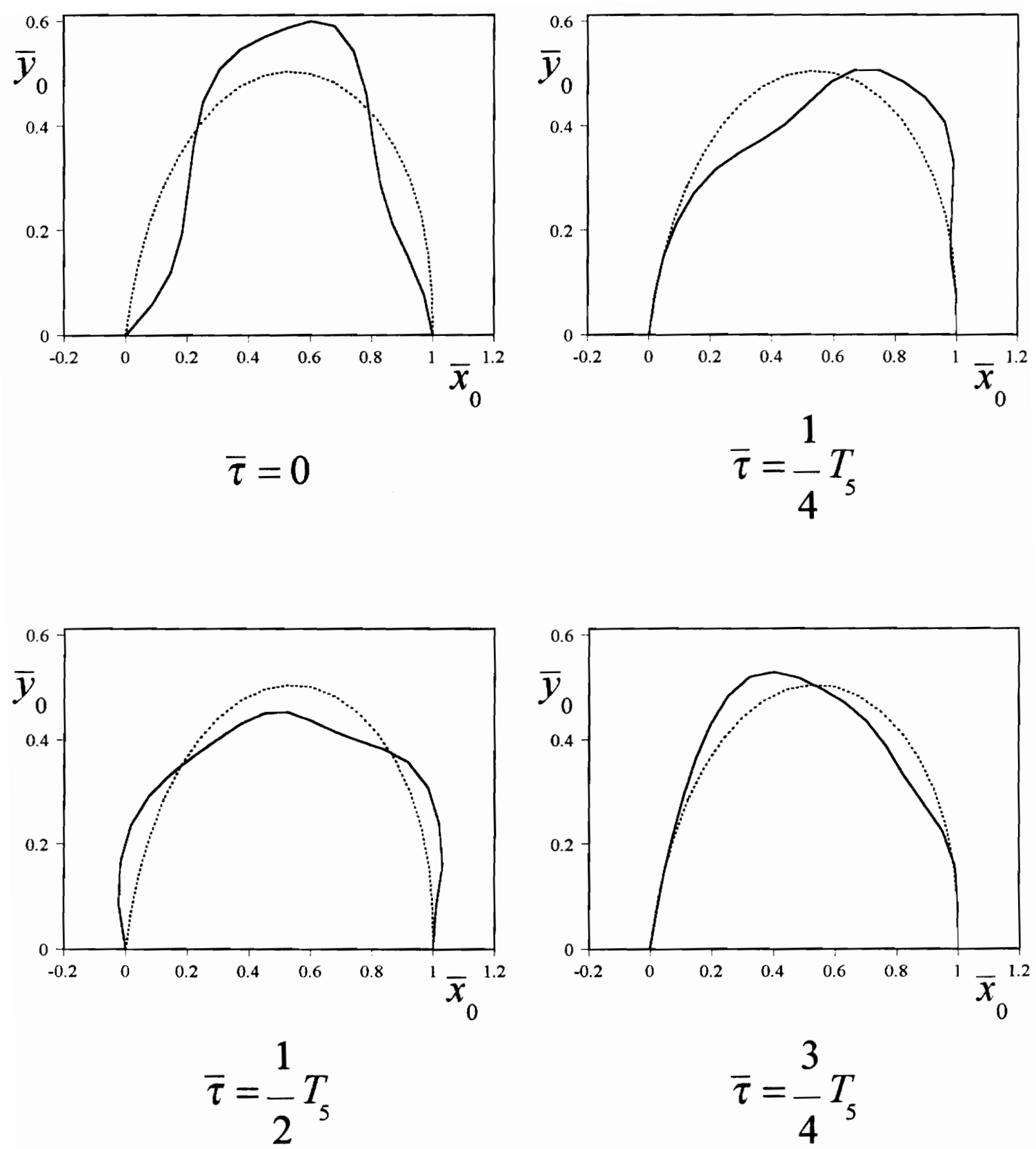

Figure 4.22 Example 4.5.3, Mode 5, Displacement with Time 


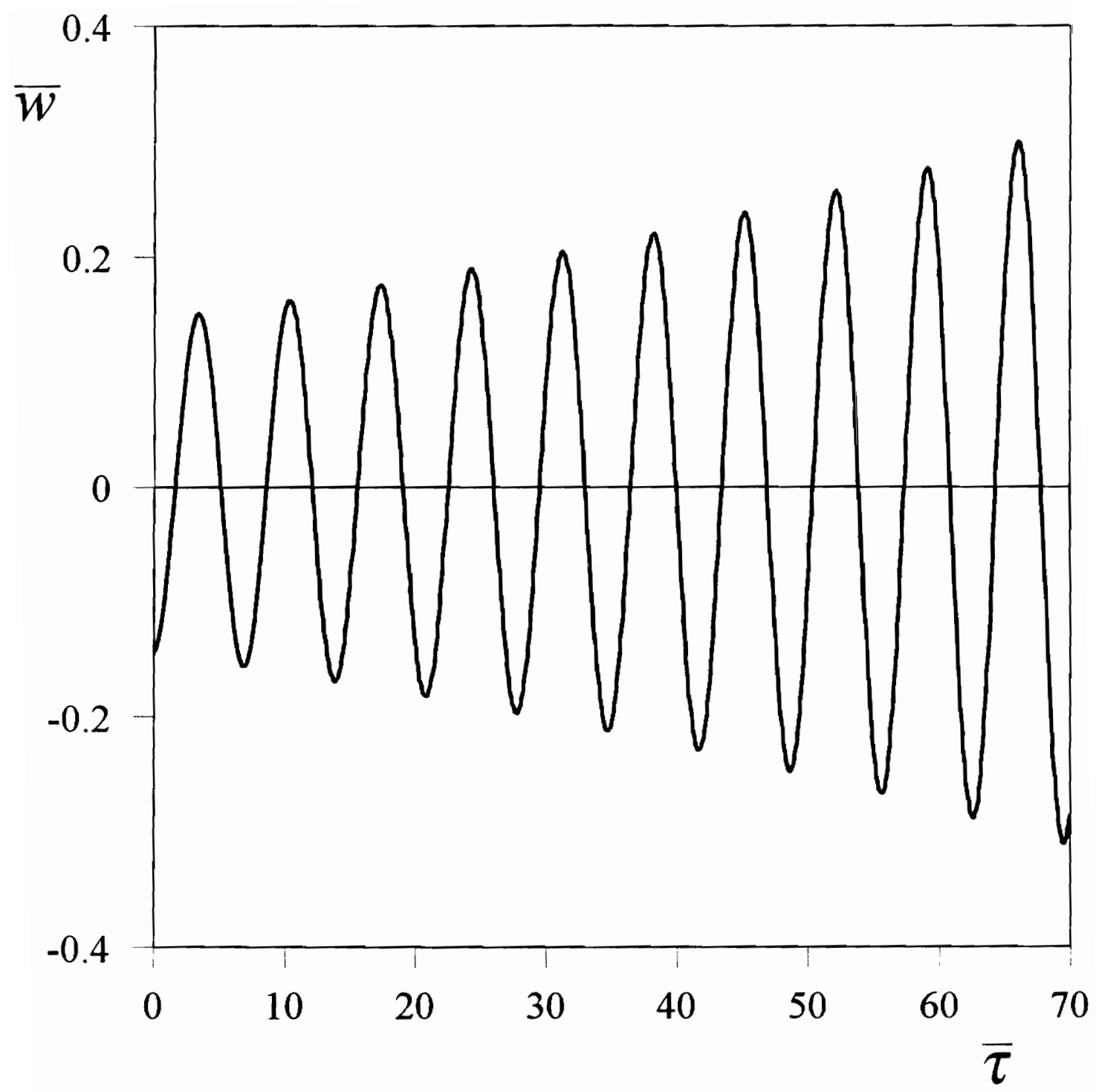

Figure 4.23 Example 4.5.3, Mode 3, Radial Displacement at Node 27 


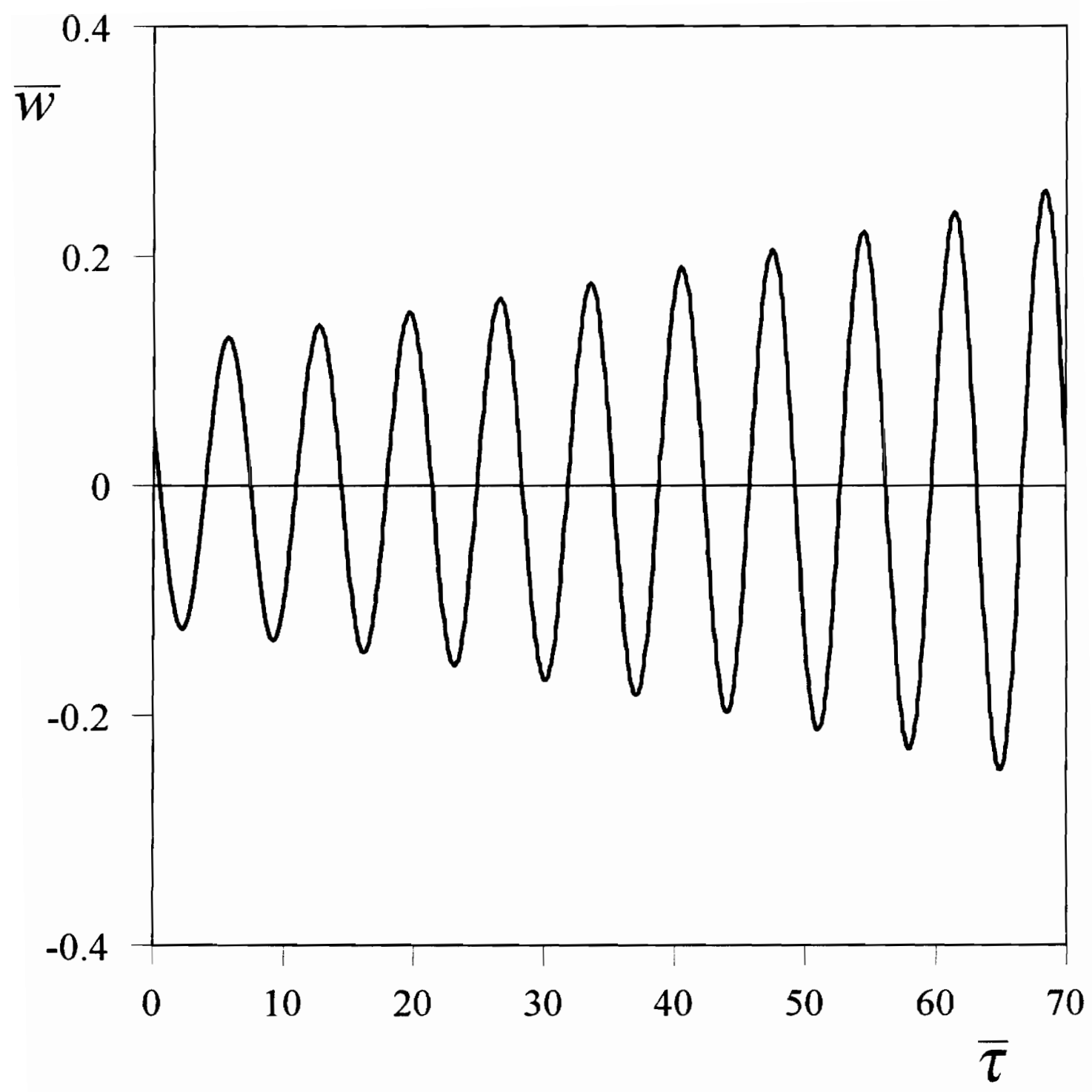

Figure 4.24 Example 4.5.3, Mode 3, Radial Displacement at Node 22 


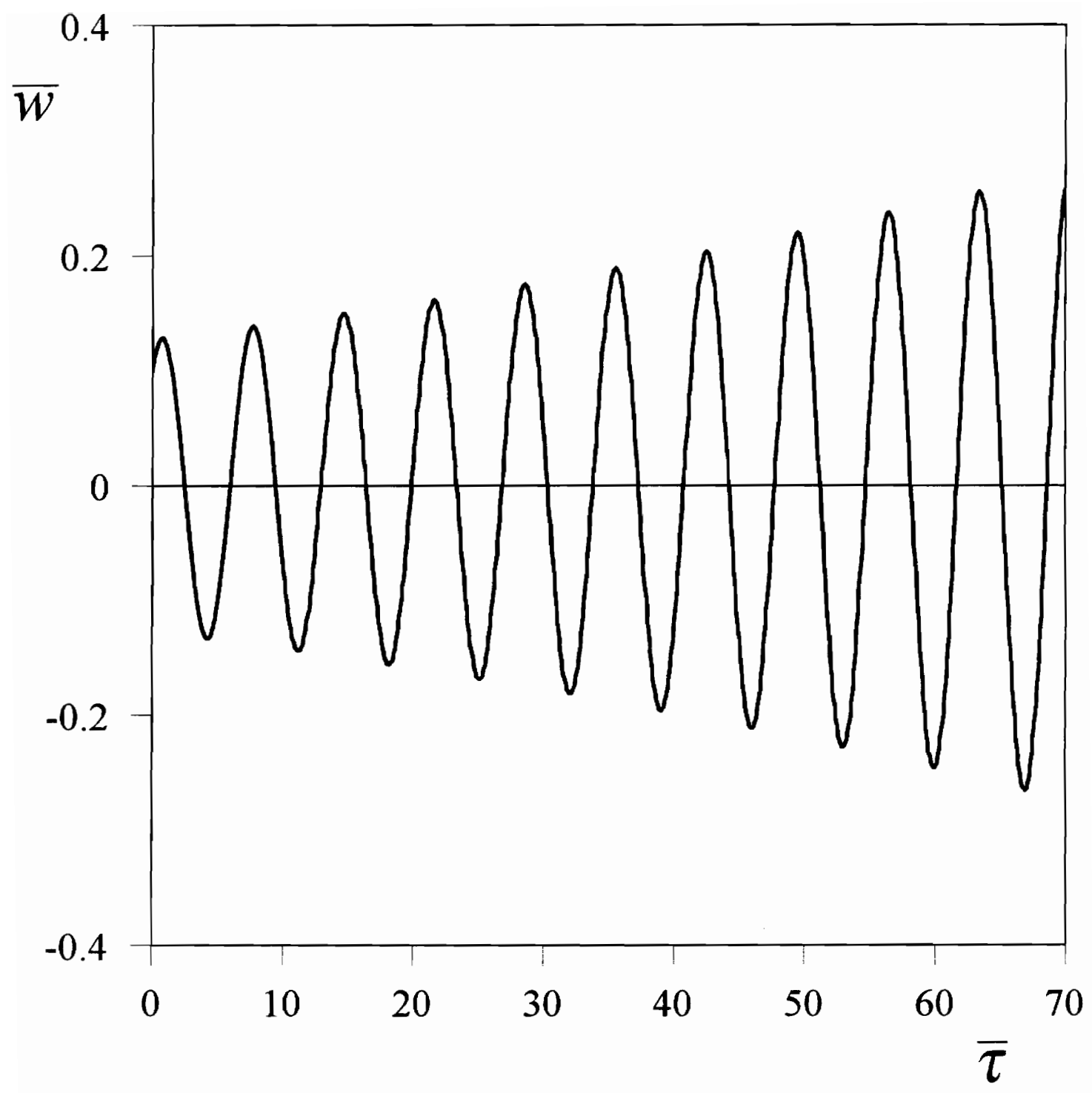

Figure 4.25 Example 4.5.3, Mode 3, Radial Displacement at Node 17 


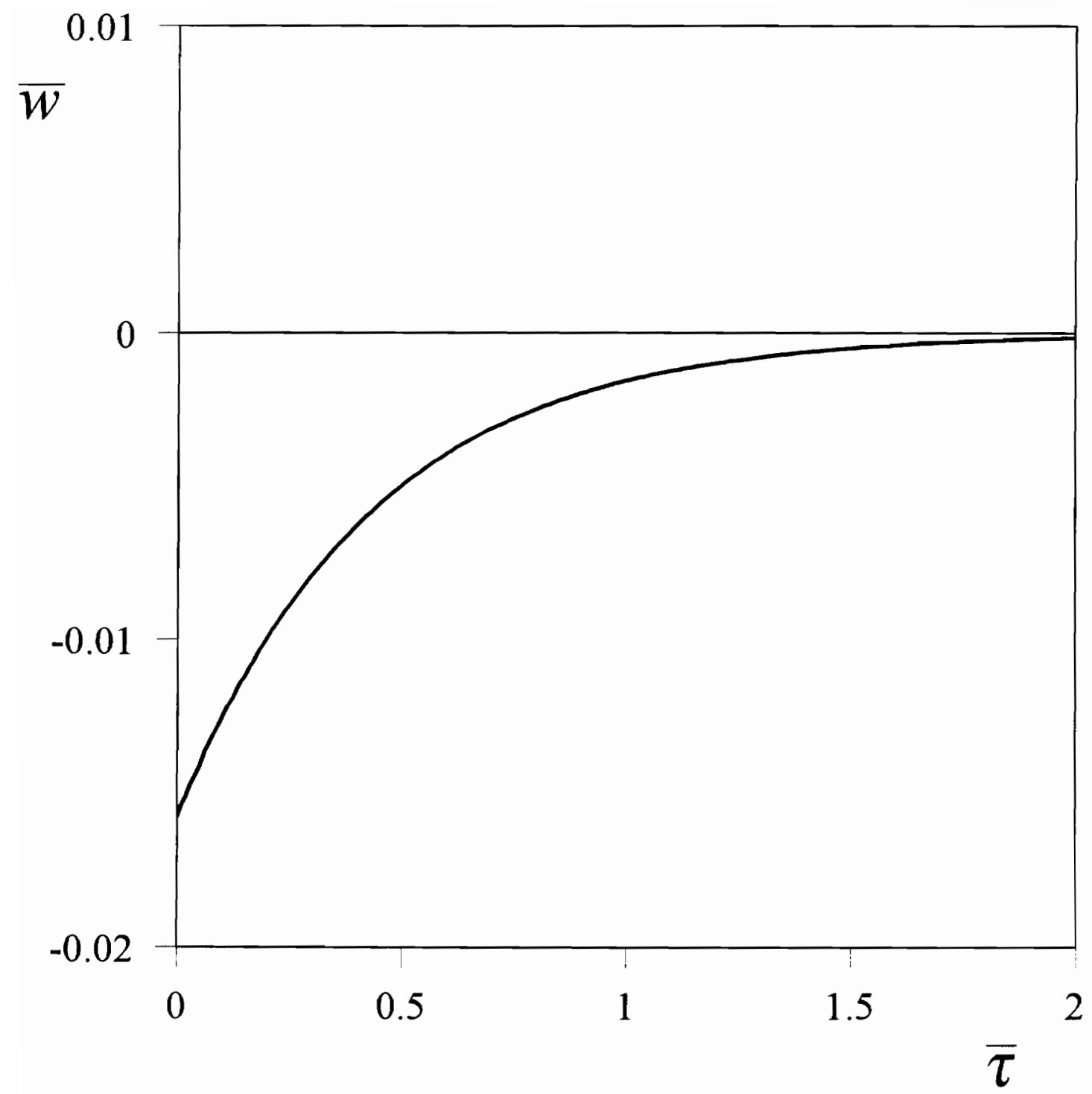

Figure 4.26 Example 4.5.3, Mode 1, Radial Displacement at Node 22 


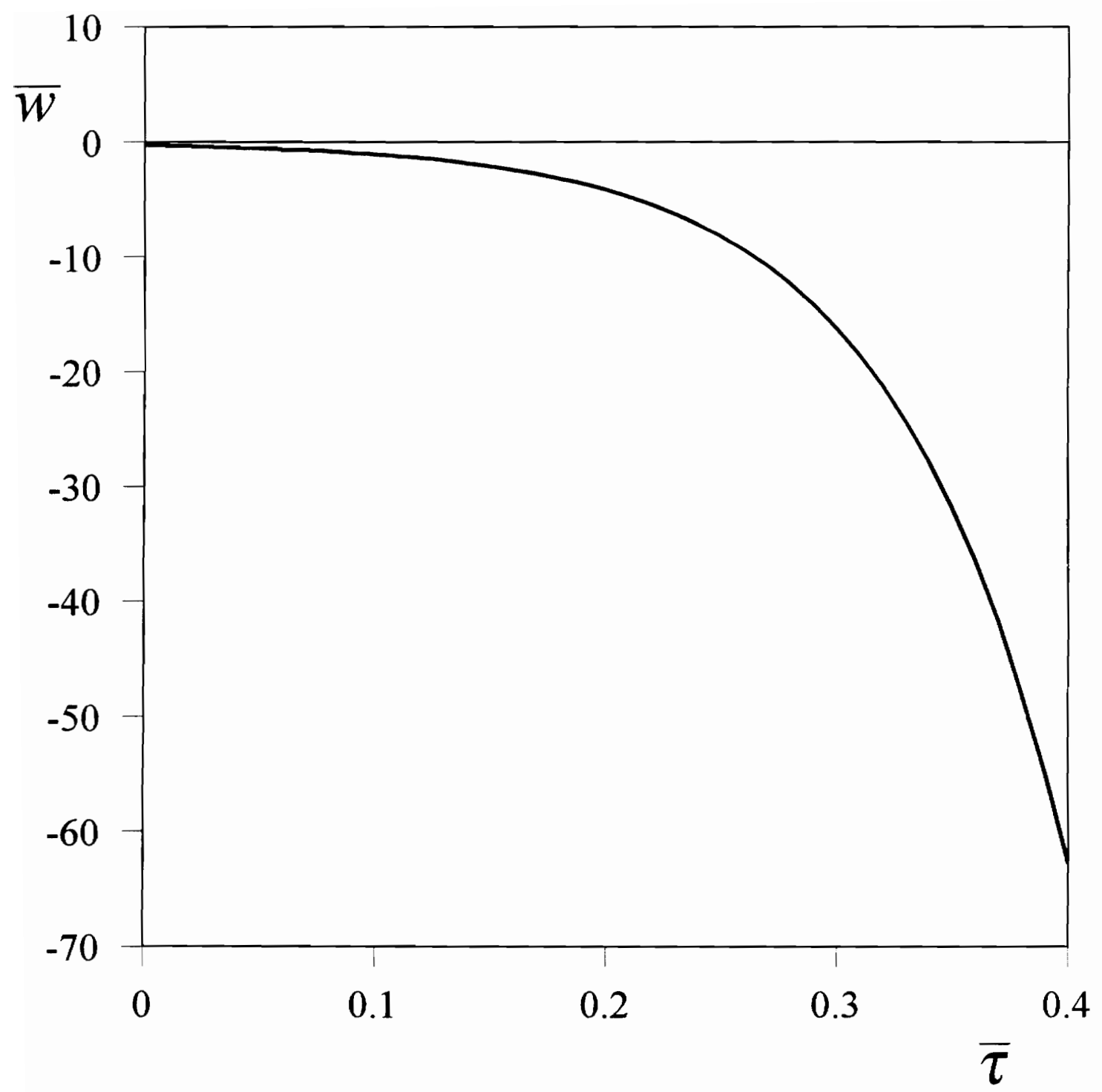

Figure 4.27 Example 4.5.3, Mode 2, Radial Displacement at Node 22 


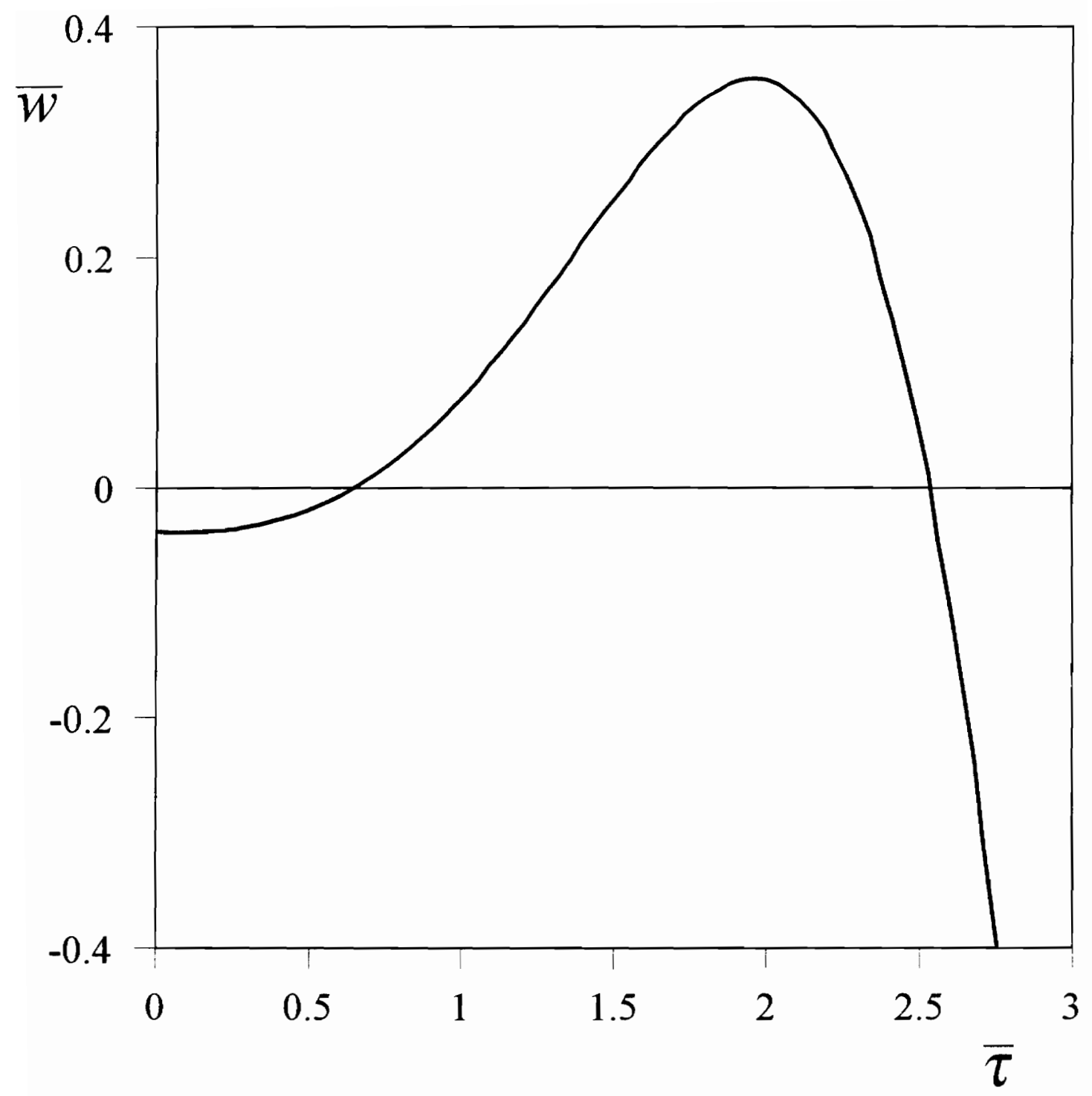

Figure 4.28 Example 4.5.3, Mode 4, Radial Displacement at Node 22 


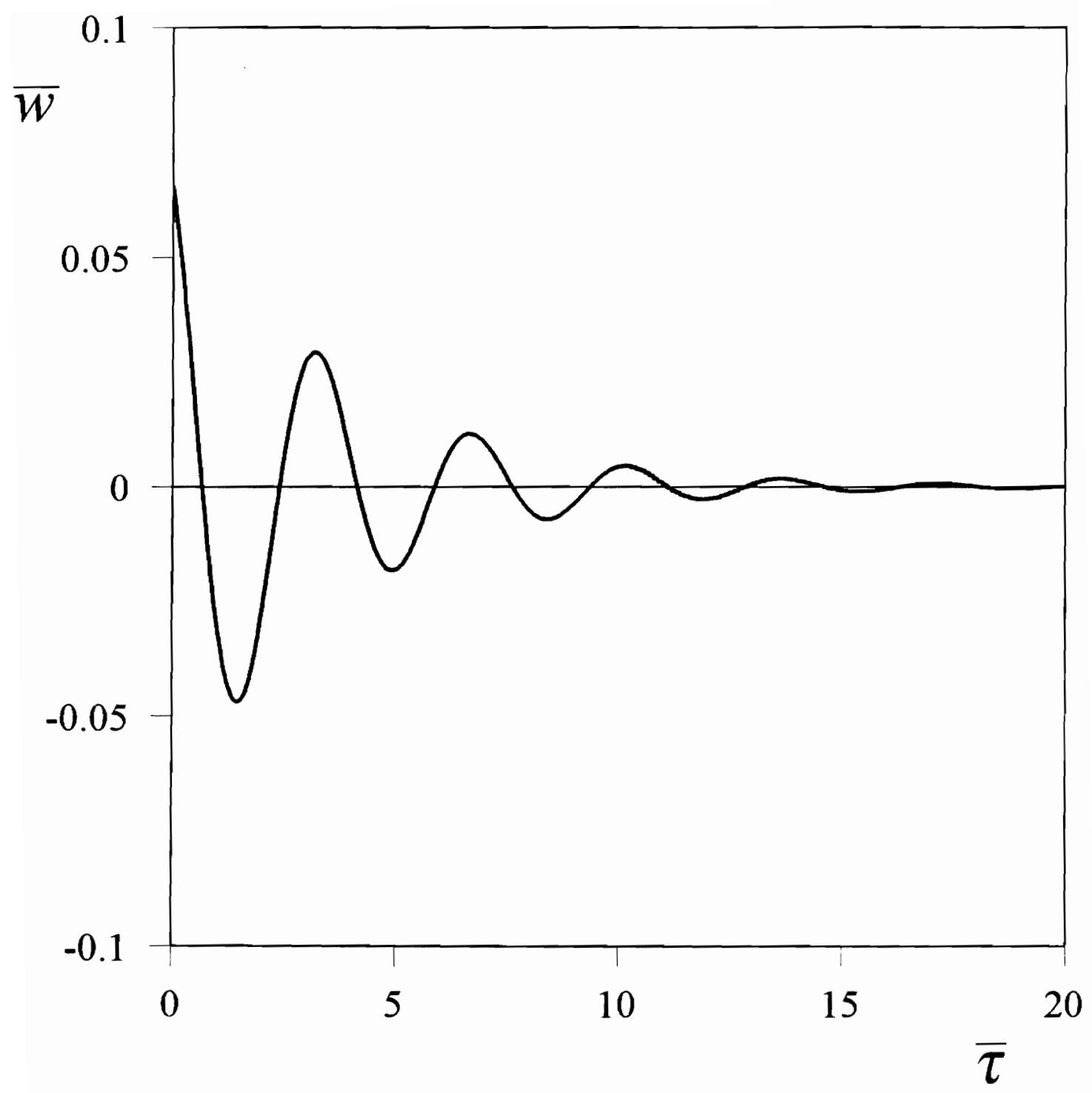

Figure 4.29 Example 4.5.3, Mode 5, Radial Displacement at Node 22 


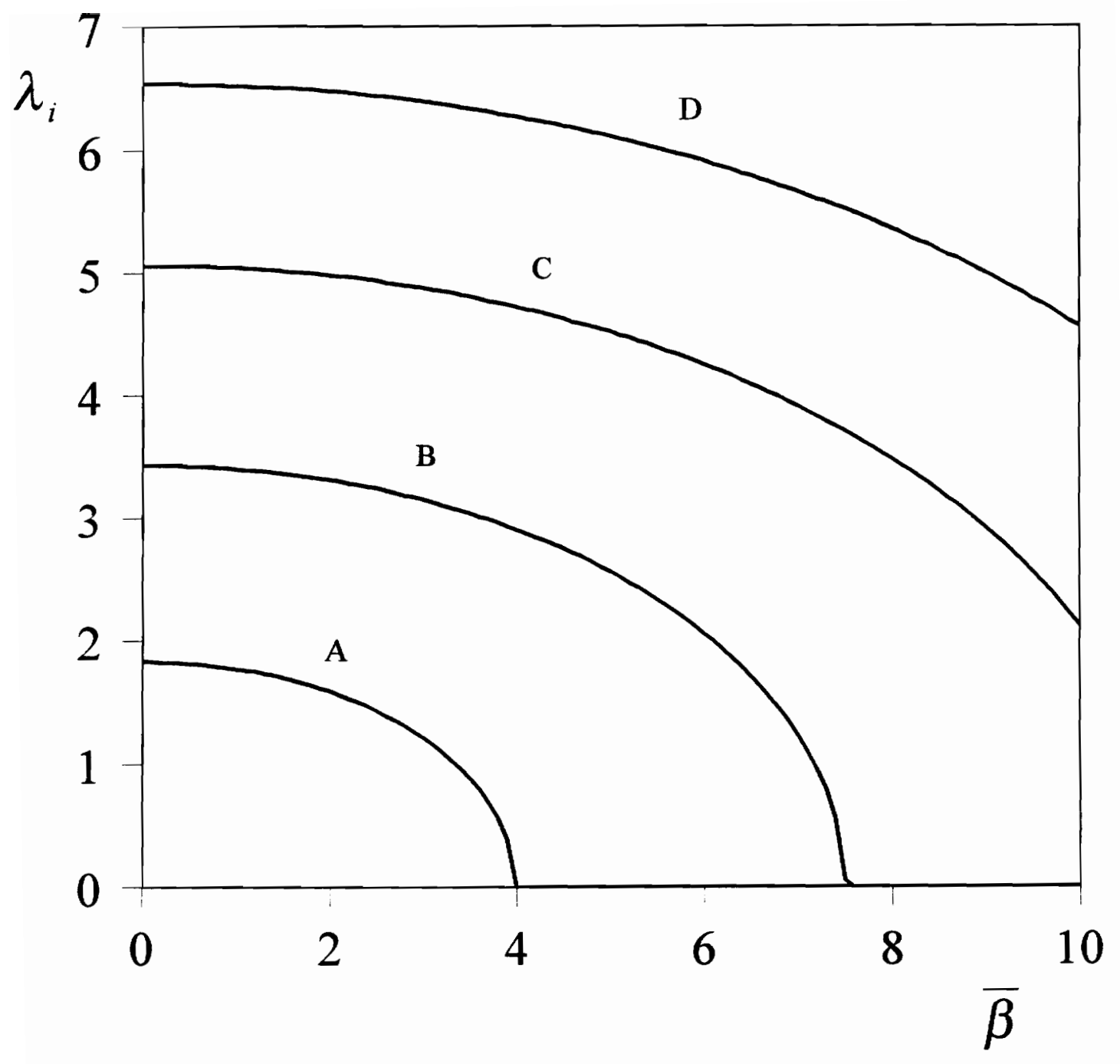

Figure 4.30 Imaginary Parts of Eigenvalues with $\bar{\beta}$ as $\bar{\rho}=1$ 


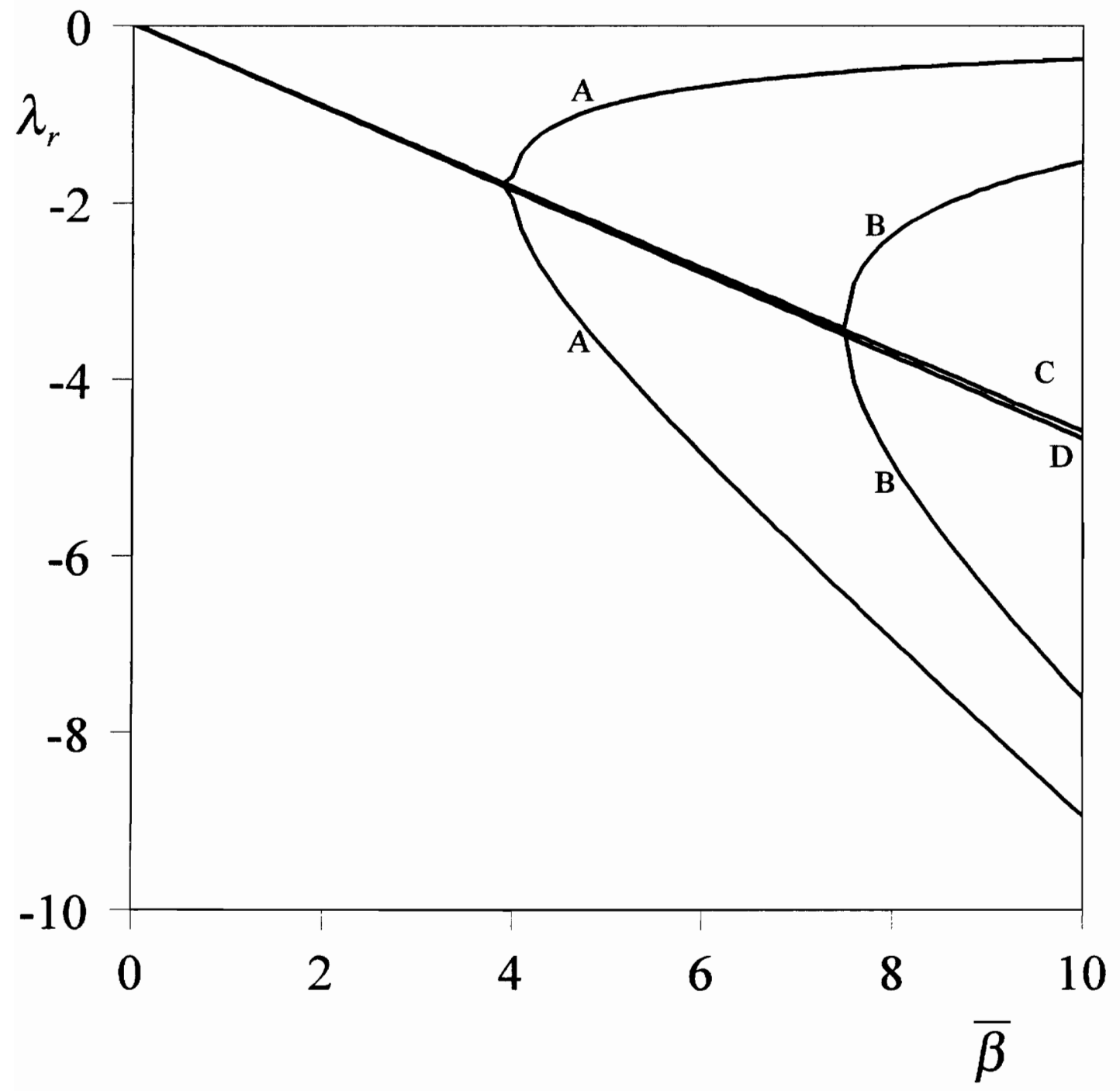

Figure 4.31 Real Parts of Eigenvalues with $\bar{\beta}$ as $\bar{\rho}=1$ 


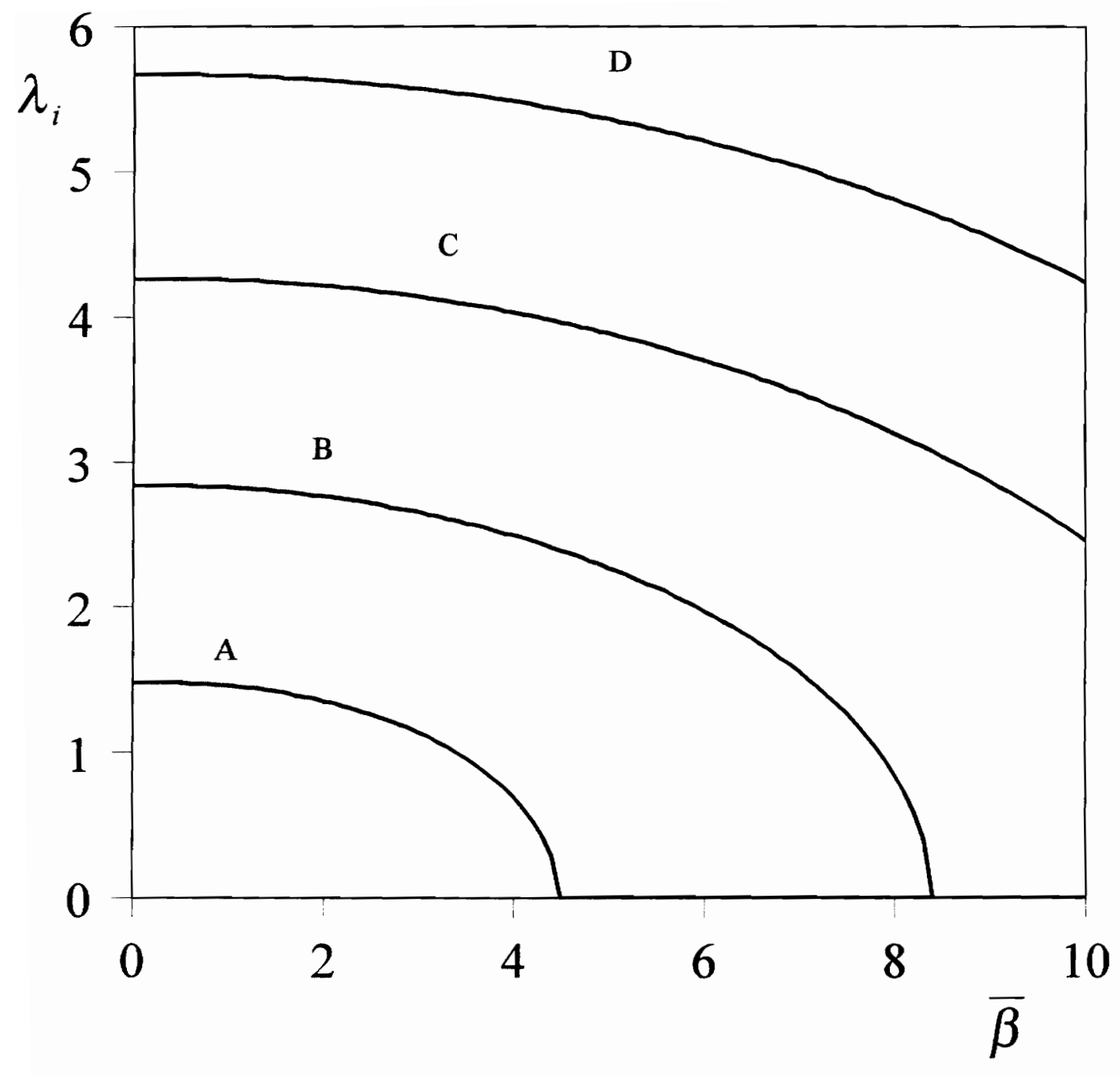

Figure 4.32 Imaginary Parts of Eigenvalues with $\bar{\beta}$ as $\bar{\rho}=5$ 


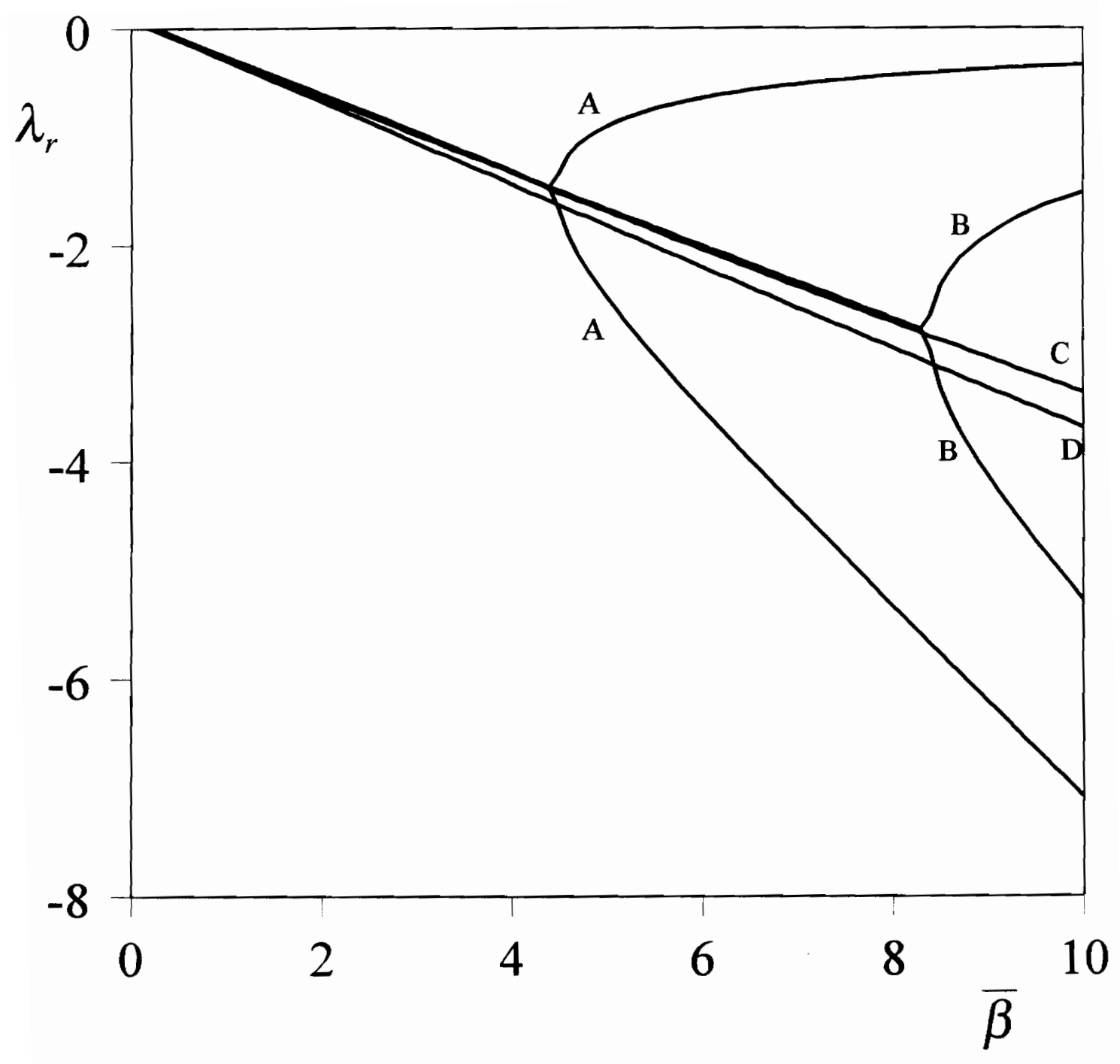

Figure 4.33 Real Parts of Eigenvalues with $\bar{\beta}$ as $\bar{\rho}=5$ 


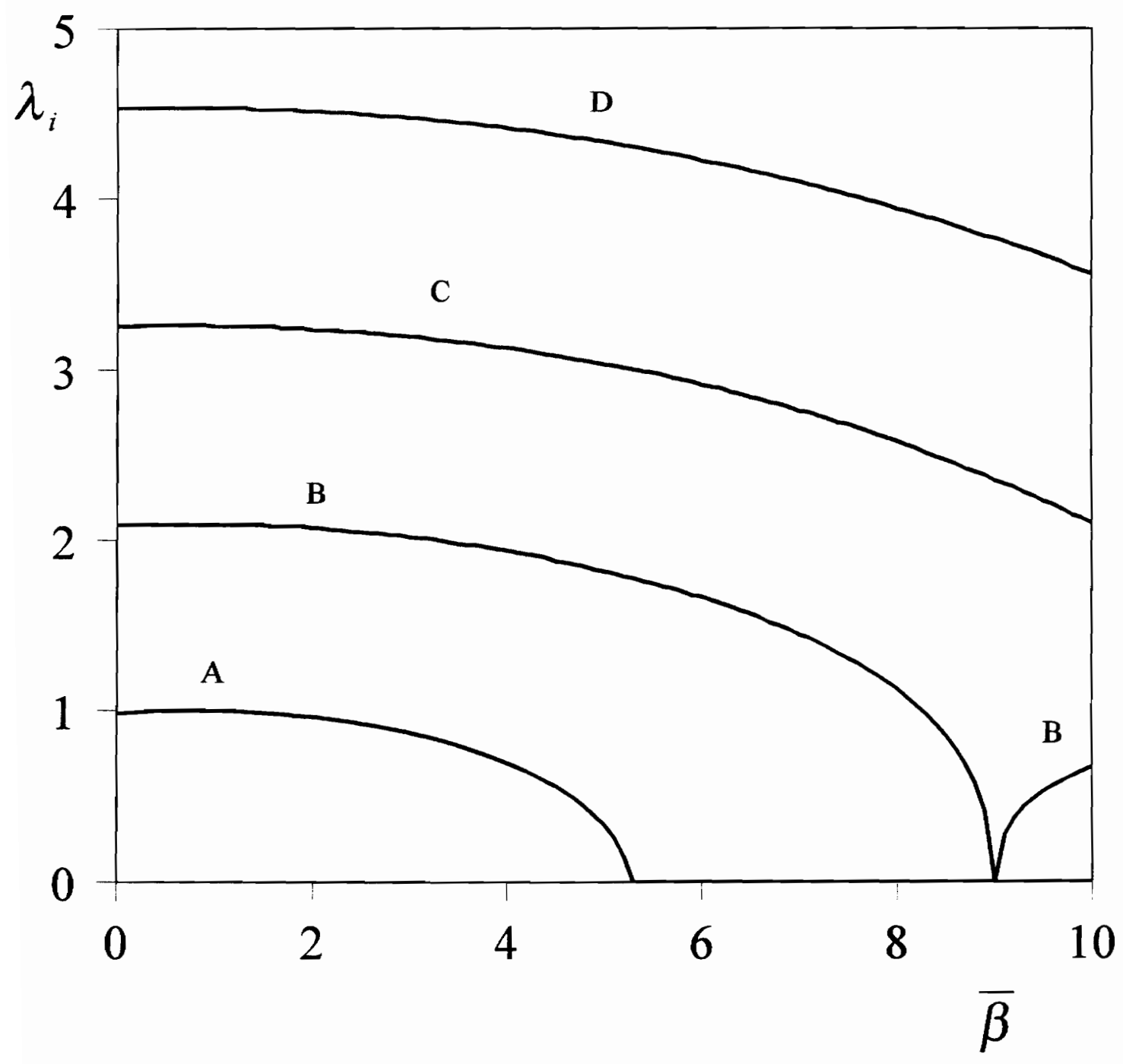

Figure 4.34 Imaginary Parts of Eigenvalues with $\bar{\beta}$ as $\bar{\rho}=12$ 

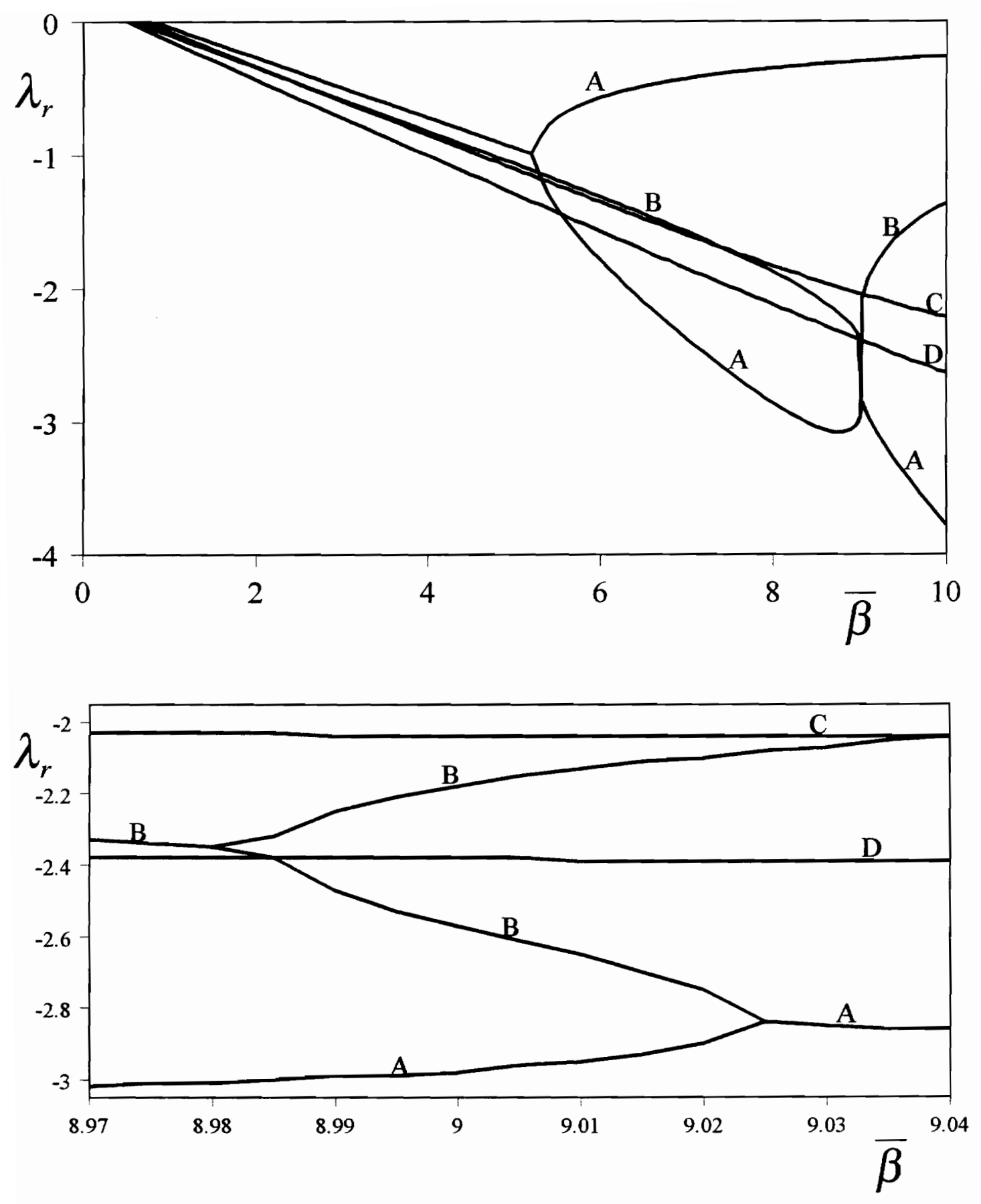

Figure 4.35 Real Parts of Eigenvalues with $\bar{\beta}$ as $\bar{\rho}=12$ 


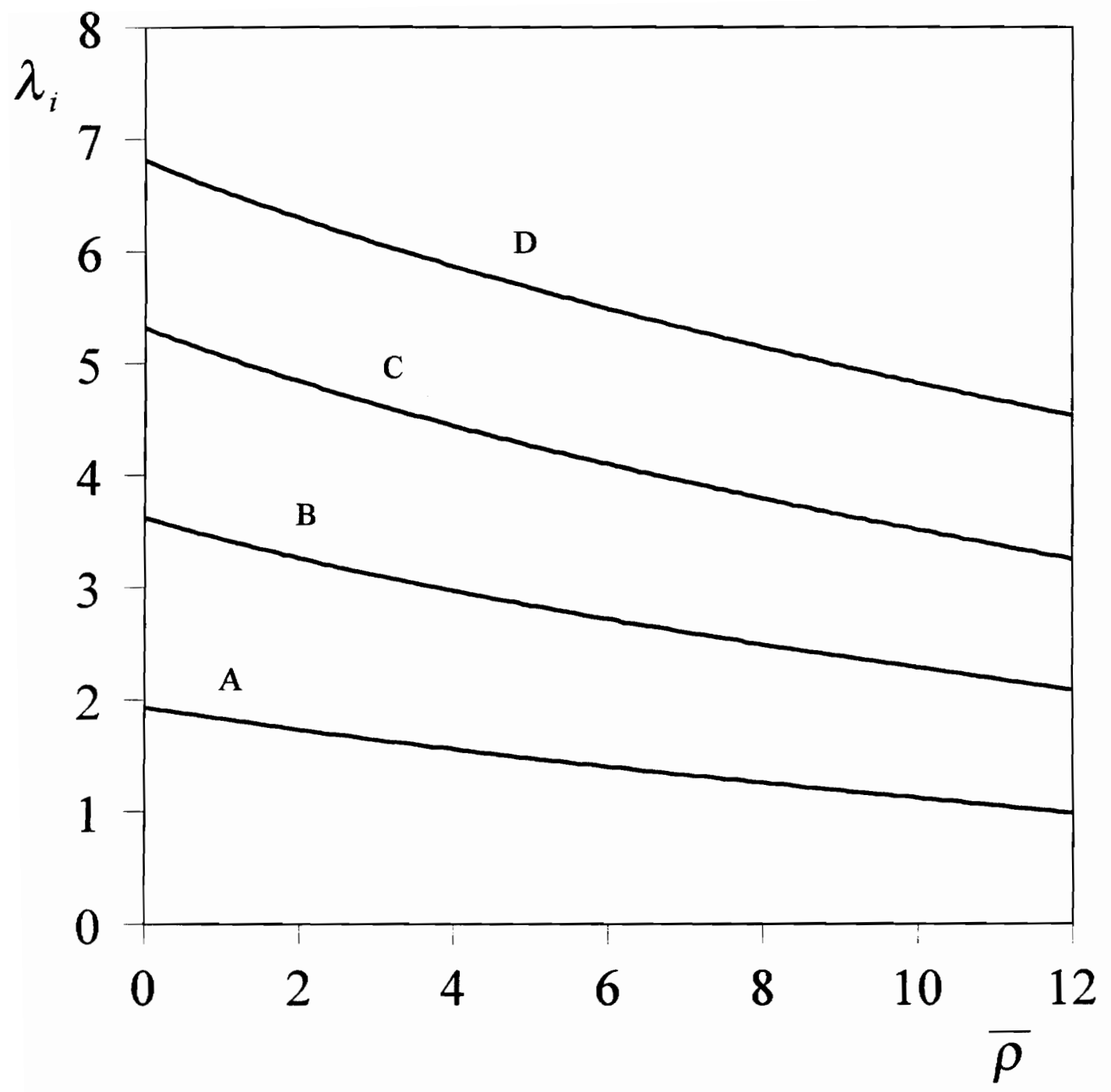

Figure 4.36 Imaginary Parts of Eigenvalues with $\bar{\rho}$ as $\bar{\beta}=0.1$ 


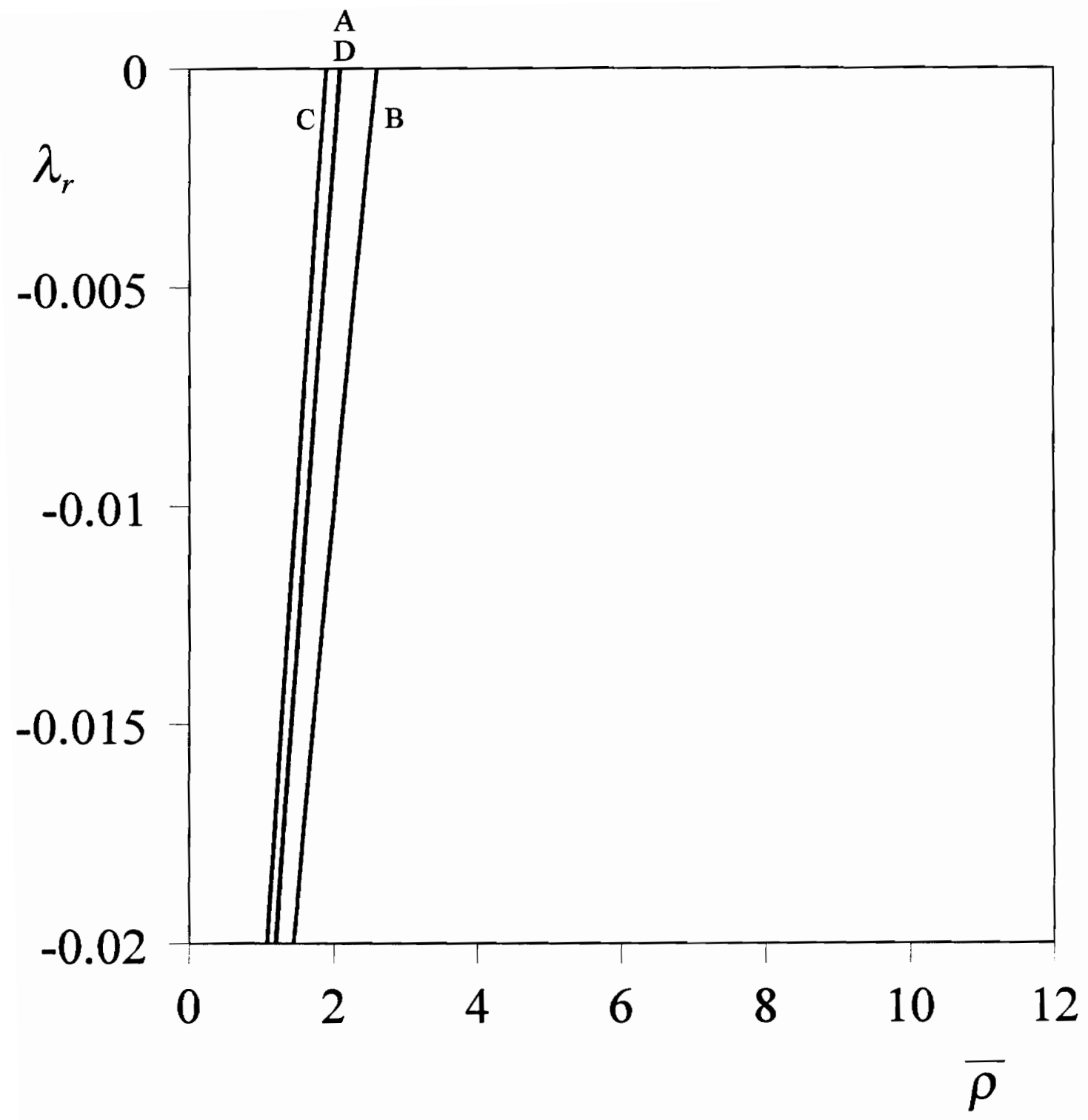

Figure 4.37 Real Parts of Eigenvalues with $\bar{\rho}$ as $\bar{\beta}=0.1$ 


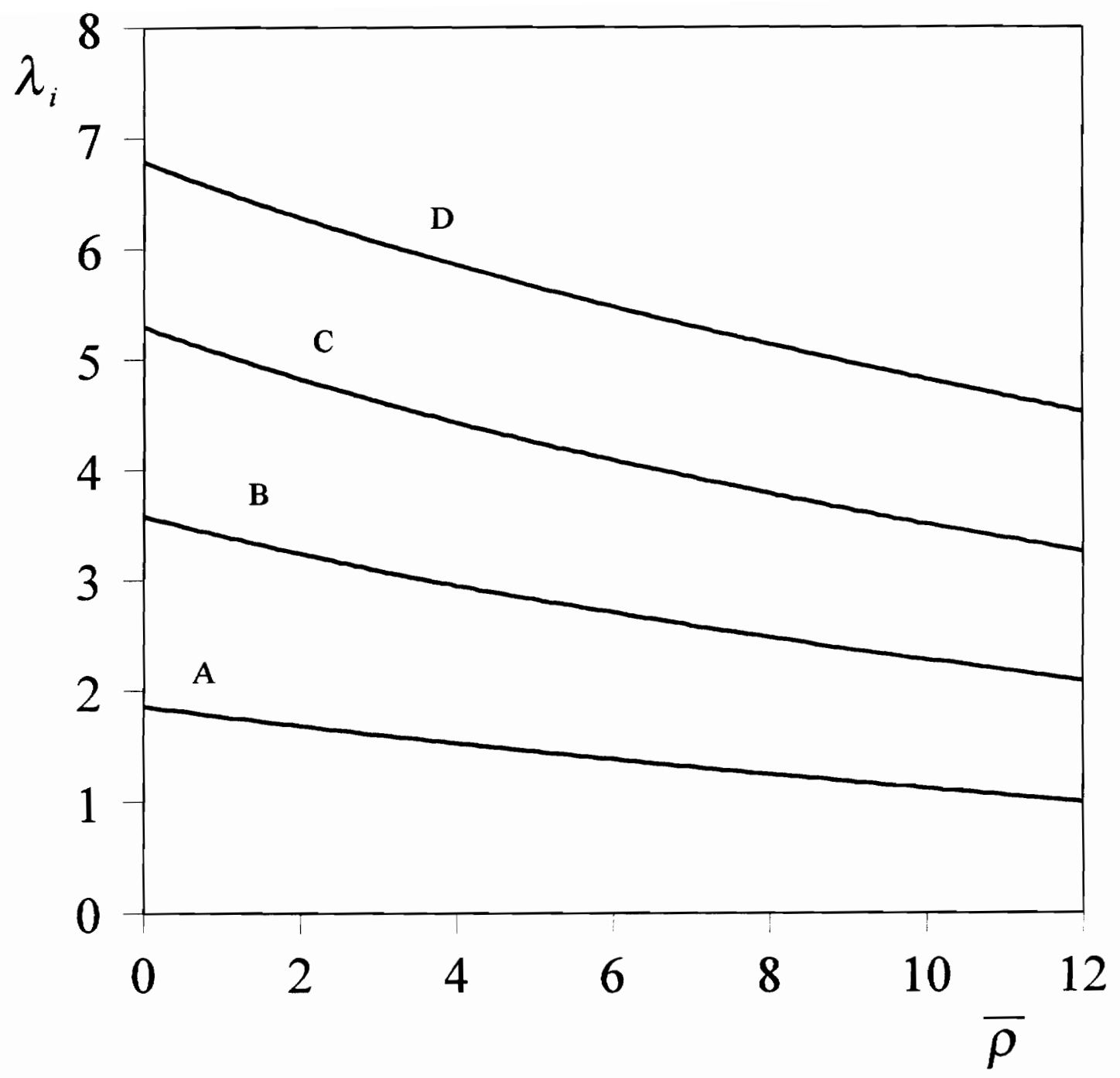

Figure 4.38 Imaginary Parts of Eigenvalues with $\bar{\rho}$ as $\bar{\beta}=1$ 


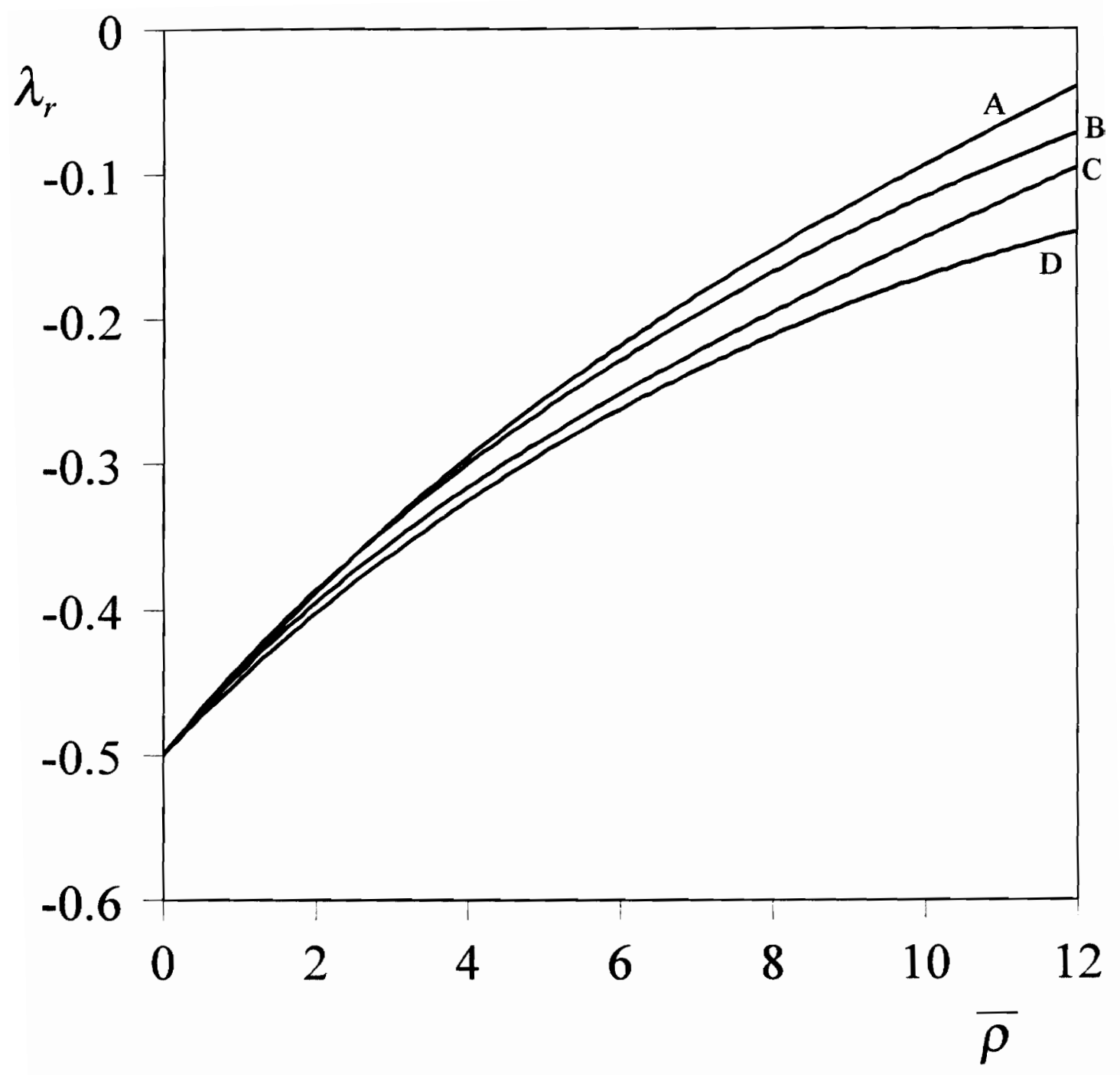

Figure 4.39 Real Parts of Eigenvalues with $\bar{\rho}$ as $\bar{\beta}=1$ 


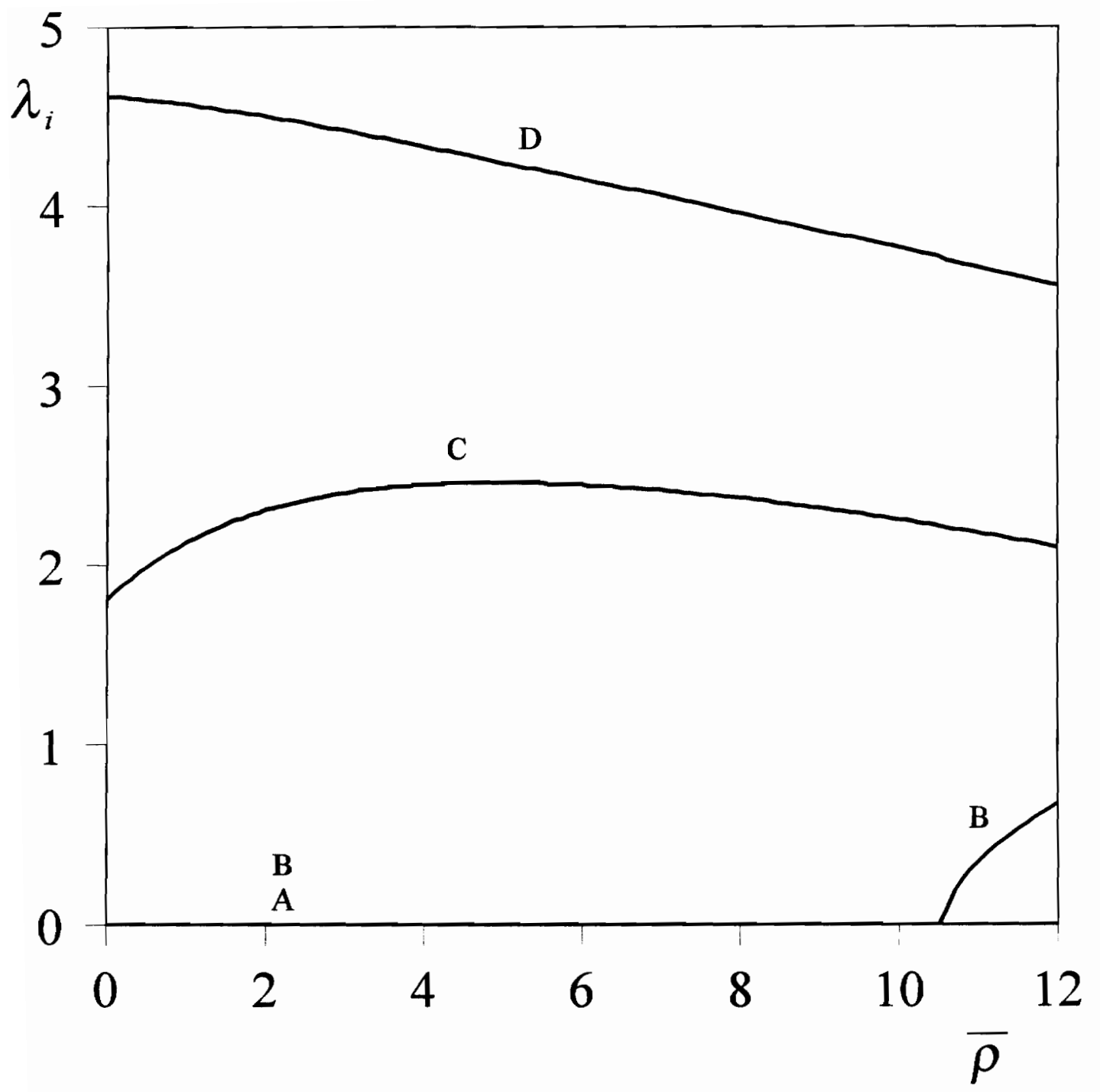

Figure 4.40 Imaginary Parts of Eigenvalues with $\bar{\rho}$ as $\bar{\beta}=10$ 


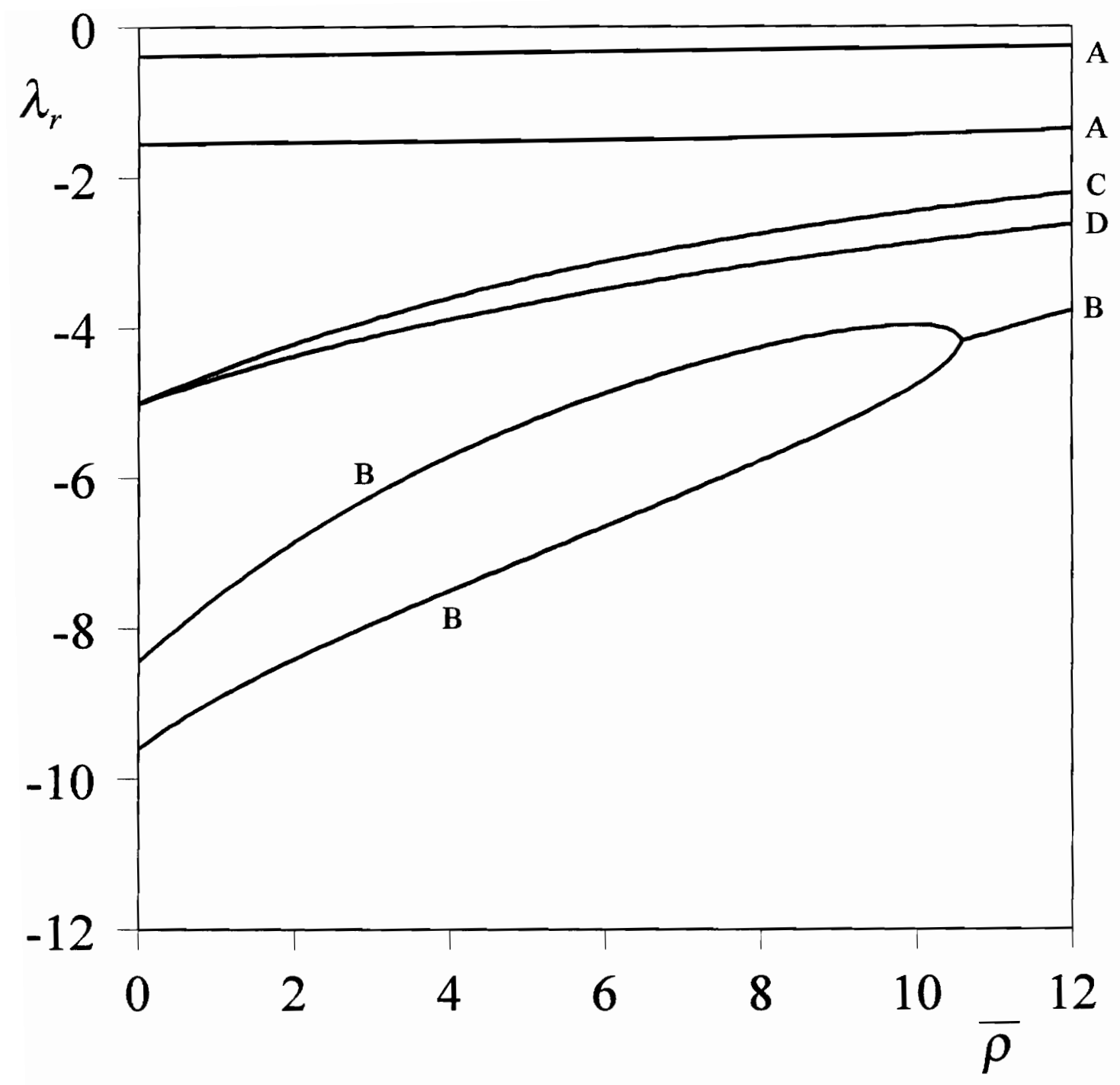

Figure 4.41 Real Parts of Eigenvalues with $\bar{\rho}$ as $\bar{\beta}=10$ 


\section{Chapter 5}

\section{CONCLUSIONS AND SUGGESTIONS}

\subsection{CONCLUSIONS}

The analysis of the vibrations of inflatable dams under overflow conditions is performed in this dissertation. The base width, curved perimeter, and internal air pressure of the dam are given, as well as the total head of the overflow.

The static analysis makes use of the alternating iterative approach which combines the boundary element method for the flow domain and the multiple shooting method for the shape of the dam. The profile of the free surface, the shape of the dam, and the external pressure distribution over the dam surface are obtained in the iterative scheme. The example in the static analysis displays good accuracy for the Bernoullivalues (total head) for the free surface.

Based on the result of the static analysis and with the use of the equations of motion for the membrane (in finite difference form) and the velocity potential formulation by the boundary element method, the eigenvalue problem is solved. Information on the eigenvalues and eigenvectors provides the description of the behavior of the dam, and the mode shapes for several examples are 
demonstrated. The effects of the water/dam density ratio and the damping coefficient parameter on the eigenvalues of the system are examined. These two parameters both involve the density of water, as well as the density of the dam (see the definitions of $\bar{\beta}$ in Equation (4.17) and $\bar{\rho}$ in Equation (4.42)). Under some conditions, the dam is unstable. Instability may be of the flutter type or the divergence type.

\subsection{SUGGESTIONS}

For the static problem, the free surface analysis is difficult. Possible improvements which can be made to the existing method include the free surface adjustment criteria, the initial guess of the free surface, the node setup, the scale-down factor for the change of the position, the curve-fitting between the subcritical and the supercritical flow regions, and the alternating iterative scheme. The other promising approaches for the free surface flow problem may be the inverse method (working on the stream function and the velocity potential domain instead of $\mathrm{x}$ and $\mathrm{y}$-coordinates), a combination of finite element and boundary element methods, and determination of the free surface from experiments.

In the dynamic analysis, we have the additional velocity potential caused by the membrane vibration equal to zero on the free surface, inflow boundary, and outflow boundary in this study. Other boundary conditions can be tried, e.g., the wave equation on the free surface. Taking account of the viscosity of the flow domain needs more effort, but it may be worthwhile.

The analysis of other forms of inflatable dams may give interesting results, such as the one with a fin beyond its crest which reduces the vibrations and the possibility of negative pressure on the downstream face of the dam. The fin generates separation and therefore introduces additional difficulty to the analysis of the overflow problem. There is also a type of dam with only a single fixed line along its longitudinal direction, which is more like a balloon, and an analysis of this type needs different assumptions and formulation. 


\section{REFERENCES}

Alwan, A. D., 1986, "Modal Analysis of Inflatable Dams under Hydrodynamic Conditions," Proceedings of the 4th International Modal Analysis Conference, Los Angeles, California, Vol. II, pp. 1502-1509.

Alwan, A. D., 1988, "Modal Analysis of Flexible Structure Used for Flood Control," Proceedings of the 6th International Modal Analysis Conference, Kissimmee, Florida, Vol. II, pp. $1180-1185$.

Anwar, H. O., 1967, "Inflatable Dams," Journal of the Hydraulics Division, ASCE, Vol. 93, No. HY3, pp. 99-119.

Bettess, P., and Bettess, J. A., 1983, "Analysis of Free Surface Flows using Isoparametric Finite Elements," International Journal for Numerical Methods in Engineering, Vol. 19, pp. 1675-1689.

Betts, P. L., 1979, "A Variational Principle in Terms of Stream Function for Free-Surface Flows and its Application to the Finite Element Method ," Computers and Fluids, Vol. 7, pp. 145-153.

Binnie, A. M., 1973, "The Theory of Flexible Dams Inflated by Water Pressure," Journal of Hydraulic Research, Vol. 11, pp. 61-68.

Binnie, G. M., Thomas, A. R., and Gwyther, J. R., 1974, "Inflatable Weir Used during Construction of Mangla Dam," Proceedings of the Institution of Civil Engineers, Part 1: Design and Construction, Vol. 54, pp. 625-639; Discussion by C. van Beesten, Vol. 56, 1974, pp. 189-191.

Bisplinghoff, R. L., and Ashley, H., 1962, Principles of Aeroelasticity, John Wiley, New York.

Bridgestone Engineered Products Co., 1991, Questions and Answers about the Bridgestone Rubber Dam, Tennessee, USA.

Cassidy, J. J., 1965, "Irrotational Flow over Spillways of Finite Height ," Journal of the Engineering Mechanics Division, ASCE, Vol. 91, No. EM6, pp. 155-173. 
Castro-Delgado, M., and Celik, I., 1986, "Analysis of Free-Surface Flows past Overflow Gates using Finite Element Method," Computers and Fluids, Vol. 14, No. 2, pp. 159-169.

Chan, S. T. K., Larock, B. E., and Herrmann, L. R., 1973, "Free-Surface Ideal Fluid Flows by Finite Elements," Journal of the Hydraulics Division, ASCE, Vol. 99, pp. 959-974.

Cheng, A. H-D., Liggett, J. A., and Liu, P. L-F., 1981, "Boundary Calculations of Sluice and Spillway Flows," Journal of the Hydraulics Division, ASCE, Vol. 107, pp. 1163-1178.

Chow, V. T., 1959, Open-Channel Hydraulics, McGraw-Hill Inc., New York.

Fagan, T. D., 1987, Effect of Membrane Weight on Vibrations of Air- Inflated Dams, M.S. Thesis, Virginia Polytechnic Institute and State University, Blacksburg, Virginia.

Firt, V., 1983, Statics, Formfinding and Dynamics of Air-Supported Membrane Structures, Martinus Nijhoff Publishers, The Hague, The Netherlands.

Forbes, L. K., 1988, "Critical Free-Surface Flow over a Semi-Circular Obstruction," Journal of Engineering Mathematics, Vol. 22, pp. 3-13.

Forbes, L. K., and Schwartz, L. W., 1982, "Free-Surface Flow over a Semicircular Obstruction," Journal of Fluid Mechanics, Vol. 114, pp. 299-314.

Harrison, H. B., 1970, "The Analysis and Behavior of Inflatable Membrane Dams under Static Loading," Proceedings of the Institution of Civil Engineers, Vol. 45, pp. 661-676.

Henrych, J., 1981, The Dynamics of Arches and Frames, Elsevier Scientific Publishing Company, Amsterdam.

Hsieh, J.-C., 1988, "Free Vibration of Inflatable Dams," Ph.D. Dissertation, Virginia Polytechnic Institute and State University, Blacksburg, Virginia.

Hsieh, J.-C., and Plaut, R. H., 1990, "Free Vibrations of Inflatable Dams," Acta Mechanica, Vol. 85 , pp. $207-220$.

Hsieh, J.-C., Plaut, R. H., and Yucel, O., 1989, "Vibrations of an Inextensible Cylindrical Membrane Inflated with Liquid," Journal of Fluids and Structures, Vol. 3, pp. 151-163.

Ikegawa, M., and Washizu, K., 1973, "Finite Element Method Applied to Analysis of Flow over a Spillway Crest," International Journal for Numerical Methods in Engineering, Vol. 6, pp. 179-189.

IMSL Math/Library, Fortran Subroutines for Mathematical Applications, 1989, Version 1.1, Houston, Texas.

Jovanovic, M. B., 1987, "Application of the Boundary Element Method for the Solution of Spillway Flows," Boundary Element Techniques: Applications in Fluid Flow and Computational Aspects, Editors: Brebbia, C. A., and Venturini, W. S., Computational Mechanics Publications, Southampton, U. K., pp. 101-109.

Leeuwrik, M. J., 1987, Nonlinear Vibration Analysis of Inflatable Dams, M.S. Thesis, Virginia Polytechnic Institute and State University, Blacksburg, Virginia.

Li, W., Xie, Q., and Chen, C. J., 1989, "Finite Analytic Solution of Flow over Spillways," Journal of Engineering Mechanics, Vol. 115, No. 12, pp. 2635-2648. 
Liggett, J. A., and Liu, P. L.-F., 1983, The Boundary Integral Equation Method for Porous Media Flow, George Allen \& Unwin Publishers, London.

Luke, J. C., 1967, "A Variational Principle for a Fluid with a Free Surface," Journal of Fluid Mechanics, Vol. 27, pp. 395-397.

Mathews, J. H., 1987, Numerical Methods for Computer Science, Engineering, and Mathematics, Prentice-Hall Inc., Englewood Cliffs, New Jersey.

Ogiwara, K., Tateishi, T., and Furuya H., 1970, "The Shape of the Rubber Dam," Proceedings, Japan Society of Civil Engineers, Vol. 179, No. 7, pp. 37-47.

Parbery, R. D., 1976, "A Continuous Method of Analysis for the Inflatable Dam," Proceedings of the Institution of Civil Engineers, Part 2, Vol. 61, pp. 725-736.

Parbery, R. D., 1978, "Factors Affecting the Membrane Dam Inflated by Air Pressure," Proceedings of the Institution of Civil Engineers, Part 2, Vol. 65, pp. 645-654.

Plaut, R. H., and Fagan, T. D., 1988, "Vibrations of an Inextensible, Air-Inflated, Cylindrical Membrane," Journal of Applied Mechanics, Vol. 55, pp. 672-675.

Plaut, R. H., and Leeuwrik, M. J., 1988, "Non-Linear Oscillations of an Inextensible, Air-Inflated, Cylindrical Membrane," International Journal of Non-Linear Mechanics, Vol. 23, pp. 347-353.

Sabersky, R. H., Acosta, A. J., and Hauptmann, E. G., 1989, Fluid Flow, Macmillan Publishing Company, New York.

Southwell, R. V., and Vaisey, G., 1946, "Relaxation Methods Applied to Engineering Problems: XII, Fluid Motions Characterized by Free Streamlines," Philosophical Transactions, Royal Society of London, Series A, Vol. 240, England, pp. 117-161.

University of Sidney, Civil Engineering Laboratories, 1969, "Report of an Investigation into the Failure of an Inflatable Dam," Investigation Report No. S89, May.

Varoglu, E., and Finn, W. D. L., 1978, "Variable Domain Finite Element Analysis of Free Surface Gravity Flow," Computers and Fluids, Vol. 6, pp. 103-114.

von Karman, T., 1940, "The Engineer Grapples with Nonlinear Problems," Bulletin of the American Mathematical Society, Vol. 46, pp. 615-683.

Watson, R., 1985, "A Note on the Shapes of Flexible Dams," Journal of Hydraulic Research, Vol. 23, pp. 179-194. 


\section{Appendix A}

\section{THE FINITE DIFFERENCE SCHEME}

The finite difference scheme developed here is utilized to solve the equation of motion for the membrane (Equation (4.23)), the boundary condition at the dam-flow interface (Equation (4.55)), and the Bernoulli equation at the dam-flow interface (Equation (4.66)). A uniform interval, $\bar{h}$, specifies the curved spacing length between neighboring nodes on the dam throughout the formulation in which central differences, forward differences, backward differences, and their combinations are employed.

\section{A.1 FINITE DIFFERENCE FOR EQUATION (4.23)}

In Equation (4.23), the derivatives of $V_{i}, P_{i}$ and $\bar{\theta}_{0 i}$ (in the form of $\bar{b}_{k i}, k=1,2, \ldots, 8$; see Equations (4.30), (4.17), (4.16), and (4.12)), $(i=1,2, \ldots, m)$ are to be put in finite difference form. For the derivatives of $V_{i}$, the following central differences of order $O\left(\bar{h}^{2}\right)$ are used (e.g., see Mathews, 1987): 


$$
\begin{aligned}
& V_{i}^{\prime}=\frac{1}{2 \bar{h}}\left(V_{i+1}-V_{i-1}\right) ; \quad V_{i}^{\prime \prime}=\frac{1}{\bar{h}^{2}}\left(V_{i+1}-2 V_{i}+V_{i-1}\right) ; \\
& V_{i}^{\prime \prime \prime}=\frac{1}{2 \bar{h}^{3}}\left(V_{i+2}-2 V_{i+1}+2 V_{i-1}-V_{i-2}\right) ; \\
& V_{i}^{\prime \prime \prime \prime}=\frac{1}{\bar{h}^{4}}\left(V_{i+2}-4 V_{i+1}+6 V_{i}-4 V_{i-1}+V_{i-2}\right)
\end{aligned}
$$

$$
(i=1,2, \ldots, m)
$$

where the terms $V_{0}, V_{-1}, V_{m+1}$ and $V_{m+2}$ can be replaced as follows with the help of the boundary conditions (Equation (4.25)):

$$
\begin{aligned}
& V_{1}^{\prime}=\frac{1}{2 \bar{h}} V_{2} ; \quad V_{m}^{\prime}=-\frac{1}{2 \bar{h}} V_{m-1} ; \\
& V_{1}^{\prime \prime}=\frac{1}{\bar{h}^{2}}\left(V_{2}-2 V_{1}\right) ; \quad V_{m}^{\prime \prime}=\frac{1}{\bar{h}^{2}}\left(-2 V_{m}+V_{m-1}\right) ; \\
& V_{1}^{\prime \prime \prime}=\frac{1}{2 \bar{h}^{3}}\left(V_{3}-2 V_{2}-V_{1}\right) ; \quad V_{2}^{\prime \prime \prime}=\frac{1}{2 \bar{h}^{3}}\left(V_{4}-2 V_{3}+2 V_{1}\right) ; \\
& V_{m}^{\prime \prime \prime}=\frac{1}{2 \bar{h}^{3}}\left(V_{m}+2 V_{m-1}-V_{m-2}\right) ; \quad V^{\prime \prime \prime} m-1=\frac{1}{2 \bar{h}^{3}}\left(-2 V_{m}+2 V_{m-2}-V_{m-3}\right) ; \\
& V_{1}^{\prime \prime \prime \prime}=\frac{1}{\bar{h}^{4}}\left(V_{3}-4 V_{2}+7 V_{1}\right) ; \quad V_{2}^{\prime \prime \prime \prime}=\frac{1}{\bar{h}^{4}}\left(V_{4}-4 V_{3}+6 V_{2}-4 V_{1}\right) ; \\
& V_{m}^{\prime \prime \prime \prime}=\frac{1}{\bar{h}^{4}}\left(7 V_{m}-4 V_{m-1}+V_{m-2}\right) ; \\
& V_{m-1}^{\prime \prime \prime \prime}=\frac{1}{\bar{h}^{4}}\left(-4 V_{m}+6 V_{m-1}-4 V_{m-2}+V_{m-3}\right)
\end{aligned}
$$

The central differences of order $O\left(\bar{h}^{2}\right)$ are used for the first derivative of $P_{i}$ as well:

$$
P_{i}^{\prime}=\frac{1}{2 \bar{h}}\left(P_{i+1}-P_{i-1}\right)
$$




$$
(i=1,2, \ldots, m)
$$

For the derivatives of $\bar{\theta}_{0 i}$, first we apply the central differences of order $O\left(\bar{h}^{2}\right)$ to Equation (4.12) with the help of the nondimensional forms in Equation (4.17):

$$
\bar{a}_{1 i}=\frac{1}{2 \bar{h}}\left(\bar{\theta}_{0 ; i+1}-\bar{\theta}_{0 ; i-1}\right) ; \quad \bar{a}_{2 i}=\frac{1}{\bar{h}^{2}}\left(\bar{\theta}_{0 ; i+1}-2 \bar{\theta}_{0 i}+\bar{\theta}_{0 ; i-1}\right)
$$

$$
(i=1,2, \ldots, m)
$$

$$
\begin{aligned}
& \bar{a}_{3 i}=\frac{1}{2 \bar{h}^{3}}\left(\bar{\theta}_{0 ; i+2}-2 \bar{\theta}_{0 ; i+1}+2 \bar{\theta}_{0 ; i-1}-\bar{\theta}_{0 ; i-2}\right) \\
& \bar{a}_{4 i}=\frac{1}{\bar{h}^{4}}\left(\bar{\theta}_{0 ; i+2}-4 \bar{\theta}_{0 ; i+1}+6 \bar{\theta}_{0 i}-4 \bar{\theta}_{0 ; i-1}+\bar{\theta}_{0 ; i-2}\right)
\end{aligned}
$$

$$
(i=2,3, \ldots, m-1)
$$

Then a combination of forward differences of order $O(\bar{h})$ and central differences of order $O\left(\bar{h}^{2}\right)$ is used on $\bar{a}_{31}$ and $\bar{a}_{41}$ :

$$
\begin{aligned}
& \bar{a}_{31}=\frac{1}{\bar{h}}\left(\bar{a}_{22}-\bar{a}_{21}\right)=\frac{1}{\bar{h}^{3}}\left(\bar{\theta}_{03}-3 \bar{\theta}_{02}+3 \bar{\theta}_{01}-\bar{\theta}_{00}\right) ; \\
& \bar{a}_{41}=\frac{1}{\bar{h}}\left(\bar{a}_{32}-\bar{a}_{31}\right)=\frac{1}{\bar{h}^{4}}\left(\frac{1}{2} \bar{\theta}_{04}-2 \bar{\theta}_{03}+3 \bar{\theta}_{02}-2 \bar{\theta}_{01}+\frac{1}{2} \bar{\theta}_{00}\right)
\end{aligned}
$$

The quantities $\bar{a}_{3 m}$ and $\bar{a}_{4 m}$ are expressed partly by backward differences of order $O(\bar{h})$ and partly by central differences of order $O\left(\bar{h}^{2}\right)$ :

$$
\begin{aligned}
& \bar{a}_{3 m}=\frac{1}{\bar{h}}\left(\bar{a}_{2 m}-\bar{a}_{2 ; m-1}\right)=\frac{1}{\bar{h}^{3}}\left(\bar{\theta}_{0 ; m+1}-3 \bar{\theta}_{0 m}+3 \bar{\theta}_{0 ; m-1}-\bar{\theta}_{0 ; m-2}\right) ; \\
& \bar{a}_{4 m}=\frac{1}{\bar{h}}\left(\bar{a}_{3 m}-\bar{a}_{3 ; m-1}\right) \\
& =\frac{1}{\bar{h}^{4}}\left(\frac{1}{2} \bar{\theta}_{0 ; m+1}-2 \bar{\theta}_{0 m}+3 \bar{\theta}_{0 ; m-1}-2 \bar{\theta}_{0 ; m-2}+\frac{1}{2} \bar{\theta}_{0 ; m-3}\right)
\end{aligned}
$$


Equations (A.4)-(A.7), and Equation (4.30) which provides the relations between $\bar{a}_{k j}$ and $\bar{b}_{i j}$ $(k=1,2,3,4 ; i=1,2, \ldots, 8 ; j=1,2, \ldots, m)$, give $\bar{b}_{i j}$ in Equation (4.23). Moreover, the derivatives of $V_{i}$ and $P_{i}$ are replaced with Equations (A.1)-(A.3) to obtain Equation (4.26).

\section{A.2 FINITE DIFFERENCE FOR EQUATION (4.55)}

In Equation (4.55), $V_{j}^{\prime}, V_{j}^{\prime \prime}$ and $\bar{a}_{1 j}, \bar{a}_{2 j}(j=1,2, \ldots, m)$ are the same as those in Equations (A.1), (A.2) and (A.4); however, the terms defined at the fixed ends ( $j=0$ and $j=m+1)$ need different formulations. For the derivatives of $V_{0}$ and $V_{m+1}$,

$$
\begin{aligned}
& V_{0}^{\prime}=\frac{1}{\bar{h}}\left(V_{1}-V_{0}\right)=\frac{1}{\bar{h}} V_{1} ; \\
& V_{m+1}^{\prime}=\frac{1}{\bar{h}}\left(V_{m+1}-V_{m}\right)=-\frac{1}{\bar{h}} V_{m}
\end{aligned}
$$

where the forward and backward differences of order $O(\bar{h})$ and the boundary conditions (Equation (4.25)) are used;

$$
\begin{aligned}
& V_{0}^{\prime \prime}=\frac{1}{\bar{h}^{2}}\left(V_{1}-2 V_{0}+V_{-1}\right)=\frac{1}{\bar{h}^{2}}\left(2 V_{1}\right) \\
& V_{m+1}^{\prime \prime}=\frac{1}{\bar{h}^{2}}\left(V_{m+2}-2 V_{m+1}+V_{m}\right)=\frac{1}{\bar{h}^{2}}\left(2 V_{m}\right)
\end{aligned}
$$

where the central differences of order $O\left(\bar{h}^{2}\right)$ and the boundary conditions (Equation (4.25)) are used.

For $\bar{a}_{1 ; 0}, \bar{a}_{1 ; m+1}, \bar{a}_{2 ; 0}$, and $\bar{a}_{2 ; m+1}$, we have

$$
\begin{aligned}
& \bar{a}_{1 ; 0}=\frac{1}{\bar{h}}\left(\bar{\theta}_{01}-\bar{\theta}_{00}\right) ; \quad \bar{a}_{1 ; m+1}=\frac{1}{\bar{h}}\left(\bar{\theta}_{0 ; m+1}-\bar{\theta}_{0 m}\right) ; \\
& \bar{a}_{2 ; 0}=\frac{1}{\bar{h}}\left(\bar{a}_{11}-\bar{a}_{10}\right)=\frac{1}{2 \bar{h}^{2}}\left(\bar{\theta}_{02}-2 \bar{\theta}_{01}+\bar{\theta}_{00}\right) ;
\end{aligned}
$$


$\bar{a}_{2 ; m+1}=\frac{1}{\bar{h}}\left(\bar{a}_{1 ; m+1}-\bar{a}_{1 m}\right)=\frac{1}{2 \bar{h}^{2}}\left(\bar{\theta}_{0 ; m+1}-2 \bar{\theta}_{0 m}+\bar{\theta}_{0 ; m-1}\right)$

where the forward and backward differences of order $O(\bar{h})$ are used and the central differences of order $O\left(\bar{h}^{2}\right)$ for $\bar{a}_{11}$ and $\bar{a}_{1 m}$ in Equation (A.4) are recalled for $\bar{a}_{2 ; 0}$ and $\bar{a}_{2 ; m+1}$.

The terms $\bar{\phi}_{0 j, s}(j=0,1, \ldots, m+1)$ are expressed by the forward differences of order $O(\bar{h})$ except that $\bar{\phi}_{0 ; m+1, \bar{s}}$ takes the backward difference of the same order:

$\bar{\phi}_{0 j, \bar{s}}=\frac{1}{\bar{h}}\left(\bar{\phi}_{0 ; j+1}-\bar{\phi}_{0 j}\right)$

$(j=0,1, \ldots, m)$

$\bar{\phi}_{0 ; m+1, \bar{s}}=\frac{1}{\bar{h}}\left(\bar{\phi}_{0 ; m+1}-\bar{\phi}_{0 m}\right)$

From the finite differences in this section, the coefficients $M_{j+1 ; k}$ and $N_{j+1 ; k}$ for $V_{k}$ in Equation (4.55) can be written as follows:

$M_{j+1 ; j-1}=-\frac{\bar{\phi}_{0 j, \bar{s}}}{\bar{h}^{2} \bar{a}_{1 j}}-\frac{\bar{\phi}_{0 j, \bar{s}} \bar{a}_{2 j}}{2 \bar{h} \bar{a}_{1 j}^{2}} ; \quad N_{j+1 ; j-1}=\frac{1}{2 \bar{h} \bar{a}_{1 j}}$

$M_{j+1 ; j}=\frac{2 \bar{\phi}_{0 j, \bar{s}}}{\bar{h}^{2} \bar{a}_{1 j}} ; \quad N_{j+1 ; j}=0$

$(j=1,2, \ldots, m)$

$M_{j+1 ; j+1}=-\frac{\bar{\phi}_{0 j, \bar{s}}}{\bar{h}^{2} \bar{a}_{1 j}}+\frac{\bar{\phi}_{0 j, \bar{s}} \bar{a}_{2 j}}{2 \bar{h} \bar{a}_{1 j}^{2}} ; \quad N_{j+1 ; j+1}=-\frac{1}{2 \bar{h} \bar{a}_{1 j}}$

$(j=1,2, \ldots, m-1)$

$M_{11}=-\frac{2 \bar{\phi}_{00, \bar{s}}}{\bar{h}^{2} \bar{a}_{1 ; 0}}+\frac{2 \bar{\phi}_{00, \bar{s}} \bar{a}_{2 ; 0}}{\bar{h} \bar{a}_{1 ; 0}^{2}} ; \quad N_{11}=-\frac{2}{\bar{h} \bar{a}_{1 ; 0}}$

$M_{12}=-\frac{\bar{\phi}_{00, \bar{s}} \bar{a}_{2 ; 0}}{2 \bar{h} \bar{a}_{1 ; 0}^{2}} ; \quad N_{12}=\frac{1}{2 \bar{h} \bar{a}_{1 ; 0}}$ 


$$
\begin{aligned}
& M_{m+2 ; m+1}=\frac{\bar{\phi}_{0 ; m+1, s} \bar{a}_{2 ; m+1}}{2 \bar{h}_{1 ; m+1}^{2}} ; \quad N_{m+2 ; m+1}=-\frac{1}{2 \bar{h} \bar{a}_{1 ; m+1}} \\
& M_{m+2 ; m+2}=-\frac{2 \bar{\phi}_{0 ; m+1, \bar{s}}}{\bar{h}^{2} \bar{a}_{1 ; m+1}}-\frac{2 \bar{\phi}_{0 ; m+1, \bar{s}} \bar{a}_{2 ; m+1}}{\bar{h} \bar{a}_{1 ; m+1}^{2}} ; \quad N_{m+2 ; m+2}=\frac{2}{\bar{h} \bar{a}_{1 ; m+1}}
\end{aligned}
$$

Other $M_{j+1 ; k}=0 ; \quad$ Other $N_{j+1 ; k}=0$

where $\bar{\phi}_{0 j, \xi}$ is defined in Equation (A.11).

\section{A.3 FINITE DIFFERENCE FOR EQUATION (4.66)}

For Equation (4.66), $\bar{\phi}_{0 i, s}$ and $V_{i}^{\prime}$ are the same as those in Equations (A.11) and (A.1)-(A.2), (A.8), respectively. This gives $H_{i+1 ; j+1}$ in Equation (4.67):

$$
\begin{aligned}
& H_{i+1 ; i}=-\frac{\bar{\phi}_{0 i, \bar{s}}}{2 \bar{h}} ; \quad H_{i+1 ; i+2}=\frac{\bar{\phi}_{0 i, \bar{s}}}{2 \bar{h}} \\
& H_{11}=-\frac{\bar{\phi}_{00, \bar{s}}}{\bar{h}} ; \quad H_{12}=\frac{\bar{\phi}_{00, \bar{s}}}{\bar{h}} \\
& H_{m+2 ; m+1}=-\frac{\bar{\phi}_{0 ; m+1, \bar{s}} ; \quad H_{m+2 ; m+2}=\frac{\bar{\phi}_{0 ; m+1, \bar{s}}}{\bar{h}}}{\bar{h}}
\end{aligned}
$$

Other $H_{i+1 ; j+1}=0$

where $\bar{\phi}_{0 i, s}$ is defined in Equation (A.11). Also,

$$
\begin{array}{lr}
K_{i+1 ; i-1}=-\frac{\cos \bar{\theta}_{0 i}}{2 \bar{h} \bar{a}_{1 i}} & (i=2,3, \ldots, m) \\
K_{i+1 ; i}=\sin \bar{\theta}_{0 i} & (i=1,2, \ldots, m)
\end{array}
$$




$$
K_{i+1 ; i+1}=\frac{\cos \bar{\theta}_{0 i}}{2 \bar{h} \bar{a}_{1 i}}
$$

$$
(i=1,2, \ldots, m-1)
$$

Other $K_{i+1 ; j}=0$ 


\section{Vita}

The author was born July 6, 1963 in Taiwan, ROC. He graduated from National Chiao Tung University at Hsinchu in Taiwan, with a B.S. in Civil Engineering in 1985 and an M.S. at the structures division of the same department in 1987. Then he served in the army as an officer in the corps of engineers for two years and came to Virginia Polytechnic Institute and State University in 1990. Upon acquiring his Ph.D. degree, he will pursue his career as a structural engineer.

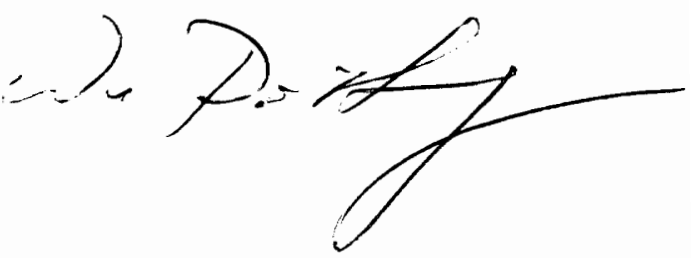

